\title{
Multimedidas vetoriais: extensão e integração
}

\section{Adriana Luiza do Prado}

\author{
DISSERTAÇÃO APRESENTADA \\ $\mathrm{AO}$ \\ INSTITUTO DE MATEMÁTICA E ESTATÍSTICA \\ DA \\ UNIVERSIDADE DE SÃO PAULO \\ PARA \\ OBTENÇÃO DO GRAU DE MESTRE \\ EM \\ MATEMÁTICA
}

Área de Concentração: Teoria da medida e Análise Funcional Orientadora: Profa. Dra. Carmen Silvia Cardassi

Durante a elaboraçào deste trabalho a autora recebeu apoio financeiro da CAPES

-São Paulo, dezembro de 1998- 


\section{MULTIMEDIDAS VETORIAIS: EXTENSÃO E INTEGRAÇÃO}

Este exemplar corresponde à redação da dissertação corrigida e defendida por Adriana Luiza do Prado e aprovada pela comissão julgadora.

São Paulo, 17 de dezembro de 1998.

\section{Comissão Julgadora}

- Profa. Dra. Carmen Sílvia Cardassi (Orientadora) - IME - USP

- Prof. Dr. Alfredo Jorge Aragona Vallejo - IME - USP

- Prof. Dr. Dicesar Lass Fernandez-IMECC-UNICAMP. 
À minha mãe D. Maria Luiza e aos meus amigos, por cada etapa 
A gente é o que é, (fazer o quê? ) mas também a gente é o que vier a ser ( então há tanto por fazer) 


\section{Agradecimentos}

Silvia

Agradeço a atenção, a disponibilidade, a paciência e a orientação da Professora Carmen

Agradeço a todas as pessoas que de uma maneira ou de outra contribuiram para este trabalho, meus sinceros agradecimentos. Peço desculpas aos que foram involutária e injustamente omitidos.

Quero agradecer especialmente à Adriana Junko (Elita), ao Valério, ao Fernando, colegas do IME, à Luciene e Rosa (colegas de morada) e tantos outros que seria impossível citá-los aqui. Também aos colegas do departamento de matemática da UFPR, em particular, ao Aurélio e à Marelin, professores e grandes incentivadores, ao Yuan e ao Trovon, pelas sugestões. 


\title{
Sumário
}

\author{
Agradecimentos
}

Introdução

1 Preliminares 4

1.1 Alguns resultados . . . . . . . . . . . . . . . . . 4

1.2 Funções convexas e funções semicontínuas inferiormente . . . . . . . . . . 6

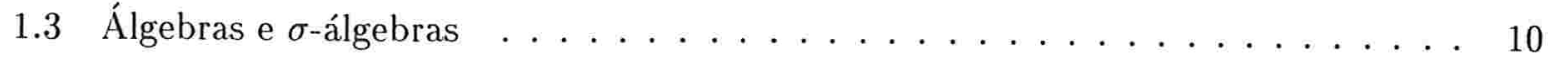

2 Medida e multimedida $\quad 13$

2.1 Medidas vetoriais e integração vetorial . . . . . . . . . . . . . . 13

2.2 Multimedidas . . . . . . . . . . . . . . . . . . 17

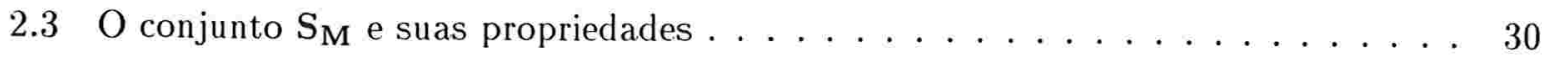

$3 \quad$ Extensão de uma multimedida $\quad 38$

4 Integração com respeito a uma multimedida $\quad 47$

$\begin{array}{ll}\text { Bibliografia } & 52\end{array}$ 


\begin{abstract}
Resumo
Este trabalho tem por objetivo estudar multimedidas definidas de álgebras em subconjuntos de um espaço de Banach real $X$. A primeira pergunta é como podemos estender multimedida de uma álgebra para uma multimedida numa $\sigma$-álgebra. A segunda pergunta é qual a relação entre a multimedida e medida vetorial. Mostramos aqui vários resultados apresentados por D.Kandilakis ([9]), assumindo no último caso que $X$ é reflexivo. Finalmente, nós estudamos as funções de conjuntos obtidas pela integração de uma função limitada mensurável com respeito a uma multimedida.
\end{abstract}

\begin{abstract}
The main purpose of this work is to study the multimeasure defined on a field in the subsets of the real Banach space $X$. The first question is how we can extend multimeasure on a field to one on a $\sigma$-field. The second one is what the relation between multimeasure and vectorial measure is. We show several results presented by D.Kandilakis ([9]), in the last case of which we assume that $X$ is reflexive. Finally, we study the set functions obtained by integrating a bounded measurable function with respect to a multimeasure.
\end{abstract}




\section{Introdução}

Esta dissertação trata de extensão e integração de multimedidas vetoriais. Foi feito um estudo com medidas vetoriais e como estender algumas das propriedades destas medidas para as multimedidas.

O estudo de multimedidas foi motivado primeiramente pela necessidade da economia matemática e em particular na procura de equiíbrio em trocas econômicas. Os primeiros trabalhos sobre isto, segundo Papageorgiou ([14]), são de K.Vind e Debreu-Schmeidler, a partir de então o assunto de multimedidas tem sido desenvolvido extensivamente,

Baseamo-nos nos trabalhos de Dimitrios A. Kandilakis ([9]), de C. Godet-Thobie, ([5] e [6]) e de R.Pallu de La Barriere ([12] e [13]).

O trabalho foi dividido em 4 capítulos. O capítulo 1 apresenta definições, notações e teoremas utilizados ao longo do texto. No capítulo 2 estudamos medida e multimedida. O capítulo 3 trata da extensão de uma multimedida e o capítulo 4, da integração com respeito a uma multimedida. 


\section{Capítulo 1}

\section{Preliminares}

O objetivo deste capítulo é apresentar definiçōes, notaçōes e teoremas utilizados neste trabalho. Enunciaremos apenas os principais resultados, uma vez que estamos considerando que o leitor tem algum conhecimento de Análise Funcional e de medidas escalares.

\subsection{Alguns resultados}

Vamos relembrar algumas propriedades dos espaços com os quais estaremos trabalhando.

Definição 1.1 Um espaço vetorial topológico é um espaço vetorial $X$ sobre um corpo IK com uma topologia em relação à qual

1. a aplicação de $X \times X \rightarrow X$ definida por $(x, y) \rightarrow x+y$ é contínua;

2. a aplicação de $\mathbb{I K} \times \mathrm{X} \rightarrow X$ definida por $(a, x) \rightarrow a . x$ é contínua.

Definição 1.2 Um espaço localmente convexo é um espaço vetorial topológico cuja topologia $\mathcal{T}$ é definida por uma família de seminormas $\mathcal{P}$ tal que $\underset{p \in \mathcal{P}}{\bigcap}\{x: p(x)=0\}=\{0\}$, na qual as sub-bases são os conjuntos $\{x \in X: p(x-y)<\varepsilon\}$, onde $p \in \mathcal{P}, y \in X$ e $\varepsilon>0$. Desta forma um subconjunto $\mathrm{U}$ de $\mathrm{X}$ é aberto se, e só se, para cada $w \in \mathrm{U}$, existem $p_{1}, \ldots p_{n} \in \mathcal{P}$ e $\varepsilon_{1}, \ldots, \Sigma_{n}$ tais que $\bigcap_{j=1}^{n}\left\{x \in \mathrm{X}: p_{j}(x-w)<\varepsilon_{j}\right\} \subset \mathrm{U}$.

Exemplo 1.3 Seja $X$ um espaço normado. Então $X$ é um espaço vetorial topológico e também um espaço localmente convexo. Se $X$ for completo diremos que $X$ é um espaço de Banach. Denotaremos por $X^{*}$ o espaço dos funcionais lineares contínuos sobre $X$. A inclusão canônica de $X$ em $X^{* *}$ será indicada por $J: X \longrightarrow X^{* *}$, onde $J(x)=\widehat{x}, \widehat{x}\left(x^{*}\right)=x^{*}(x)$, para todo $x^{*} \in X^{*}$. Usaremos também $\widehat{X}$ para $J(X)$. 
Exemplo 1.4 Seja $X$ um espaço normado. Para cada $x^{*} \in X^{*}$, definimos $p_{x^{*}}: X \rightarrow \mathbb{R}_{+}$por $p_{x^{*}}(x)=\left|x^{*}(x)\right|$, que é uma seminorma sobre $X$. A família $\mathcal{P}=\left\{p_{x^{*}}: x^{*} \in X^{*}\right\}$ torna $X$ um espaço localmente convexo. A topologia definida sobre $X$ por estas seminormas é chamada de topologia fraca, freqüentemente denotada por $\sigma\left(X, X^{*}\right)$ ou $\omega$. Denotaremos $(X, \omega)$ o espaço $X$ com a topologia $\omega$.

Exemplo 1.5 Seja $X$ um espaço normado. Para cada $x \in X$ definimos $p_{x}: X^{*} \rightarrow \mathbb{R}_{+}$por $p_{x}\left(x^{*}\right)=\left|x^{*}(x)\right|$, que é uma seminorma sobre $X^{*}$. A família $\mathcal{P}=\left\{p_{x}: x \in X\right\}$ torna $X^{*}$ um espaço localmente convexo. A topologia definida por estas seminormas é chamada de topologia fraca-estrela (ou fraca-*) sobre $X^{*}$, normalmente denotada por $\sigma\left(X^{*}, X\right)$ ou $\omega^{*}$. Denotaremos $\left(X^{*}, \omega^{*}\right)$ o espaço $X^{*}$ com a topologia $\omega^{*}$. Definimos $B_{X^{*}}=\left\{x^{*} \in X^{*}:\left\|x^{*}\right\|=1\right\}$.

Teorema 1.6 (Teorema de Hahn-Banach, forma geométrica ([3],p417)) Sejam $K_{1}$ e $K_{2}$ subconjuntos não vazios, convexos e disjuntos de um espaço localmente convexo real $X$. Se $K_{1}$ é compacto e $K_{2}$ é fechado, então existem constantes reais $c$ e $a$, e $x^{*} \in X^{*}$ tais que

$$
x^{*}(x)<c<a<x^{*}(y) \text {, para todo } x \in K_{1} \text { e } y \in K_{2} .
$$

Teorema 1.7 (Corolário do teorema de Hahn-Banach,forma analítica ([3],p65)) Se ja $X$ um espaço normado. Dado $x \in X$ não nulo existe $x^{*} \in X^{*}$ tal que $\left\|x^{*}\right\|=1$ e $x^{\star}(x)=\|x\|$.

Teorema 1.8 (Teorema de Krein-Smulian ([3],p429)) Sejam $X$ um espaço de Banach e $K$ um subconjunto convexo de $X^{*}$. O conjunto $K$ é fraco-* fechado se, e somente se, $K \cap r B_{X^{*}}$. é fraco-* fechado para todo $r \geq 0$.

Teorema 1.9 (Teorema de Eberlein-Smulian ([3],p431)) Seja A um subconjunto de um espaço de Banach $X$. As seguintes afirmações são equivalentes:

1. A é relativamente fraco sequencialmente compacto (ié, para toda seqüência $\left\{x_{n}\right\}$ em $A$, existe $\left\{x_{n_{k}}\right\} \omega$-convergente em $X$.)

2. $\bar{A}^{w}$ é fraco-compacto.

3. Todo subconjunto infinito de A tem um ponto limite fraco em X.

Definição 1.10 Sejam $X$ um espaço vetorial sobre $\mathbb{K}$ e $F \subset X$. Um subconjunto de $X$ não vazio $A$ é um subconjunto extremal de $F$ se, dados $x, y \in F \operatorname{com} t x+(1-t) y \in A$ para algum $t \in(0,1)$, então $x, y \in A$. Um subconjunto extremal de $F$ que só contenha um ponto é chamado de ponto extremal de $F$. Ao conjunto de pontos extremais de $F$ denotaremos ext $F$.

Exemplo 1.11 Os lados de um triângulo em $\mathbb{R}^{2}$ são subconjuntos extremais do mesmo, e seus vértices são pontos extremais dele. 
Teorema 1.12 (Teorema de Krein-Milman, ([3],p440)) Sejam $X$ um espaço localmente convexo e $K$ um subconjunto compacto de $X$. O conjunto dos pontos extremais de $K$ é não vazio e $K \subset \overline{c o}($ ext $K)$. Se $K$ for convexo, então $\overline{c o}($ ext $K)=K$.

Teorema 1.13 (Teorema de James ([8],p157)) Seja A um subconjunto limitado e fracofechado de um espaço de Banach real X. Se cada funcional contínuo em X assume seu supremo em A então A é fraco-compacto.

Teorema 1.14 (Teorema de Orlicz-Pettis ([4],p22)) Sejam $X$ espaço de Banach e $\left\{x_{n}\right\}$ seqüência em $X$. Se toda subsérie $\sum_{k} x_{n_{k}}$ for $\omega$-convergente, então toda subsérie $\sum_{k} x_{n_{k}}$ é convergente (em norma).

Teorema 1.15 (([10],p218)) Seja $\mathcal{F}$ uma família de funções de um conjunto $Z$ em um espaço topológico $X$. Para que $\mathcal{F}$ seja compacto relativo na topologia da convergência pontual de $X^{Z}$ é suficiente que:

1. $\mathcal{F}$ seja pontualmente fechada em $X^{Z}$,

2. para cada ponto $z$ de $Z$ o conjunto $\mathcal{F}[\{z\}]=\{f(z) / f \in \mathcal{F}\}$ tenha fecho compacto.

Se $X$ é um espaço de Hausdorff as condições acima também são necessárias.

\subsection{Funções convexas e funções semicontínuas infe- riormente}

Seja $X$ um espaço localmente convexo sobre $\mathbb{R}$.

Definição 1.16 A função $g: X \rightarrow \mathbb{R} \cup\{\infty\}$ é própria se existe $x \in X$ tal que $g(x)<+\infty$. Denotaremos por $g \not \equiv+\infty$.

Exemplo $1.17 f: \mathbb{R} \longrightarrow \mathbb{R}$ dada por $f(x)=\epsilon^{x}$.

Definição 1.18 Seja $X$ um espaço normado. Dada uma função própria $g: X \rightarrow \mathbb{R} \cup\{\infty\}$, chamamos de função conjugada de $g$ a função $g^{*}: X^{*} \longrightarrow \mathbb{R} \cup\{\infty\}$ dada por

$$
g^{*}\left(x^{*}\right)=\sup _{x \in X}\left\{x^{*}(x)-g(x)\right\}, x^{*} \in X^{*}
$$


Exemplo 1.19 Seja $f: \mathbb{R} \longrightarrow \mathbb{R}$ dada por $f(x)=e^{x}$. Por definição temos

$$
f^{*}\left(y^{*}\right)=\sup _{x \in \mathbb{R}}\left\{a x-e^{x}\right\} \text { onde } y^{*}(x)=a x \text { para algum } a \in \mathbb{R} \text {. }
$$

Analisemos o sinal de $a$. Se $a<0, a x-e^{x}$ pode ser arbitrariamente grande quando tomamos $x$ negativo, logo seu supremo é $+\infty$. Quando $a=0$, o supremo é trivialmente zero. Para $a>0$, um cálculo elementar pode ser usado para determinar o supremo, que resulta $a \cdot \log (a)-a$.

Portanto a função conjugada da função exponencial é:

$$
f^{*}\left(y^{*}\right)=\left\{\begin{array}{ll}
a \cdot \log (a)-a & \text { se } a>0 \\
0 & \text { se } a=0 \\
+\infty & \text { se } a<0
\end{array}, \text { onde } y^{*}(x)=a x .\right.
$$

Definição 1.20 A função $g: X \rightarrow \mathbb{R} \cup\{\infty\}$ é convexa se para $x, y \in X$ ocorrer que

$$
g(t x+(1-t) y) \leq t g(x)+(1-t) g(y) \text {, qualquer que seja } t \in(0,1) .
$$

Exemplo 1.21 $f: \mathbb{R} \longrightarrow \mathbb{R}$ dada por $f(x)=|x|$. conjuntos

Para uma função $g: X \rightarrow \mathbb{R} \cup\{\infty\}$ designaremos por epi $g$ e $[g \leq \lambda]$ os seguintes

$$
\begin{gathered}
\text { epi } g=\{(x, \lambda) \in X \times \mathbb{R} / \mathrm{g}(\mathrm{x}) \leq \lambda\} \\
{[g \leq \lambda]=\{x \in X / g(x) \leq \lambda\}}
\end{gathered}
$$

Proposição 1.22 (Propriedades de funções convexas,[2],p9) Consideramos que todas as funções abaixo estão definidas em $X$ a valores em $\mathbb{R} \cup\{\infty\}$.

1. Se $g$ é uma função convexa então epi $g$ é um conjunto convexo de $X \times \mathbb{R}$, e vale a recíproca.

2. Se $g$ é uma função convexa então para todo $\lambda$ em $\mathbb{R}$, o conjunto $[g \leq \lambda]$ é convexo, mas a recíproca é falsa.

3. Se $g_{1}$ e $g_{2}$ são convexas então $g_{1}+g_{2}$ é convexa.

4. Se $\left(g_{i}\right)_{i \in I}$ é uma família de funções convexas então as funções $f(x)=\sup _{i \in I} g_{i}(x)$ e $g(x)=$ $\sup _{h(x) \leq g_{i}(x)} h(x)$ são convexas.

Exemplo 1.23 Toda função $g^{*}$ é convexa.

Definição 1.24 Uma função $g: X \rightarrow \mathbb{R} \cup\{\infty\}$ é semicontínua inferiormente se para todo $\lambda \in \mathbb{R}$ o conjunto $[g \leq \lambda]=\{x \in X / g(x) \leq \lambda\}$ for fechado em $X$. 
Exemplo 1.25 $f: \mathbb{R} \longrightarrow \mathbb{R}$ dada por $f(x)=|x|, \mathbb{R}$ com a topologia usual.

Exemplo 1.26 Sejam $X$ espaço normado e $g: X \longrightarrow \mathbb{R}$ dada por $g(x)=\|x\|$. Então $g$ é uma função $\omega$-semicontínua inferiormente.

Definição 1.27 Uma função $f: X \rightarrow \mathbb{R} \cup\{\infty\}$ é indicadora de um conjunto $G \subset X$ quando

$$
f(x)=\left\{\begin{array}{l}
0, \text { se } x \in G \\
+\infty, \text { se } x \notin G .
\end{array}\right.
$$

Exemplo 1.28 Seja $K \subset X, K$ fechado. A função indicadora de $K$, denotada por $I_{K}$, é semicontínua inferiormente.

Proposição 1.29 (Propriedades de funções semicontínuas inferiormente,[2],p8) Con sideramos todas as funções abaixo definidas em $X$ a valores em $\mathbb{R} \cup\{\infty\}$

1. Se $g$ é semicontínua inferiormente então epi $g$ é fechado em $X \times \mathbb{R}$ e vale a recíproca.

2. A função $g$ é semicontínua inferiormente se, e somente se, para todo $x \in X \operatorname{com} g(x) \in \mathbb{R}$ e todo $\Xi>0$, existe uma vizinhança $V$ de $x$ tal que $g(y) \geq g(x)-\varepsilon$, para todo $y \epsilon$ $\mathrm{V}$, e para $x \in X$ com $g(x)=+\infty$, dado $M \in \mathbb{R}$, existe uma vizinhança $\mathrm{V}$ de $x$ tal que $g(y)>M$, para todo $y \in \mathrm{V}$.

3. Se $g$ é semicontínua inferiormente e $\left\{x_{\alpha}\right\}$ é um net convergente para $x$ então ocorre que $\lim \inf g\left(x_{\alpha}\right) \leq g(x)$

4. Se $g_{1}$ e $g_{2}$ são semicontínuas inferiormente então $g_{1}+g_{2}$ é semicontínua inferiormente.

5. Se $\left(g_{i}\right)_{i \in I}$ é uma família de funções semicontínuas inferiormente então as funções $f(x)=\sup _{i \in I} g_{i}(x)$ e $g(x)=\sup _{h(x) \leq g_{i}(x)} h(x)$ são semicontínuas inferiormente.

Observação 1.30 Sejam $X$ espaço normado e $g: X \rightarrow \mathbb{R} \cup\{\infty\}$ função própria. A aplicação $x^{*} \in X^{*} \mapsto x^{*}(x)-g(x) \in \mathbb{R}$ é $\|\cdot\|$-contínua, $\omega$-contínua e $\omega^{*}$-contínua para cada $x \in X$ com $g(x) \in \mathbb{R}$.

Proposição 1.31 Sejam $X$ espaço normado e $g: X \rightarrow \mathbb{R} \cup\{\infty\}$. Se $g \not \equiv+\infty$ então a função $g^{*}: X^{*} \longrightarrow \mathbb{R} \cup\{\infty\}$ dada por $g^{*}\left(x^{*}\right)=\sup _{x \in X}\left\{x^{*}(x)-g(x)\right\}$ é $\omega^{*}$-semicontínua inferiormente e convexa.

\section{prova:}

Resulta do exemplo 1.23, da proposiçào 1.29 e da observação 1.30 . 
Definição 1.32 Uma função $g: X \rightarrow \mathbb{R} \cup\{\infty\}$ é positivamente homogênea se ocorre $g(\lambda . x)=$ $\lambda g(x)$ para todo $\lambda>0$ e todo $x \in X$.

Proposição 1.33 Sejam $X$ espaço normado real e $g: X^{*} \longrightarrow \mathbb{R} \cup\{\infty\}$ convexa, própria, $\omega^{*}$ semicontínua inferiormente e positivamente homogênea. Então $g^{*}$ é indicadora de um conjunto $R \subset X^{* *}$ não vazio, convexo e $\omega^{*}$-fechado. Se $K \subset X$ for tal que $\widehat{K}=R \cap \widehat{X}, K$ é não vazio, convexo e $\omega$-fechado.

\section{prova:}

Sejam $x_{0}^{*} \in X^{*}$ e $\lambda_{0} \in \mathbb{R}$ com $g\left(x_{0}^{*}\right) \neq \infty$ e $g\left(x_{0}^{*}\right)>\lambda_{0}$. Consideremos $\left(X^{*}, \omega^{*}\right), \mathbb{R}$ com a topologia usual e $X^{*} \times \mathbb{R}$ com a topologia produto, que é localmente convexa.

Sendo $K_{1}=\left\{\left(x_{0}^{*}, \lambda_{0}\right)\right\}$ e $K_{2}=$ epi $g$, podemos aplicar o teorema 1.6 , pois ambos são convexos, não vazios, $K_{1}$ é compacto e $K_{2}$ é fechado em $X^{*} \times \mathbb{R}$.

Logo existem $f: X^{*} \times \mathbb{R} \rightarrow \mathbb{R}$ linear contínua e $\alpha \in \mathbb{R}$ com

$$
f\left(x_{0}^{*}, \lambda_{0}\right)<\alpha<f\left(x^{*}, \lambda\right)
$$

para qualquer $\left(x^{*}, \lambda\right) \in K_{2}$.

Como a aplicação $x^{*} \in X^{*} \mapsto f\left(x^{*}, 0\right) \in \mathbb{R}$ é uma forma linear $\omega^{*}$-contínua sobre $X^{*}$ então $f\left(x^{*}, 0\right)=x^{*}\left(x_{0}\right)$ para um certo $x_{0} \in X$.

Além disso, cada $\left(x^{*}, \lambda\right)$ pode ser escrito como $\left(x^{*}, \lambda\right)=\left(x^{*}, 0\right)+\lambda(0,1)$ e, colocando $f(0,1)=k$, temos $f\left(x^{*}, \lambda\right)=x^{*}\left(x_{0}\right)+\lambda k$, qualquer que seja $\left(x^{*}, \lambda\right) \in X^{*} \times \mathbb{R}$. y De (I) temos

$$
x_{0}^{*}\left(x_{0}\right)+k \lambda_{0}<\alpha \leq x^{*}\left(x_{0}\right)+k \lambda
$$

para todo $\left(x^{*}, \lambda\right) \in K_{2}$.

Como $\left(x_{0}^{*}, g\left(x_{0}^{*}\right)\right) \in K_{2}$, temos $x_{0}^{*}\left(x_{0}\right)+k \lambda_{0}<\alpha \leq x_{0}^{*}\left(x_{0}\right)+k g\left(x_{0}^{*}\right)$.

Assim, $k \cdot g\left(x_{0}^{*}\right)-k \cdot \lambda_{0}>0$. Mas $g\left(x_{0}^{*}\right)>\lambda_{0}$ e portanto $k>0$.

Pela definição $g^{*}\left(\frac{\widehat{-x_{0}}}{k}\right)=\sup _{x^{*} \in X^{*}}\left\{\frac{\widehat{-x_{0}}}{k}\left(x^{*}\right)-g\left(x^{*}\right)\right\}=\frac{1}{k} \sup _{x^{*} \in X^{*}}\left\{x^{*}\left(-x_{0}\right)-k g\left(x^{*}\right)\right\}$.

Usando que $x^{*}\left(-x_{0}\right)-k g\left(x^{*}\right)=-\left(x^{*}\left(x_{0}\right)+k g\left(x^{*}\right)\right) \stackrel{(\text { II })}{\leq}-\alpha$ pois $\left(x^{*}, g\left(x^{*}\right)\right) \in K_{2}$ para todo $x^{*} \in X^{*}$, temos $\sup _{x^{*} \in X^{*}}\left\{-x^{*}\left(x_{0}\right)-k g\left(x^{*}\right)\right\} \leq-\alpha$. Daí $g^{*}\left(\frac{-x_{0}}{k}\right) \leq \frac{-\alpha}{k}<\infty$, donde $\left.g^{*}\right|_{\widehat{X}} \not \equiv+\infty$.

Como $g$ é positivamente homogênea, vale que $g\left(x^{*}\right)=\lambda g\left(\lambda^{-1} \cdot x^{*}\right)$ para todo $\lambda>0$. Então

$$
\begin{aligned}
g^{*}\left(x^{* *}\right) & =\sup _{x^{*} \in X^{*}}\left\{x^{* *}\left(x^{*}\right)-g\left(x^{*}\right)\right\} \\
& =\lambda \cdot \sup _{x^{*} \in X^{* *}}\left\{\lambda^{-1} \cdot x^{* *}\left(x^{*}\right)-g\left(\lambda^{-1} \cdot x^{*}\right)\right\} \\
& =\lambda g^{*}\left(x^{* *}\right), \text { para todo } \lambda>0 .
\end{aligned}
$$


Logo $g^{*}$ só assume os valores 0 e $+\infty$, ou seja, $g^{*}$ é indicadora de um conjunto $R \subset X^{* *}$.

Podemos escrever $R=\left\{x^{* *} \in X^{* *} / g^{*}\left(x^{* *}\right) \leq 0\right\}$ e, sendo $g^{*}$ convexa e $\omega^{*}$-semicontínua inferiormente, $R$ é $\omega^{*}$-fechado e convexo, bem como não vazio pois mostramos acima que existe $\widehat{x_{0}} \in \widehat{X}$ tal que $g^{*}\left(\frac{\widehat{-x_{0}}}{k}\right) \in \mathbb{R}$ e então $g^{*}\left(\frac{\widehat{-x_{0}}}{k}\right)=0$.

Seja $K \subset X$ tal que $\widehat{K}=R \cap \hat{X}$, ou seja, $K=J^{-1}(R \cap \widehat{X})$. Então $K \neq \emptyset$ pois $\frac{-x_{0}}{k} \in K$.

Também $K$ é convexo por que $R$ e $\widehat{X}$ o são.

Ainda temos que $K$ é $\omega$-fechado pois se $\left\{y_{\alpha}\right\}$ em $K$ é $\omega$-convergente para $y_{0} \in X$, então $\left\{\widehat{y_{\alpha}}\right\}$ está em $R$ e é $\omega^{*}$-convergente para $\widehat{y_{0}}$. Como $R$ é $\omega^{*}$-fechado, $\widehat{y_{0}} \in R$ e portanto $y_{0} \in K$.

\section{3 Álgebras e $\sigma$-álgebras}

Seja $\Omega$ um conjunto.

Definição 1.34 Uma álgebra $\Sigma$ de subconjuntos de $\Omega$ é uma família de $\mathcal{P}(\Omega)$ tal que:

(i) $\emptyset \in \Sigma$;

(ii) $A \in \Sigma \Rightarrow .4^{c} \in \Sigma$;

(iii) dados quaisquer subconjuntos $A, B \in \Sigma$ ocorra $A \cup B \in \Sigma$.

Definição 1.35 Uma $\sigma$-álgebra $\Sigma$ de subconjuntos de $\Omega$ é uma família de $\mathcal{P}(\Omega)$ tal que:

(i) $\Sigma$ é uma álgebra;

(ii) para qualquer seqüência $\left\{A_{n}\right\} \in \Sigma$ de conjuntos, disjuntos dois a dois,y ocorrer que $\bigcup_{n \in \mathbb{N}} A_{n} \in \Sigma$.

Consideremos $\Sigma$ uma álgebra de subconjuntos de $\Omega, \Sigma_{1}$ a $\sigma$-álgebra gerada por $\Sigma$ e $\nu$ uma medida real nào-negativa $\sigma$-aditiva definida em $\Sigma_{1}$.

Dada d: $\Sigma_{1} \times \Sigma_{1} \longrightarrow \mathbb{R}_{+}$por $d(A, B)=\nu(A \triangle B)$, onde $A \triangle B=\left(A \cap B^{C}\right) \cup\left(B \cap A^{C}\right)$, temos que $d$ é uma pseudométrica ([11],p $73 \&$ \& 118).

Lema 1.36 Sejam $\left\{A_{n}\right\}$ e $\left\{F_{n}\right\}$ seqüências em $\mathcal{P}(\Omega)$. Então

(i) $\left(\bigcup_{n} A_{n}\right) \triangle\left(\bigcup_{n} F_{n}\right) \subset \bigcup_{n}\left(A_{n} \triangle F_{n}\right)$ e em particular $\left(A_{1} \cup A_{2}\right) \triangle\left(F_{1} \cup F_{2}\right) \subset\left(A_{1} \triangle F_{1}\right) \cup\left(A_{2} \triangle F_{2}\right)$;

(ii) $\left(A_{1} \cap A_{2}\right) \triangle\left(F_{1} \cap F_{2}\right) \subset\left(A_{1} \triangle F_{1}\right) \cup\left(A_{2} \triangle F_{2}\right)$.

prova: 
Por definiçào temos

$$
\left(\bigcup_{n} \cdot A_{n}\right) \triangle\left(\underset{k}{\cup} F_{k}\right)=\left(\left(\bigcup_{n} A_{n}\right) \cap\left(\underset{k}{\cup} F_{k}\right)^{c}\right) \cup\left(\left(\cup_{k} F_{k}\right) \cap\left(\bigcup_{n} A_{n}\right)^{c}\right)=\left(\left(\cup_{n} A_{n}\right) \cap\left(\underset{k}{\cap} F_{k}^{c}\right)\right) \cup\left(\left(\cup_{k} F_{k}\right) \cap\left(\cap_{n} A_{n}^{c}\right)\right) .
$$

Se $x \in\left(\underset{n}{\bigcup_{n}} A_{n}\right) \cap\left(\underset{k}{\cap} F_{k}^{c}\right)$ temos $x \in \underset{n}{\cup} A_{n}$ e $x \in \bigcap_{k} F_{k}^{c}$. Logo, existe um $n_{0} \in \mathbb{N}$ tal que $x \in A_{n_{0}}$ e $x \in F_{k}^{c}$ para todo $k \in \mathbb{N}$. Em particular $x \in A_{n_{0}} \cap F_{n_{0}}^{c}$ e portanto $x \in \cup_{n}\left(A_{n} \cap F_{n}^{c}\right)$.

Analogamente, se $x \in\left(\underset{k}{\cup} F_{k}\right) \cap\left(\cap_{n} A_{n}^{c}\right)$ temos $\left.x \in \underset{n}{\cup}\left(F_{n} \cap A_{n}^{c}\right)\right)$. Concluímos que se $x$ pertence a $\left(\bigcup_{n} A_{n}\right) \triangle\left(\cup_{k} F_{k}\right)$ então $x \in \bigcup_{n}^{\cup}\left(A_{n} \cap F_{n}^{c}\right)$ ou $x \in \cup_{n}\left(F_{n} \cap . A_{n}^{c}\right)$, ou seja, $x \in \cup_{n}\left(A_{n} \triangle F_{n}\right)$.

Para o item (ii) temos que

$$
\begin{aligned}
\left(A_{1} \cap A_{2}\right) \Delta\left(F_{1} \cap F_{2}\right)=\left[\left(A_{1} \cap A_{2}\right) \cap\left(F_{1} \cap F_{2}\right)^{c}\right] \cup\left[\left(F_{1} \cap F_{2}\right) \cap\left(A_{1} \cap A_{2}\right)^{c}\right]= \\
=\left[\left(A_{1} \cap A_{2}\right) \cap F_{1}^{c}\right] \cup\left[\left(A_{1} \cap A_{2}\right) \cap F_{2}^{c}\right] \cup\left[\left(F_{1} \cap F_{2}\right) \cap A_{1}^{c}\right] \cup\left[\left(F_{1} \cap F_{2}\right) \cap A_{2}^{c}\right]= \\
=\left[A_{2} \cap\left(A_{1} \cap F_{1}^{c}\right)\right] \cup\left[A_{1} \cap\left(A_{2} \cap F_{2}^{c}\right)\right] \cup\left[F_{1} \cap\left(F_{2} \cap A_{2}^{c}\right)\right] \cup\left[F_{2} \cap\left(F_{1} \cap A_{1}^{c}\right)\right] .
\end{aligned}
$$

Mas $A_{i} \cap F_{i}^{c} \subset A_{i} \triangle F_{i}, F_{i} \cap A_{i}^{c} \subset A_{i} \triangle F_{i}$ para $i=1,2$.

Logo $\left(A_{1} \cap A_{2}\right) \triangle\left(F_{1} \cap F_{2}\right) \subset\left(A_{1} \triangle F_{1}\right) \cup\left(A_{2} \triangle F_{2}\right)$.

Lema $1.37 \bar{\Sigma}^{d}$ é uma $\sigma$-álgebra de subconjuntos de $\Omega$.

prova:

Provaremos inicialmente que $\bar{\Sigma}^{d}$ é uma álgebra.

Dados $A, B \in \bar{\Sigma}^{d}$ queremos provar que $A \cup B \in \bar{\Sigma}^{d}$.

Para todo $\varepsilon>0$, existem $C^{\prime}, D \in \Sigma$ tais que $d\left(A, C^{\prime}\right)<\frac{\varepsilon}{2}$ e $d(B, D)<\frac{\varepsilon}{2}$.

Pelo lema $1.36(A \cup B) \triangle(C \cup D) \subset(A \triangle C) \cup(B \triangle D)$ e então temos $d(A \cup B, C \cup D)<\varepsilon$. Logo $A \cup B \in \bar{\Sigma}^{d}$.

Seja $\left\{A_{n}\right\}_{n \in \mathbb{N}}$ seqüência em $\bar{\Sigma}^{d}$.

Queremos mostrar que $B=\bigcup_{n \in \mathbb{N}} A_{n} \in \bar{\Sigma}^{d}$. Podemos admitir que $\left\{A_{n}\right\}$ é disjunta por que $\bar{\Sigma}^{d}$ é álgebra.

Para cada $k \in \mathbb{N}$, seja $B_{k}=A_{1} \cup A_{2} \cup \cdots \cup A_{k}$. Logo $B_{k} \in \bar{\Sigma}^{d}$.

Como $B_{k} \subset B=\bigcup_{n \in \mathbb{N}} A_{n}$ temos que $B \cap B_{k}{ }^{c}=\bigcup_{n=k+1}^{\infty} A_{n}$ e $B_{k} \cap B^{c}=\emptyset$. Então

$\mathrm{d}\left(B_{k}, B\right)=\nu\left(B \triangle B_{k}\right)=\nu\left(\bigcup_{n=k+1}^{\infty} A_{n}\right)=\sum_{n=k+1}^{\infty} \nu\left(A_{n}\right)$.

Sendo $\nu(B)<\infty$ temos $\sum_{n=k+1}^{\infty} \nu\left(A_{n}\right) \stackrel{k \longrightarrow \infty}{\longrightarrow} 0$ e voltando a (I) temos que $B \in \bar{\Sigma}^{d}$. 
Proposição $1.38 \Sigma$ é densa em $\Sigma_{1}$ com respeito a d.

prova:

Sabemos que $\bar{\Sigma}^{d} \subset \Sigma_{1}$. Mas $\Sigma \subset \bar{\Sigma}^{d}$ e $\bar{\Sigma}^{d}$ é $\sigma$-álgebra. Portanto $\bar{\Sigma}^{d} \supset \Sigma_{1}$. Logo $\bar{\Sigma}^{d}=\Sigma_{1}$ e $\Sigma$ é densa em $\Sigma_{1}$ com respeito a d. 


\section{Capítulo 2}

\section{Medida e multimedida}

Neste capítulo estaremos trabalhando com os conceitos de medidas e multimedidas vetoriais. Teremos um conjunto $\Omega$ fixado, um espaço de Banach real $X$ e usaremos álgebras ou $\sigma$-álgebras de $\mathcal{P}(\Omega)$.

\subsection{Medidas vetoriais e integração vetorial}

Seja $\Sigma$ uma álgebra de subconjuntos de $\Omega$.

Definição 2.1 1. Uma função $m: \Sigma \rightarrow X$ é medida vetorial se for finitamente aditiva, isto é, se dados $A$ e $B$ em $\Sigma$ disjuntos ocorrer que $m(A \cup B)=m(A)+m(B)$. Em particular $m(\emptyset)=0$. $f a(\Sigma, X)$.

2. O espaço de todas as medidas vetoriais de $\Sigma$ em $X$ será denotado por

Definição 2.2 1. Uma medida vetorial $m: \Sigma \rightarrow X$ é $\sigma$-aditiva se dada uma seqüência disjunta $\left\{A_{n}\right\}_{n \in \mathbb{N}}$ em $\Sigma$ com $\underset{n \in \mathbb{N}}{\cup} A_{n} \in \Sigma$ ocorrer $m\left(\bigcup_{n \in \mathbb{N}} A_{n}\right)=\sum_{n} m\left(A_{n}\right)$.

por $c a(\Sigma, X)$.

2. O espaço de todas as medidas vetoriais $\sigma$-aditivas de $\Sigma$ em $X$ será denotado

Definição 2.3 1. Seja $m: \Sigma \longrightarrow X$ medida vetorial. A variação de $m$ é a função não negativa $|m|: \Sigma \longrightarrow \mathbb{R} \cup\{\infty\}$ dada por $|m|(E)=\sup _{\pi} \sum_{A \in \pi}\|m(A)\|$ onde, para $E \in \Sigma$, o supremo é tomado sobre todas as partições finitas $\pi$ de $E$ tais que $\pi \subset \Sigma$.

2. Uma medida vetorial $m: \Sigma \longrightarrow X$ é de variação limitada se $|m|(\Omega)<\infty$.

3. O espaço de todas as medidas vetoriais de variação limitada de $\Sigma$ em $X$ será denotado por $b v(\Sigma, X)$. 
Observação 2.4 Seja $m$ medida vetorial.

monótona.

1.A função $|m|$ é medida (aditiva) de $\Sigma$ a valores em $[0,+\infty) \cup\{+\infty\}$ e é

2. Para todo $x^{*} \in X^{*}, x^{*}(m(\cdot))=x^{*} \circ m(\cdot)$ é medida (aditiva) a valores reais e é de variação limitada se $m$ o for. Sua variação $\left|x^{*} \circ m\right|(\cdot)$ é limitada por $\left\|x^{*}\right\||m|(\cdot)$.

Exemplo 2.5 Seja $T: L_{\infty}[0,1] \longrightarrow X$ um operador linear contínuo. Para cada conjunto $E$ contido em $[0,1]$ e Lebesgue-mensurável, definamos $m(E)=T\left(\chi_{E}\right)$, onde $\chi_{E}$ é a função característica de $E$. Pela linearidade de $T, m$ é uma medida vetorial finitamente aditiva mas não é $\sigma$-aditiva em geral, mesmo no caso de $X=\mathbb{R}$.

Exemplo 2.6 Seja $T: L_{1}[0,1] \longrightarrow X$ um operador linear contínuo. Novamente definimos $m(E)=T\left(\chi_{E}\right)$, onde $\chi_{E}$ é a função característica de $E$ para cada conjunto $E$ contido em $[0,1]$ e Lebesgue-mensurável. Então $m$ é evidentemente finitamente aditiva. Além disso, para cada $E$, temos $\|m(E)\| \leq \lambda(E)\|T\|$, onde $\lambda$ é a medida de Lebesgue do $[0,1]$. Como $m$ é dominada por $\lambda$ e é finitamente aditiva, $m$ é $\sigma$-aditiva.

Exemplo 2.7 Seja $m \in b v(\Sigma, X)$. Se $f$ é uma função simples mensurável sobre $\Omega$, digamos $f=\sum_{i=1}^{n} \alpha_{i} \chi_{E_{i}}$, onde $\alpha_{i}$ são escalares não nulos e $E_{i}$ são elementos de $\Sigma$ disjuntos dois a dois, $1 \leq i \leq n$, definimos $T_{m}(f)=\sum_{i=1}^{n} \alpha_{i} m\left(E_{i}\right)$. Esta função é uma aplicação linear do espaço das funções simples mensuráveis em $X$. Além disso, se $f$ é como acima e $\beta=\sup \{|f(z)|: z \in \Omega\}$, então temos que

$$
\begin{aligned}
\left\|T_{m}(f)\right\| & =\left\|\sum_{i=1}^{n} \alpha_{i} m\left(E_{i}\right)\right\| \\
& =\beta\left\|\sum_{i=1}^{n}\left(\frac{\alpha_{i}}{\beta}\right) m\left(E_{i}\right)\right\| \\
& \leq \beta \sum_{i=1}^{n}\left\|\frac{\alpha_{i}}{\beta} m\left(E_{i}\right)\right\| \\
& \leq \beta \sup _{E \in \Pi}\|m(E)\| \\
& =\beta|m|(\Omega) .
\end{aligned}
$$

Como $m \in b v(\Sigma, X)$, temos que $T_{m}$ é uma aplicação linear contínua com $\left\|T_{m}\right\| \leq|m|(\Omega)$, se no conjuntos das funçōes simples mensuráveis usamos a norma $\|f\|_{\infty}=\sup _{z \in \Omega}|f(z)|$.

Seja $f$ uma função que é limite uniforme de funções simples mensuráveis, digamos $\left\{s_{k}\right\}$. Usando a norma do supremo temos $\left\|T_{m}\left(s_{k}-s_{j}\right)\right\| \leq\left\|s_{k}-s_{j}\right\|_{\infty}|m|(\Omega)$.

Portanto a seqüência $\left\{T_{m}\left(s_{k}\right)\right\}$ é de Cauchy, logo convergente para algum $x_{0} \in X$. Defini$\operatorname{mos} T_{m}(f)=x_{0}$. Note-se que $x_{0}$ nào depende da particular seqüência $\left\{s_{k}\right\}$ escolhida e ainda temos $\left\|T_{m}\right\| \leq\|f\|_{\infty}|m|(\Omega)$ 
Assim $T_{m}$ admite uma única extensào linear contínua para o conjunto das funções que são limite uniforme de funçōes simples mensuráveis, que continuaremos chamando de $T_{m}$.

Denotaremos por $B(\Sigma)$ o conjunto das funções $f: \Omega \longrightarrow \mathbb{R}$ limitadas e mensuráveis. Observemos que $B(\Sigma)$ é um subconjunto do conjunto de funções que são limite uniforme de funções simples. Assim podemos definir $T_{m}$ para as funções de $B(\Sigma)$.

Definição 2.8 Sejam $f \in B(\Sigma)$ e $m \in b v(\Sigma, X)$. Definimos a integral de Bartle de $f$ em relação a $m$ em $\Omega$ por $\int_{\Omega} f d m=T_{m}(f)$, onde $T_{m}: B(\Sigma) \longrightarrow X$ é como no exemplo 2.7. Também definimos, para $A \in \Sigma, \int_{A} f d m=\int_{\Omega} f \chi_{A} d m=T_{m}\left(f \chi_{A}\right)$.

Proposição 2.9 Sejam $f, g \in B(\Sigma)$ e $m, n \in b v(\Sigma, X)$ e $A \in \Sigma$. Valem:

1. $\int_{A}(f+g) d m=\int_{A} f d m+\int_{A} g d m$.

2. $\int_{A} f d(m+n)=\int_{A} f d m+\int_{A} f d n$.

3. $\left\|\int_{A} f d m\right\| \leq\|f\|_{\infty}|m|(A)$.

4. Se $\left\{m_{\alpha}\right\}$ é net em $b v(\Sigma, X)$ com $\left|m_{\alpha}\right|(\Omega) \leq M \in \mathbb{R}$ para todo $\alpha, m_{0} \in b v(\Sigma, X)$, e $m_{\alpha}(B) \longrightarrow m_{0}(B)$ para todo $B \in \Sigma, B \subset A$, então $\int_{A} f d m_{\alpha} \longrightarrow \int_{A} f d m_{0}$.

prova:

(1) Temos $\int_{A}(f+g) d m=\int_{\Omega}(f+g) \chi_{A} d m=T_{m}\left((f+g) \chi_{A}\right)$ por definição. Sabemos que $T_{m}$ é aplicação linear. Logo $T_{m}\left((f+g) \chi_{A}\right)=T_{m}\left(f \chi_{A}+g \chi_{A}\right)=T_{m}\left(f \chi_{A}\right)+T_{m}\left(g \backslash \backslash_{A}\right)=$ $\int_{A} f d m+\int_{A} g d m$.

(2) Temos $m+n \in b v(\Sigma, X)$. Suponhamos que $f$ seja simples, $f=\sum_{i=1}^{n} \alpha_{i} \chi_{E_{i}}$, onde $\alpha_{i}$ são escalares não nulos e $E_{i} \in \Sigma$, dois a dois disjuntos, $1 \leq i \leq n$. Então

$$
\begin{array}{rlrl}
\int_{A} f d(m+n) & =\int_{\Omega} f \chi_{A} d(m+n) & & \sum_{i=1}^{n} \alpha_{i}(m+n)\left(E_{i} \cap A\right) \\
& =\sum_{i=1}^{n} \alpha_{i}\left(m\left(E_{i} \cap A\right)+n\left(E_{i} \cap A\right)\right) & =\sum_{i=1}^{n}\left[\alpha_{i} m\left(E_{i} \cap A\right)+\alpha_{i} n\left(E_{i} \cap A\right)\right] \\
& =\sum_{i=1}^{n} \alpha_{i} m\left(E_{i} \cap A\right)+\sum_{i=1}^{n} \alpha_{i} n\left(E_{i} \cap A\right) & =\int_{A} f d m+\int_{A} f d n .
\end{array}
$$

Se $f$ não for simples, como $f \in B(\Sigma)$, existe uma seqüência de funções simples mensuráveis $\left\{s_{k}\right\}$ tal que $s_{k} \stackrel{u}{\longrightarrow} f$.

Entào $T_{m}\left(s_{k}\right) \longrightarrow T_{m}(f), T_{n}\left(s_{k}\right) \longrightarrow T_{n}(f), T_{m+n}\left(s_{k}\right) \longrightarrow T_{m+n}(f)$ e como $T_{m+n}\left(s_{k}\right)=$ $T_{m}\left(s_{k}\right)+T_{n}\left(s_{k}\right)$, para todo $k \in \mathbb{N}$, temos $T_{m+n}(f)=T_{m}(f)+T_{n}(f)$. 
(3) Se $f$ for simples. digamos $f=\sum_{i=1}^{n} \alpha_{i} \chi_{E_{i}}$, onde $\alpha_{i}$ são escalares não nulos e $E_{i} \in \Sigma$, dois a dois disjuntos, $1 \leq i \leq n$, temos

$$
\left\|\int_{A} f d m\right\|=\left\|\sum_{i=1}^{n} \alpha_{i} m\left(E_{i} \cap A\right)\right\| \leq\|f\|_{\infty} \sum_{i=1}^{n}\left\|m\left(E_{i} \cap A\right)\right\| \leq\|f\|_{\infty}|m|(A) .
$$

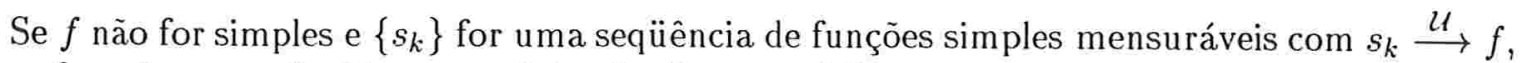
temos $\int_{A} s_{k} d m \longrightarrow \int_{A} f d m$ e também $\left\|s_{k}\right\|_{\infty} \longrightarrow\|f\|_{\infty}$. Pela parte anterior, segue-se que $\left\|\int_{A} f d m\right\| \leq\|f\|_{\infty}|m|(A)$.

(4) Se $f$ for simples. dada por $f=\sum_{i=1}^{n} \alpha_{i} \chi_{E_{i}}$, onde $\alpha_{i}$ são escalare não nulos e $E_{i} \in \Sigma$, dois a dois disjuntos, $1 \leq i \leq n$, então $\left\|\int_{A} f d m_{\alpha}-\int_{A} f d m_{0}\right\| \leq \sum_{i=1}^{n}\left|\alpha_{i}\right|\left\|m_{\alpha}\left(E_{i} \cap A\right)-m_{0}\left(E_{i} \cap A\right)\right\| \longrightarrow 0$.

Se $f$ não for simples, dado $\varepsilon>0$, existe $s$ simples mensurável com $\|f-s\|_{\infty}<\varepsilon$. Com o mesmo $\varepsilon>0$, existe $\alpha_{0}$ tal que $\alpha>\alpha_{0}$ implica em $\left\|\int_{A} s d m_{\alpha}-\int_{A} s d m_{0}\right\|<\varepsilon$.

Então, se $\alpha>\alpha_{0}$,

$$
\begin{aligned}
\left\|\int_{A} f d m_{\alpha}-\int_{A} f d m_{0}\right\| & \leq\|f-s\|_{\infty}\left[\left|m_{\alpha}\right|(A)+\left|m_{0}\right|(A)\right]+\left\|\int_{A} s d m_{\alpha}-\int_{A} s d m_{0}\right\| \\
& <\varepsilon\left[M+\left|m_{0}\right|(A)+1\right] .
\end{aligned}
$$

$\operatorname{Logo} \int_{A} f d m_{\alpha} \longrightarrow \int_{A} f d m_{0}$.

Proposição 2.10 Para $f \in B(\Sigma)$ e $m \in b v(\Sigma, X)$, a função $n: \Sigma \longrightarrow X$, dada por $n(A)=\int_{A} f d m, A \in \Sigma$, é uma medida vetorial de variação limitada com $|n|(\cdot) \leq\|f\|_{\infty}|m|(\cdot)$ e ainda para todo $x^{*} \in X^{*}$ vale $x^{*}(n(A))=\int_{A} f d x^{*} \circ m$.

\section{prova:}

Sejam $f \in B(\Sigma)$ e $m \in b v(\Sigma, X)$. Temos $n(\emptyset)=\int_{\emptyset} f d m=0$.

Dados $A, B \in \Sigma$ disjuntos, então

$$
\begin{aligned}
& n(A \cup B)=\quad \int_{A \cup B} f d m=\int_{\Omega} f \cdot \chi_{A \cup B} d m \\
& =\int_{\Omega} f \chi_{A} d m+\int_{\Omega} f \chi_{B} d m=\int_{A} f d m+\int_{B} f d m \\
& =n(A)+n(B) \text {. }
\end{aligned}
$$

Logo $n$ é aditiva.

Pela proposição $2.9(3),\|n(E)\|=\left\|\int_{E} f d m\right\| \leq\|f\|_{\infty}|m|(E)$, para todo $E \in \Sigma$.

Fixemos $A \in \Sigma$.

Sabemos que $f \in B(\Sigma)$. Então existe um $M \in \mathbb{R}$ tal que $\|f\|_{\infty}<M$ e como $m \in b v(\Sigma, X)$ temos que $|m|(A) \leq|m|(\Omega)<\infty$.

Por outro lado $|n|(A)$ satisfaz 


$$
\begin{aligned}
|n|(A) & =\sup _{\pi} \sum_{E \in \pi}\|n(E)\| \\
& \leq \sup _{\pi} \sum_{E \in \pi}\|f\|_{\infty}|m|(E) \\
& \leq\|f\|_{\infty} \sup _{\pi} \sum_{E \in \pi}|m|(E) \\
& \leq\|f\|_{\infty}|m|(A) \\
& \leq M|m|(\Omega)<\infty .
\end{aligned}
$$

Sejam $x^{*} \in X^{*}$ e $f \in B(\Sigma)$.

Se $f$ for simples, dada por $f=\sum_{i=1}^{n} \alpha_{i} \chi_{E_{i}}$, onde $\alpha_{i}$ são escalares não nulos e $E_{i} \in \Sigma$,dois a dois disjuntos, $1 \leq i \leq n$, temos que

$$
x^{*}\left(\int_{A} f d m\right)=x^{*}\left(\sum_{i=1}^{n} \alpha_{1} m\left(E_{i} \cap A\right)\right)=\sum_{i=1}^{n} \alpha_{i} x^{*}\left(m\left(E_{i} \cap A\right)\right)=\int_{A} f d x^{*} \circ m .
$$

Se $f$ não for simples, como $f \in B(\Sigma)$, existe seqüência de funções simples mensuráveis tal que $s_{k} \stackrel{\mathcal{u}}{\longrightarrow} f$.

Para cada $s_{k}$ vale $x^{*}\left(\int_{A} s_{k} d m\right)=\int_{A} s_{k} d x^{*} \circ m$, e $\int_{A} s_{k} d x^{*} \circ m \longrightarrow \int_{A} f d x^{*} \circ m$ e ainda $x^{*}\left(\int_{A} s_{k} d m\right) \longrightarrow x^{*}\left(\int_{A} f d m\right)$.

Obtemos que $x^{*}\left(\int_{A} f d m\right)=\int_{A} f d x^{*} \circ m$, ou seja, $x^{*}(n(A))=\int_{A} f d x^{*} \circ m$.

\subsection{Multimedidas} juntos

Seja $\Sigma$ uma álgebra de subconjuntos de $\Omega$, consideraremos em $\mathcal{P}(X)$ os seguintes subcon-

$$
\begin{aligned}
P_{0}(X) & =\{A \subset X / A \neq \emptyset\} \\
P_{f c}(X) & =\{A \subset X / A \neq \emptyset, \text { fechado e convexo }\}, e \\
P_{l f c}(X) & =\{A \subset X / A \neq \emptyset, \text { limitado, fechado e convexo }\}, e \\
P_{w k c}(X) & =\{A \subset X / A \neq \emptyset, \text { fracamente compacto e convexo }\}
\end{aligned}
$$

Definição 2.11 1.Dizemos que uma multifunção $M: \Sigma \longrightarrow P_{0}(X)$ é uma multimedida se $M(\emptyset)=\{0\}$ e se for finitamente aditiva, isto é, se dados $A$ e $B$ em $\Sigma$ disjuntos ocorrer que $M(A \cup B)=M(A)+M(B)$.

2.Uma multimedida $M$ é $\sigma$-aditiva ou multimedida forte se dada uma seqüência disjunta $\left\{A_{n}\right\}_{n \in \mathbb{N}}$ em $\Sigma$, com $\underset{n \in \mathbb{N}}{\cup} A_{n} \in \Sigma$, ocorrer que se para todo seqüência $\left\{x_{n}\right\}$ com 
$x_{n} \in M\left(A_{n}\right), n \in \mathbb{N}$, a série $\sum_{n \in \mathbb{N}} x_{n}$ converge e $M\left(\cup_{n \in \mathbb{N}} A_{n}\right)=\sum_{n \in \mathbb{N}} M\left(A_{n}\right)$, onde $\sum_{n \in \mathbb{N}} M\left(A_{n}\right)=$ $\left\{x / x=\sum_{n \in \mathbb{N}} x_{n}, x_{n} \in M\left(A_{n}\right)\right\}$.

3.Uma multimedida $M$ é fortemente aditiva se para cada seqüência disjunta $\left\{A_{n}\right\}_{n \in \mathbb{N}}$ em $\Sigma$ e cada seqüência $\left\{x_{n}\right\}$ com $x_{n} \in M\left(A_{n}\right), n \in \mathbb{N}$, a série $\sum_{n \in \mathbb{N}} x_{n}$ converge.

4.Uma multifunção $M: \Sigma \longrightarrow P_{0}(X)$ é uma multimedida fraca se para cada $x^{*} \in X^{*}$, a função $\sigma\left(x^{*}, M(\cdot)\right): \Sigma \longrightarrow \mathbb{R} \cup\{\infty\}$ dada por $\sigma\left(x^{*}, M(A)\right)=\sup _{x \in M(A)} x^{*}(x)$ for uma medida $\sigma$-aditiva com sinal.

5. Em todos os casos anteriores definimos $M(\Sigma)=\cup_{A \in \Sigma} M(A)$.

Observação 2.12 As séries do tipo $\sum_{n \in \mathbb{N}} x_{n}, x_{n} \in M\left(A_{n}\right)$, onde $\left\{A_{n}\right\}_{n \in \mathbb{N}}$ é uma seqüência disjunta em $\Sigma$ e $M$ é multimedida $\sigma$-aditiva ou fortemente aditiva são incondicionalmente convergentes em $X$, pois a convergência independe da ordem $\operatorname{dos} A_{n^{\prime} s}$.

Exemplo 2.13 (Multimedida forte) $\operatorname{Sejam~}(\Omega, \Sigma, \lambda)$ um espaço mensurável com $\lambda$ medida positiva, $X$ um espaço de Banach e $Q$ um subconjunto limitado de $X$. Se $\Xi$ é uma família de medidas vetoriais de $\Sigma$ em $X$ satisfazendo que para cada $m \in \Xi$, para cada $A \in \Sigma$ ocorre que $m(A) \in \lambda(A) . Q$. Definimos $M$ por $M(A)=\left\{\sum_{i \leq n} m_{i}\left(A_{i}\right), m_{i} \in \Xi,\left\{A_{i}\right\}_{i \leq n}\right.$ partição finita de $\left.A\right\}$. Então $M$ é uma multimedida forte.

Exemplo 2.14 (Multimedida fraca que não é forte) Sejam $X=\mathbb{R}^{2}$ e $\mathcal{C}$ o gráfico de $y=(x-1)^{2}$. Tomemos $\Omega=\mathbb{N}$ e $\Sigma=\mathcal{P}(\mathbb{N})$. Seja a multifunção $M: \Sigma \longrightarrow P_{0}(X)$ definida por

$$
M(A)=\left\{\begin{array}{rr}
\sum_{n \in A} \Delta(n) & , \text { se } A \text { finito } \\
\mathbb{R}^{2} & \text {, se } A \text { infinito }
\end{array}\right.
$$

Onde $\Delta(n)$ é dada por:

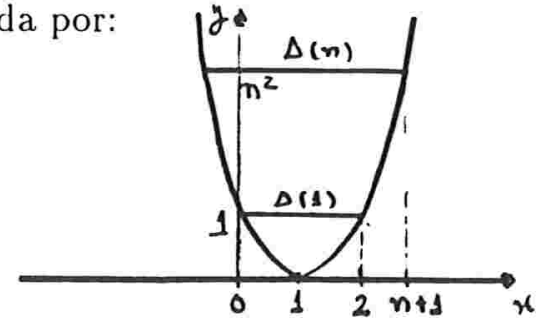

Para cada $z \in \mathbb{R}^{2}$ temos $\lim _{n \rightarrow \pm \infty} \sup _{z \in \Delta(n)} x^{*}(z)=+\infty$. Então, para cada cada $z \in \mathbb{R}^{2}$, para cada $A=\bigcup_{n} A_{n}$ (disjuntos), $\sigma(z, M(A))=\sum_{n \in \mathbb{N}} \sigma\left(z, M\left(A_{n}\right)\right)$. Mas. para cada $x_{n} \in M\left(A_{n}\right), x_{n}$ não é somável.

Proposição 2.15 1.Se $M: \Sigma \longrightarrow P_{0}(X)$ é uma multimedida forte então $M$ é uma multimedida fraca. 
2. Se $M: \Sigma \longrightarrow P_{f c}(X)$ é uma multifunção com $\sigma\left(x^{*}, M(\cdot)\right)$ medida aditiva para todo $x^{*} \in X^{*}$ entào $M(\emptyset)=\{0\}$ e se $A, B \in \Sigma$ são disjuntos, $\overline{M(A)+M(B)}=M(A \cup B)$. (aditiva) para todo $x^{*} \in X^{*}$.

3. Se $M: \Sigma \longrightarrow P_{0}(X)$ é uma multimedida então $\sigma\left(x^{*}, M(\cdot)\right)$ é medida

prova:

(1)Seja $x^{*} \in X^{*}$ fixado e consideremos a função $\mu: \Sigma \rightarrow \overline{\mathbb{R}}$ dada por

$$
\mu(A)=\sigma\left(x^{*}, M(A)\right)=\sup _{x \in M(A)} x^{*}(x) .
$$

Temos:

(i) $\mu$ só assume valores em $\mathbb{R} \cup\{\infty\}$.

Como nenhum $M(A)$ é vazio, teremos sempre $\sup _{x \in M(A)} x^{*}(x)>-\infty$.

(ii) $\mu(\emptyset)=0$.

De fato, $\mu(\emptyset)=\sigma\left(x^{*}, M(\emptyset)\right)=\sup _{x \in\{0\}} x^{*}(x)=0$

(iii) $\mu\left(\cup_{n \in \mathbb{N}} A_{n}\right)=\sum_{n \in \mathbb{N}} \mu\left(A_{n}\right)$, para toda seqüência disjunta $\left\{A_{n}\right\}_{n \in \mathbb{N}}$ em $\Sigma$ com $\underset{n \in \mathbb{N}}{\cup} A_{n} \in \Sigma$.

Seja uma seqüência disjunta $\left\{A_{n}\right\}_{n \in \mathbb{N}}$ em $\Sigma$ com $\bigcup_{n \in \mathbb{N}} A_{n} \in \Sigma$.

Como $\mu(A)=\sigma\left(x^{*}, M(A)\right)=\sup _{x \in M(A)} x^{*}(x)$, dado $\varepsilon>0$ existem $\overline{x_{n}} \in M\left(A_{n}\right)$ tais que $x^{*}\left(\overline{x_{n}}\right)>\mu\left(A_{n}\right)-\frac{\varepsilon}{2^{n}}$, ou seja, $\mu\left(A_{n}\right)<x^{*}\left(\overline{x_{n}}\right)+\frac{\varepsilon}{2^{n}}, n \in \mathbb{N}$.

$\operatorname{Assim}, \sum_{n=1}^{k} \mu\left(A_{n}\right) \leq \sum_{n=1}^{k} x^{*}\left(\overline{x_{n}}\right)+\varepsilon\left(1-\frac{1}{2^{k}}\right)$.

Tomando o limite superior temos $\limsup \sum_{n=1}^{k} \mu\left(A_{n}\right) \leq \limsup \sum_{n=1}^{k} x^{*}\left(\overline{x_{n}}\right)+\lim \sup \varepsilon\left(1-\frac{1}{2^{k}}\right)$.

Como $M$ é multimedida forte a série $\sum_{n \in \mathbb{N}} \overline{x_{n}}$ converge, e então $\sum_{n \in \mathbb{N}} x^{*}\left(\overline{x_{n}}\right)$ converge. Portanto $\lim \sup \sum_{n=1}^{k} x^{*}\left(\overline{x_{n}}\right)=\sum_{n \in \mathbb{N}} x^{*}\left(\overline{x_{n}}\right)$.

Com isto temos $\limsup \sum_{n=1}^{k} \mu\left(A_{n}\right) \leq \sum_{n \in \mathbb{N}} x^{*}\left(\overline{x_{n}}\right)+\varepsilon=x^{*}\left(\sum_{n \in \mathbb{N}} \overline{x_{n}}\right)+\varepsilon$, de onde segue que $\limsup \sum_{n=1}^{k} \mu\left(A_{n}\right) \leq \mu\left(\bigcup_{n \in \mathbb{N}} A_{n}\right)+\varepsilon$, pois $\mu\left(\bigcup_{n \in \mathbb{N}} A_{n}\right)=\sup _{x_{n} \in M\left(A_{n}\right)} x^{*}\left(\sum_{n \in \mathbb{N}} x_{n}\right)$.

Como $\varepsilon$ é arbitrário.

$$
\limsup \sum_{n=1}^{k} \mu\left(A_{n}\right) \leq \mu\left(\cup_{n \in \mathbb{N}} A_{n}\right) .
$$


Por outro lado, para quaisquer $x_{n} \in M\left(A_{n}\right)$ e para cada $k \in \mathbb{N}$ temos que

$$
\sum_{n=1}^{k} x^{*}\left(x_{n}\right) \leq \sum_{n=1}^{k} \sup _{x_{n} \in M\left(A_{n}\right)} x^{*}\left(x_{n}\right)=\sum_{n=1}^{k} \mu\left(A_{n}\right) .
$$

$\operatorname{Assim} \liminf _{k} \sum_{n=1}^{k} x^{*}\left(x_{n}\right) \leq \liminf _{k} \sum_{n=1}^{k} \mu\left(A_{n}\right)$.

Mas $\liminf _{k} \sum_{n=1}^{k} x^{*}\left(x_{n}\right)=\sum_{n \in \mathbb{N}} x^{*}\left(x_{n}\right)=x^{*}\left(\sum_{n} x_{n}\right)$, como antes, por $M$ ser multimedida forte. Logo,

$$
\mu\left(\cup_{n} A_{n}\right)=\sup _{x_{n} \in M\left(A_{n}\right)} x^{*}\left(\sum_{n=1}^{k} x_{n}\right) \leq \liminf \sum_{n=1}^{k} \mu\left(A_{n}\right)
$$

De (I) e (II) temos $\mu\left(\cup_{n \in \mathbb{N}} A_{n}\right)=\lim _{k \rightarrow \infty} \sum_{n=1}^{k} \mu\left(A_{n}\right)$, ou seja, $\mu\left(\cup_{n \in \mathbb{N}} A_{n}\right)=\sum_{n \in \mathbb{N}} \mu\left(A_{n}\right)$.

Logo $\mu$ é uma medida $\sigma$-aditiva com sinal e portanto $M$ é uma multimedida fraca.

(2) Se $x \in M(\emptyset)$ e $x \neq 0$, existe $x_{0}^{*} \in X^{*} \operatorname{com} x_{0}^{*}(x)=\|x\|>0 . \quad \operatorname{Logo} \sigma\left(x_{0}^{*}, M(\emptyset)\right)>0 \mathrm{e}$ $\sigma\left(x_{\mathbf{0}}^{*}, M(\cdot)\right)$ não seria medida aditiva.

Sejam $A, B \in \Sigma$ disjuntos. Sabemos que $M(A \cup B)$ é convexo e fechado. Para $x=x_{A}+x_{B} \in M(A)+M(B)$, se $x \notin M(A \cup B)$, pelo teorema 1.6 existem $c, a \in \mathbb{R}$
e $x_{0}^{*} \in X^{*}$, com

$x_{0}^{*}(x)=x_{0}^{*}\left(x_{A}\right)+x_{0}^{*}\left(x_{B}\right)>c>a>x_{0}^{*}(z)$, para todo $z \in M(A \cup B)$.

Portanto,

$$
\sigma\left(x_{0}^{*}, M(A)\right)+\sigma\left(x_{0}^{*}, M(B)\right) \geq x_{0}^{*}(x)>c>a \geq \sigma\left(x_{0}^{*}, M(A \cup B)\right),
$$

e $\sigma\left(x_{0}^{*}, M(\cdot)\right)$ não seria aditiva. Logo,

$$
M(A)+M(B) \subset M(A \cup B) .
$$
com

Por outro lado, se existir $y \in M(A \cup B)$ com $y \notin \overline{M(A)+M(B)}$, de novo existirá $y_{0}^{*} \in X^{*}$

$y_{0}^{*}(y)>c>a>y_{0}^{*}(w)$, para todo $w \in \overline{M(A)+M(B)}$ e daí

$$
\sigma\left(y_{0}^{*}, M(A \cup B)\right) \geq y_{0}^{*}(y)>c>a \geq \sigma\left(y_{0}^{*}, M(A)\right)+\sigma\left(y_{0}^{*}, M(B)\right),
$$

contrariando também a aditividade de $\sigma\left(y_{0}^{*}, M(\cdot)\right)$.

Logo, 


$$
M(A \cup B) \subset \overline{M(A)+M(B)} .
$$

De (III) e (IV) segue que $M(A \cup B)=\overline{M(A)+M(B)}$.

(3) Como $M(\emptyset)=\{0\}, \sigma\left(x^{*}, M(\emptyset)\right)=0$, para tódo $x^{*} \in X^{*}$.

Dados $A, B \in \Sigma$ disjuntos, temos que $M(A \cup B)=M(A)+M(B)$.

Logo, para todo $x^{*} \in X^{*}$,

$$
\begin{aligned}
\sigma\left(x^{*}, M(A \cup B)\right)= & \sup _{x \in M(A \cup B)} x^{*}(x) \\
= & \sup ^{x=x_{A}+x_{B}} x^{*}\left(x_{A}+x_{B}\right) \\
& =\sup _{A} \in M(A), x_{B} \in M(B) \\
& x^{*}\left(x_{A}\right)+\sup _{x_{b} \in M(B)} x^{*}\left(x_{B}\right) \\
= & \sigma\left(x^{*}, M(A)\right)+\sigma\left(x^{*}, M(B)\right) .
\end{aligned}
$$

Lema 2.16 Seja $M: \Sigma \longrightarrow P_{0}(X)$ uma multimedida. $M$ é fortemente aditiva se e somente se para toda seqüência disjunta $\left\{A_{n}\right\}_{n \in \mathbb{N}}$ em $\Sigma$ e quaisquer que sejam $x_{n} \in M\left(A_{n}\right), n \in \mathbb{N}$ temos que $\lim _{n \rightarrow \infty}\left\|x_{n}\right\|=0$.

\section{prova:}

Se $M$ é fortemente aditiva, dados $\left\{A_{n}\right\}$ e $\left\{x_{n}\right\}$ como no enunciado, a série $\sum_{n \in \mathbb{N}} x_{n}$ converge e portanto $\lim _{n \longrightarrow \infty}\left\|x_{n}\right\|=0$.

Suponhamos que $M$ nào seja fortemente aditiva. Entào existem $\left\{A_{j}\right\}_{j \in \mathbb{N}}$ em $\Sigma$ e $x_{j}$ em $M\left(A_{j}\right)$ tais que a série $\sum_{j \in \mathbb{N}} x_{j}$ é divergente.

Logo $\left\{s_{k}\right\}=\left\{\sum_{j=1}^{k} x_{j}\right\}$ não é de Cauchy, ou seja, existe $\varepsilon_{0}>0$ tal que para todo $n_{0}$ existem $m, n>n_{0}$ com $\left\|s_{m}-s_{n}\right\|>\sum_{0}$.

Sem perda de generalidade podemos supor que $m>n$. Então $s_{m}-s_{n}=x_{n+1}+x_{n+2}+$ $\ldots+x_{m}=\sum_{j=n+1}^{m} x_{j}, x_{j} \in M\left(A_{j}\right)$ e assim $s_{m}-s_{n} \in M\left(\bigcup_{j=n+1}^{m} A_{j}\right)$.

Para $n_{0}=1$, existem $m_{1}>n_{1}>1 \mathrm{com}\left\|s_{m_{1}}-s_{n_{1}}\right\|>\varepsilon_{0}$. Tomemos $B_{1}=\bigcup_{j=n_{1}+1}^{m_{1}} A_{j} \mathrm{e}$ $y_{1}=s_{m_{1}}-s_{n_{1}}$. Logo $y_{1} \in M\left(B_{1}\right)$ e $\left\|y_{1}\right\|>\varepsilon_{0}$.

Para $n_{0}=m_{1}$, existem $m_{2}>n_{2}>m_{1}$ com $\left\|s_{m_{2}}-s_{n_{2}}\right\|>\varepsilon_{0}$. Tomemos $B_{2}=\bigcup_{j=n_{2}+1}^{m_{2}} A_{j} \mathrm{e}$ $y_{2}=s_{m_{2}}-s_{n_{2}}$. Logo $y_{2} \in M\left(B_{2}\right)$ e $\left\|y_{2}\right\|>\varepsilon_{0}$.

Genericamente, para $n_{0}=m_{k-1}$ sejam $m_{k}>n_{k}>m_{k-1}$ com $\left\|s_{m_{k}}-s_{n_{k}}\right\|>\varepsilon_{0}$. Tomando $B_{k}=\bigcup_{j=n_{k}+1}^{m_{k}} A_{j}$ e $y_{k}=s_{m_{k}}-s_{n_{k}}$, temos $y_{k} \in M\left(B_{k}\right)$ e $\left\|y_{k}\right\|>\varepsilon_{0}$. 
Assim, $\left\{B_{k}\right\}_{k \in \mathbb{N}}$ é uma seqüência disjunta em $\Sigma$, e a seqüência $\left\{y_{k}\right\}$, com $y_{k} \in M\left(B_{k}\right)$, é tal que $\left\|y_{k}\right\|>\varepsilon_{0}$, donde $\lim _{n \rightarrow \infty}\left\|y_{k}\right\| \neq 0$.

Proposição 2.17 Seja $M: \Sigma \longrightarrow P_{f c}(X)$ uma multimedida tal que $M(\Sigma)$ seja um subconjunto de $X$ relativamente fraco-compacto. Então $M$ é fortemente aditiva.

\section{prova:}

Vamos provar inicialmente que dados uma seqüência disjunta $\left\{A_{n}\right\}_{n \in \mathbb{N}}$ em $\Sigma$ e $x_{n}$ em $M\left(A_{n}\right)$ para cada $n \in \mathbb{N}$, a série $\sum_{n \in \mathbb{N}} x^{*}\left(x_{n}\right)$ é convergente para todo $x^{*} \in X^{*}$.

Suponhamos, por absurdo, que exista $x_{1}{ }^{*} \in X^{*}$ tal que a série $\sum_{n \in \mathbb{N}} x_{1}{ }^{*}\left(x_{n}\right)$ seja divergente. Sem perda de generalidade podemos admitir que a série de termos positivos de $\sum_{n \in \mathbb{N}} x_{1}{ }^{*}\left(x_{n}\right)$ é divergente.

Sejam então $p_{i}=x_{1}^{*}\left(x_{n_{i}}\right)>0, i \in \mathbb{N}$ e $x_{1}^{*}\left(x_{n}\right) \leq 0$ se $n \in \mathbb{N}-\left\{n_{i}: i \in \mathbb{N}\right\}$.

Como $M$ é multimedida, $M\left(\bigcup_{n=1}^{k} B_{n}\right)=\sum_{n=1}^{k} M\left(B_{n}\right)$ para quaisquer $B_{1}, B_{2}, \ldots B_{k} \in \Sigma$ dois a dois disjuntos e assim toda subsérie de $\sum_{n \in \mathbb{N}} x_{n}$ tem somas parciais em $M(\Sigma)$, que é relativamente fraco-compacto.

Como $\overline{M(\Sigma)}{ }^{\omega}$ é $\omega$-compacto, temos pelo teorema 1.9 , a seqüência das somas parciais de $\sum_{i \in \mathbb{N}} x_{n_{i}}$ tem subseqüência $\omega$-convergente em $\overline{M(\Sigma)}{ }^{\omega}$, ou seja, sendo $s_{j}=\sum_{i=1}^{j} x_{n_{i}}$, existe $s_{j_{t}}=\sum_{i=1}^{j_{t}} x_{n_{i}} \omega$-convergente para $x_{0} \in X$. Entào $\sum_{i=1}^{j_{t}} x_{1}^{*}\left(x_{n_{i}}\right) \stackrel{t \rightarrow \infty}{\longrightarrow} x_{1}^{*}\left(x_{0}\right)$ e como $p_{i}>0$, isso significa que a série $\sum_{i} p_{i}$ converge para $x_{1}^{*}\left(x_{0}\right)$, o que contradiz a suposição de que $\sum p_{i}$ é divergente.

Portanto, para todo $x^{*} \in X^{*}, \sum_{n \in \mathbb{N}} x^{*}\left(x_{n}\right)$ converge.

Agora vamos mostrar que para toda subsérie $\sum_{k} x_{n_{k}}$ existe $x_{0} \in X$ tal que $\sum_{k \geq 1} x^{*}\left(x_{n_{k}}\right)$ converge para $x^{*}\left(x_{0}\right)$, para todo $x^{*} \in X^{*}$.

Seja $s_{j}=\sum_{k=1}^{j} x_{n_{k}}$. Sabemos que $\sum_{k \in \mathbb{N}} x^{*}\left(x_{n_{k}}\right)$ converge, para todo $x^{*} \in X^{*}$.

De novo, pelo teorema 1.9 , temos que existem $\left\{s_{j_{r}}\right\}$ e $x_{0} \in X$ tais que

$$
x^{*}\left(s_{j_{r}}\right)=x^{*}\left(\sum_{k=1}^{j_{r}} x_{n_{k}}\right) \longrightarrow x^{*}\left(x_{0}\right),
$$

para todo $x^{*} \in X^{*}$.

Por (I) e pela convergência temos a seqüência das somas parciais de $\sum x^{\star}\left(x_{n_{k}}\right)$ convergente, com subseqüência convergente para $x^{*}\left(x_{0}\right)$. Logo, $\left\{x^{*}\left(s_{j}\right)\right\}_{j \in \mathbb{N}}$ converge para $x^{*}\left(x_{0}\right)$. 
Podemos agora aplicar o teorema 1.14, concluindo que toda subsérie de $\sum x_{n}$ é convergente, ou seja, $\sum x_{n}$ é incondicionalmente convergente.

Portanto, $M$ é fortemente aditiva.

Proposição 2.18 Consideremos $M: \Sigma \longrightarrow P_{f c}(X)$ uma multimedida fortemente aditiva tal que a aplicação $A \in \Sigma \mapsto \sigma\left(x^{*}, M(A)\right) \in \mathbb{R} \cup\{\infty\}$ seja uma medida $\sigma$-aditiva para cada $x^{*} \in X^{*}$. Então $M$ é $\sigma$-aditiva.

\section{prova:}

Suponhamos que não. Então existem uma seqüência disjunta $\left\{A_{n}\right\}$ em $\Sigma \operatorname{com} \underset{n \in \mathbb{N}}{\bigcup} A_{n}$ em $\Sigma$ e $x_{n} \in M\left(A_{n}\right), n \in \mathbb{N}$ tais que $\sum_{n} x_{n}$ é convergente para $y \in X$, pois $M$ é fortemente aditiva, mas $y=\sum_{n} x_{n} \notin M\left(\bigcup_{n} A_{n}\right)$.

Como $\{y\}$ é um conjunto convexo e compacto e $M\left(\cup_{n} A_{n}\right)$ é fechado e convexo podemos aplicar o teorema 1.6 e existem constantes reais $c$ e $a$ e $x^{*} \in X^{*}$ tais que $x^{*}(y)<c<a<x^{*}(z)$, para qualquer $z \in M\left(\bigcup_{n} A_{n}\right)$.

Seja $y^{*}=-x^{*}$. Assim $y^{*}(z)<-c<y^{*}(y)$ para todo $z \in M\left(\underset{n}{\cup} A_{n}\right)$. Mas $y^{*}(y)=$ $y^{*}\left(\sum_{n} x_{n}\right)=\sum_{n} y^{*}\left(x_{n}\right) \leq \sum_{n} \sigma\left(y^{*}, M\left(A_{n}\right)\right)$, donde $y^{*}(z)<-c<\sum_{n} \sigma\left(y^{*}, M\left(A_{n}\right)\right)$, para qualquer $z \in M\left(\bigcup_{n} A_{n}\right)$. Logo $\sigma\left(y^{*}, M\left(\cup_{n} A_{n}\right)\right)=\sup _{z \in M\left(\cup_{n} A_{n}\right)} y^{*}(z) \leq-c<\sum_{n} \sigma\left(y^{*}, M\left(A_{n}\right)\right)$, o que contradiz a hipótese. Portanto, $M$ é $\sigma$-aditiva.

Lembramos que dados dois conjuntos $K$ e $L$ de um espaço métrico $M$ a distância de Hausdorff entre eles é dada por:

$$
h(K, L)=\max \left\{\sup _{x \in K} d(x, L), \sup _{x \in L} d(x, K)\right\},
$$

onde $d(x, K)=\inf \{d(x, y), y \in K\}$.

Lema 2.19 Seja $M: \Sigma \longrightarrow P_{0}(X)$ multimedida fortemente aditiva.Então:

(i) $\lim _{n \rightarrow \infty} h\left(\{0\}, \sum_{k=n}^{\infty} M\left(A_{k}\right)\right)=0$, para qualquer seqüência disjunta $\left\{A_{n}\right\}$ em $\Sigma$.

Se $M$ tiver valores em $P_{l f c}(X)$

(ii) $M(\Sigma)$ é um subconjunto limitado de $X$.

(iii) Se $\sigma\left(x^{*}, M(\cdot)\right)$ for $\sigma$-aditiva para cada $x^{*} \in X^{*}$ então a coleçào $\left\{\sigma\left(x^{*}, M(\cdot)\right) / x^{*} \in X^{*} \mathrm{e}\right.$ $\left.\left\|x^{*}\right\| \leq 1\right\}$ é uma família de medidas uniformemente $\sigma$-aditiva. 
prova:

(i) Suponhamos, por absurdo, que exista uma seqüência disjunta $\left\{A_{n}\right\}_{n \in \mathbb{N}}$ em $\Sigma$ tal que $\lim _{n \longrightarrow \infty} h\left(\{0\}, \sum_{k=n}^{\infty} M\left(A_{k}\right)\right) \neq 0$. Então, existem $\varepsilon>0$ e uma subseqüência $\left\{n_{i}\right\}_{i \in \mathbb{N}}$ de $\mathbb{N}$ tais que $h\left(\{0\}, \sum_{k=n_{i}}^{\infty} M\left(A_{k}\right)\right)>\varepsilon$, ou seja, $\sup _{x \in \sum_{k=n_{i}}^{\infty} M\left(A_{k}\right)}\|x\|>\varepsilon$, para todo $i \in \mathbb{N}$.

Dado $k_{1}=n_{1}$, escolhemos $m_{1} \in\left\{n_{i}\right\}_{i \in \mathbb{N}}$ tal que $m_{1}>k_{1}$ e $x_{1} \in \sum_{j=k_{1}}^{m_{1}} M\left(A_{j}\right) \operatorname{com}\left\|x_{1}\right\|>\frac{\varepsilon}{2}$.

Tomando $k_{2}>m_{1}$ e $k_{2} \in\left\{n_{i}\right\}_{i \in \mathbb{N}}$, escolhemos $m_{2} \in\left\{n_{i}\right\}_{i \in \mathbb{N}}$ tal que $m_{2}>k_{2}$ e $x_{2} \in \sum_{j=k_{2}}^{m_{2}} M\left(A_{k}\right) \operatorname{com}\left\|x_{2}\right\|>\frac{\varepsilon}{2}$.

E assim sucessivamente, para cada $r$ podemos construir $x_{r} \in M\left(B_{r}\right)$, onde $B_{r}=\bigcup_{k=n_{r}}^{m_{r}} A_{k}$, $\operatorname{com}\left\|x_{r}\right\|>\frac{\varepsilon}{2}$.

Então $\left\{B_{r}\right\}$ é uma seqüência disjunta em $\Sigma$ com $x_{r} \in M\left(B_{r}\right)$ e $\lim _{r \rightarrow \infty}\left\|x_{r}\right\| \neq 0$, e isso contradiz a hipótese de $M$ ser fortemente aditiva.

(ii) Observemos inicialmente que para todo $A \in \Sigma$ temos $h(\{0\}, M(A))=\sup _{x \in M(A)}\|x\|<\infty$, pois $M(A)$ é limitado.

Suponhamos agora que $M(\Sigma)$ não seja limitado.

Então existe um conjunto $A_{1} \in \Sigma \operatorname{com} h\left(\{0\}, M\left(A_{1}\right)\right)>1$. Como observamos acima, $h\left(\{0\}, M\left(A_{1}\right)\right)<\infty$ logo existe um conjunto $C_{2} \in \Sigma \operatorname{com} h\left(\{0\}, M\left(C_{2}\right)\right)>2 . h\left(\{0\}, M\left(A_{1}\right)\right)$.

Sendo $A_{2}=A_{1} \cup C_{2}^{\prime}$, vale que $h\left(\{0\}, M\left(A_{2}\right)\right)>2 . h\left(\{0\}, M\left(A_{1}\right)\right)$ e $h\left(\{0\}, M\left(A_{2}\right)\right)<\infty$.

Do mesmo modo, existe $C_{3} \in \Sigma \operatorname{com} h\left(\{0\}, M\left(C_{3}\right)\right)>2 . h\left(\{0\}, M\left(A_{2}\right)\right)$.

Tomando $A_{3}=A_{2} \cup C_{3}$, temos $h\left(\{0\}, M\left(A_{3}\right)\right)>2 . h\left(\{0\}, M\left(A_{2}\right)\right)$.

Assim sucessivamente, construímos $\left\{A_{n}\right\}$ em $\Sigma \operatorname{com} h\left(\{0\}, M\left(A_{1}\right)\right)>1, A_{n} \subset A_{n+1}$ e $h\left(\{0\}, M\left(A_{n+1}\right)\right)>2 . h\left(\{0\}, M\left(A_{n}\right)\right)$, para todo $n \in \mathbb{N}$. $n \in \mathbb{N}$.

Consideremos $\left\{B_{n}\right\}$, seqüência disjunta em $\Sigma$, dada por $B_{1}=A_{1}, B_{n+1}=A_{n+1} \cap A_{n}^{c}$, para

Como $A_{n} \cap B_{n+1}=\emptyset, A_{n} \cup B_{n+1}=A_{n+1}$ e $M$ é finitamente aditiva temos,

$$
M\left(A_{n+1}\right)=M\left(A_{n}\right)+M\left(B_{n+1}\right) \text { para todo } n \in \mathbb{N} .
$$

Seja $y_{1} \in M\left(B_{1}\right)=M\left(A_{1}\right)$ com $\left\|y_{1}\right\|>1$.

Escolhendo $x_{2} \in M\left(A_{2}\right)$ com $\left\|x_{2}\right\|>2 . h\left(\{0\}, M\left(A_{1}\right)\right)$, existem $z_{1} \in M\left(A_{1}\right)$ e $y_{2} \in M\left(B_{2}\right)$ tais que $x_{2}=z_{1}+y_{2}$. Daí $\left\|y_{2}\right\|=\left\|x_{2}-z_{1}\right\| \geq\left\|x_{2}\right\|-\left\|z_{1}\right\|>h\left(\{0\}, M\left(A_{1}\right)\right)>1$. 
Tomando $x_{3} \in M\left(A_{3}\right)$ com $\left\|x_{3}\right\|>2 h\left(\{0\}, M\left(A_{2}\right)\right)$, também $x_{3}=z_{2}+y_{3}$, com $z_{2} \in M\left(A_{2}\right)$ e $y_{3} \in M\left(B_{3}\right)$. Novamente, $\left\|y_{3}\right\| \geq\left\|x_{3}\right\|-\left\|z_{2}\right\|>h\left(\{0\}, M\left(A_{2}\right)\right)>h\left(\{0\}, M\left(A_{1}\right)\right)>1$.

Deste modo construímos uma seqüência $\left\{y_{n}\right\}$ tal que para todo $n \in \mathbb{N}, y_{n} \in M\left(B_{n}\right)$ e $\left\|y_{n}\right\|>1$, e portanto $\sum_{n} y_{n}$ não pode ser convergente.

As seqüências $\left\{B_{n}\right\}_{n \in \mathbb{N}}$ em $\Sigma$ e $\left\{y_{n}\right\}$ em $X$ contradizem o fato de $M$ ser fortemente aditiva.

Logo, $M(\Sigma)$ é limitado.

(iii) Pela proposicao 2.18 sabemos que $M$ é $\sigma$-aditiva e portanto, pela proposição 2.13 , é uma multimedida fraca.

Então, dados $x^{*} \in X^{*}$ e uma seqüência disjunta $\left\{A_{n}\right\} \operatorname{com} \bigcup_{n} A_{n} \in \Sigma$, para cada $n \in \mathbb{N}$ vale

$$
\begin{array}{r}
\left|\sum_{k=n}^{\infty} \sigma\left(x^{*}, M\left(A_{k}\right)\right)\right|=\left|\sigma\left(x^{*}, M\left(\bigcup_{k=n}^{\infty} A_{k}\right)\right)\right|=\left|\sup _{x \in \sum_{k=n}^{\infty} M\left(A_{k}\right)} x^{*}(x)\right| \\
\leq \sup _{x \in \sum_{k=n}^{\infty} M\left(A_{k}\right)}\left|x^{*}(x)\right| \leq\left\|x^{*}\right\| \sup _{x \in \sum_{k=n}^{\infty} M\left(A_{k}\right)}\|x\| . \\
\operatorname{Logo}, \lim _{n \longrightarrow \infty} \sup _{\left\|x^{*}\right\| \leq 1}\left|\sum_{k=n}^{\infty} \sigma\left(x^{*}, M\left(A_{k}\right)\right)\right| \leq \lim _{n \rightarrow \infty} \sup _{x \in \sum_{k=n}^{\infty} M\left(A_{k}\right)}\|x\| \stackrel{(\mathrm{i})}{=} 0 .
\end{array}
$$
aditiva.

Assim $\left\{\sigma\left(x^{*}, M(\cdot)\right) / x^{*} \in X^{*}\right.$ e $\left.\left\|x^{*}\right\| \leq 1\right\}$ é uma família de medidas uniformemente $\sigma$ -

Definição 2.20 Sejam $\mu: \Sigma \rightarrow \mathbb{R}_{+}$uma medida e $M: \Sigma \rightarrow P_{0}(X)$ uma multimedida. Se $\lim _{\mu(E) \rightarrow 0} h(\{0\}, M(E))=0$ então $M$ é chamada contínua com respeito a $\mu$. Denotaremos por $M \ll \mu$.

Proposição 2.21 Se $M: \Sigma \longrightarrow P_{l f c}(X)$ é uma multimedida fortemente aditiva tal que para todo $x^{*} \in X^{*}$ a aplicação $\sigma\left(x^{*}, M(\cdot)\right): \Sigma \longrightarrow \mathbb{R} \cup\{\infty\}$ é uma medida $\sigma$-aditiva, então existe uma medida real $\sigma$-aditiva não negativa $\nu$ sobre $\Sigma_{1}$, a $\sigma$-álgebra gerada por $\Sigma$, tal que $\left.M \ll \nu\right|_{\Sigma}$.

\section{prova:}

Pelo Lema 2.19-(iii) temos que a coleção $\left\{\sigma\left(x^{*}, M(\cdot)\right): x^{*} \in X^{*},\left\|x^{*}\right\| \leq 1\right\}$ é uma família de medidas uniformemente $\sigma$-aditiva sobre $\Sigma$.

Como $\left|\sigma\left(x^{*}, M(A)\right)\right| \leq\left\|x^{*}\right\| \sup _{x \in M(\Sigma)}\|x\|$ para cada $x^{*} \in X^{*}$ e $M(\Sigma)$ é limitado pelo lema 2.19 (ii), $\sigma\left(x^{*}, M(\cdot)\right)$ é real e limitada, para cada $x^{*} \in X^{*}$. 
Aplicando o teorema de extensão de Hahn ([3],p136), para cada $x^{*} \in X^{*},\left\|x^{*}\right\| \leq 1$, existe uma única medida $\sigma_{x^{*}}(\cdot)$ que estende $\sigma\left(x^{*} . M(\cdot)\right)$ e também é $\sigma$-aditiva sobre $\Sigma_{1}$, a $\sigma$-álgebra gerada por $\Sigma$.

Logo, por ([4],lema 1,p.26), a família $\left\{\sigma_{x^{*}}(\cdot): x^{*} \in X^{*},\left\|x^{*}\right\| \leq 1\right\}$ é uniformemente $\sigma$ aditiva sobre $\Sigma_{1}$, e sendo limitada, por ([4],teorema 4,p.11) existe uma medida real $\nu$, não negativa, sobre $\Sigma_{1}$ tal que $\lim _{\nu(E) \longrightarrow 0} \sup _{\left\|x^{*}\right\| \leq 1}\left|\sigma_{x^{*}}(E)\right|=0$.

Quando $E \in \Sigma$,

$$
\lim _{\nu(E) \rightarrow 0} \sup _{\left\|x^{*}\right\| \leq 1}\left|\sigma\left(x^{*}, M(E)\right)\right|=\lim _{\nu(E) \rightarrow 0} \sup _{\left\|x^{*}\right\| \leq 1}\left|\sigma_{x^{*}}(E)\right|=0 .
$$

Assim temos que

$$
\lim _{\nu(E) \rightarrow 0} \sup _{\left\|x^{*}\right\| \leq 1}\left|\sup _{z \in M(E)} x^{*}(z)\right|=0 .
$$

Pelo teorema 1.7 , se $x \in M(E)$ e $x \neq 0$ existe $y^{*} \in X^{*}$ tal que $y^{*}(x)=\|x\|$ e $\left\|y^{*}\right\|=1$, donde

$$
0<\|x\|=y^{*}(x) \leq\left|\sup _{z \in M(E)} y^{*}(z)\right| \leq \sup _{\left\|x^{*}\right\| \leq 1}\left|\sup _{z \in M(E)} x^{*}(z)\right| \text {. }
$$

Portanto vale que

$$
0 \leq \lim _{\nu(E) \rightarrow 0} \sup _{x \in M(E)}\|x\| \leq \lim _{\nu(E) \rightarrow 0} \sup _{\left\|x^{*}\right\| \leq 1}\left|\sup _{z \in M(E)} x^{*}(z)\right| \stackrel{(\text { I })}{=} 0 .
$$

Logo, $\lim _{\nu(E) \longrightarrow 0} h(\{0\}, M(E))=0$, ou seja, $\left.\mathrm{M} \ll \nu\right|_{\Sigma}$.

Proposição 2.22 Sejam $\Sigma$ uma $\sigma$-álgebra e $M: \Sigma \longrightarrow P_{w k c}(X)$ uma multimedida fraca. Então $M(\Xi)$ é relativamente $\omega$-compacto e $M$ é $\sigma$-aditiva.

\section{prova:}

Fixado $x^{*} \in X^{*}$, consideremos $\mu_{1}(\cdot)=\sigma\left(x^{*}, M(\cdot)\right)$ e $\mu_{2}(\cdot)=\sigma\left(-x^{*}, M(\cdot)\right)$, que são medidas reais $\sigma$-aditivas na $\sigma$-álgebra $\Sigma$. Pelo teorema de decomposiçạo de Hahn ([3],p 129), para $i=1,2$, existem $P_{i}, N_{i} \in \Sigma$, com $P_{i} \cap N_{i}=\emptyset$ e $P_{i} \cup N_{i}=\Omega$, tais que $\mu_{i}$ é positiva em $P_{i}$ e seus subconjuntos e negativa em $N_{i}$ e seus subconjuntos.

Em particular,

$$
\mu_{i}\left(P_{i}\right) \geq \mu_{i}(C), i=1,2,
$$

para todo $C \in \Sigma$.

Sendo $M\left(P_{i}\right) \omega$-compactos, $i=1,2$, existem $x_{i} \in M\left(P_{i}\right), i=1,2$, com

$$
\mu_{1}\left(P_{1}\right)=\sup _{x \in M\left(P_{1}\right)} x^{*}(x)=x^{*}\left(x_{1}\right)
$$

e 


$$
\mu_{2}\left(P_{2}\right)=\sup _{x \in M\left(P_{2}\right)}-x^{*}(x)=-x^{*}\left(x_{2}\right) .
$$

Dado $x \in M(\Sigma)$, existe $C \in \Sigma$ tal que $x \in M\left(C^{\prime}\right)$, de modo que

$$
x^{*}(x) \leq \mu_{1}(C) \leq x^{*}\left(x_{1}\right) \text { e }-x^{*}(x) \leq \mu_{2}(C) \leq-x^{*}\left(x_{2}\right) .
$$

Então $\left|x^{*}(x)\right| \leq M_{x^{*}}=\max \left\{x^{*}\left(x_{1}\right),-x^{*}\left(x_{2}\right)\right\}$, para todo $x \in M(\Sigma)$.

Pelo princípio da limitação uniforme, $\widehat{M(\Sigma)}$ é limitado em $X^{* *}$, e como $\|\widehat{x}\|=\|x\|$ para $x \in X$, temos $M(\Sigma)$ e também $\overline{M(\Sigma)}{ }^{\omega}$ limitados.

Sendo $\overline{M(\Sigma)}^{\omega}$ limitado e $\omega$-fechado, pelo teorema de James (1.13) resta mostrar que todo $x^{*} \in X^{*}$ assume seu supremo em $\overline{M(\Sigma)} \omega$.

Fixamos novamente $x^{*} \in X^{*}$ e, utilizando (I),(II) e (III), temos que $x^{*}\left(x_{1}\right) \geq \mu_{1}(C) \geq$ $x^{*}(x)$, para todo $C \in \Sigma$ e todo $x \in C$.

Portanto, $x^{*}\left(x_{1}\right) \geq \sup _{x \in M(\Sigma)} x^{*}(x)=\sup _{x \in \overline{M(\Sigma)}} x^{*}(x)$.

Por outro lado, $x^{*}\left(x_{1}\right) \leq \sup _{x \in \overline{M(\Sigma)}} x^{*}(x)$, pois $x_{1} \in M\left(P_{1}\right) \subset \overline{M(\Sigma)}{ }^{\omega}$.

Então $x^{*}\left(x_{1}\right)=\sup _{x \in \overline{M(\Sigma)}} x^{*}(x)$ e $x^{*}$ atinge seu supremo em $\overline{M(\Sigma)}{ }^{\omega}$.

Logo, $\overline{M(\Sigma)}^{\omega}$ é $\omega$-compacto.

Agora vamos mostrar que $M$ é multimedida, isto é, é aditiva. Sejam $A, B \in \Sigma$ disjuntos. Como tanto $M(A \cup B)$ com $M(A)+M(B)$ são $\omega$-compactos e portantó $\omega$-fechados, o resultado segue da proposição $2.13(2)$, pois eles também são convexos.

Portanto, $M$ é multimedida a valores em $P_{w k c}(X)$, com $\overline{M(\Sigma)}{ }^{\omega} \omega$-compacto. Pelas proposições 2.16 e $2.17, M$ é $\sigma$-aditiva.

Definição 2.23 Sejam $M: \Sigma \longrightarrow P_{0}(X)$ uma multimedida, $A \in \Sigma$ e $\Pi$ a coleção de todas as partições finitas disjuntas $\pi$ de $A$. Definimos a variação de $M$ sobre $A$ por $|M|(A)=$ $\sup _{\pi \in \Pi} \sum_{E \in \pi} h(\{0\}, M(E))$.Se $|M|(\Omega)<\infty$ dizemos que M é de variação limitada.

Lema 2.24 Seja $M: \Sigma \longrightarrow P_{0}(X)$ uma multimedida. Para qualquer $A \in \Sigma$ temos $|M|(A) \leq$ $|M|(\Omega)$.

prova:

Sejam $\Pi_{A}$ o conjunto das partiçòes finitas disjuntas $\pi_{A}$ de $A$ e $\Pi$ o conjunto das partições finitas disjuntas $\pi$ de $\Omega$.

Para cada $\pi_{A} \in \Pi_{A}$ temos 


$$
\sum_{F \in \pi_{A \cup\left\{A^{c}\right\}}} h(\{0\}, M(F))=\sum_{F \in \pi_{A}} h(\{0\}, M(F))+h\left(\{0\}, M\left(A^{c}\right)\right) .
$$

Então

$$
\sum_{F \in \pi_{A} \cup\left\{A^{c}\right\}} h(\{0\}, M(F)) \geq \sum_{F \in \pi_{A}} h(\{0\}, M(F)) .
$$

Como $\pi_{A} \cup\left\{A^{c}\right\} \in \Pi$,

$$
|M|(\Omega)=\sup _{\pi \in \Pi} \sum_{E \in \pi} h(\{0\}, M(E)) \geq \sum_{F \in \pi_{A}} h(\{0\}, M(F)) \text { para todo } \pi_{A} \in \Pi_{A} .
$$

Portanto $|M|(\Omega) \geq|M|(A)$.

Proposição 2.25 Seja $M: \Sigma \longrightarrow P_{0}(X)$ uma multimedida de variação limitada. Então sua variação $|M|(\cdot)$ tem valores reais positivos e é monótona e finitamente aditiva.

prova:

Pelo lema anterior, $|M|(A) \leq|M|(\Omega)$ para todo $A \in \Sigma$ e portanto $|M|(\cdot)$ tem valores reais, pois $|M|(\Omega)<\infty$. Também são valores positivos pois $h(K, L) \geq 0$.

Mostremos agora que $|M|$ é monótona.

Sejam $A, B \in \Sigma \operatorname{com} A \subset B, \Pi_{B}$ o conjunto das partições finitas disjuntas $\pi_{B}$ de $B, \Pi_{A}$ o conjunto das partições finitas disjuntas $\pi_{A}$ de $A$.

Para cada $\pi_{A} \in \Pi_{A}$ temos

$$
\begin{aligned}
& \sum_{F \in \pi_{A} \cup\left\{B \cap A^{c}\right\}} h(\{0\}, M(F))=\sum_{F \in \pi_{A}} h(\{0\}, M(F))+h\left(\{0\}, M\left(B \cap A^{c}\right)\right) \\
& \geq c \\
& \sum_{F \in \pi_{A}} h(\{0\}, M(F)),
\end{aligned}
$$

e portanto

$$
\sum_{F \in \pi_{A} \cup\left\{B \cap A^{c}\right\}} h(\{0\}, M(F)) \geq \sum_{F \in \pi_{A}} h(\{0\}, M(F)) .
$$

Como $\pi_{A} \cup\left\{B \cap A^{c}\right\} \in \Pi_{B}$,

$$
|M|(B)=\sup _{\pi_{B} \in \Pi_{B}} \sum_{F \in \pi_{B}} h(\{0\}, M(F)) \geq \sum_{F \in \pi_{A}} h(\{0\}, M(F))
$$

para todo $\pi_{A} \in \Pi_{A}$ e portanto $|M|(B) \geq|M|(A)$. 
Para provar que $|M|$ é finitamente aditiva. consideremos $A, B \in \Sigma$ disjuntos. Observamos que quaisquer partições finitas $\pi_{A}$ de $A$ e $\pi_{B}$ de $B$ disjuntos temos $\pi_{A} \cup \pi_{B}$ é partição de $A \cup B$, de modo que $\Pi$, o conjunto das partiçòes finitas disjuntas $\pi$ de $A \cup B, \Pi \supset\left\{\pi_{A} \cup \pi_{B}: \pi_{A} \in\right.$ $\left.\Pi_{A}, \pi_{B} \in \Pi_{B}\right\}$.

Dado $\varepsilon>0$, existem $\pi_{A}^{\varepsilon} \in \Pi_{A}$ e $\pi_{B}^{\varepsilon} \in \Pi_{B}$ tais que $\sum_{F \in \pi_{A}^{\varepsilon}} h(\{0\}, M(F))>|M|(A)-\frac{\varepsilon}{2}$ e $\sum_{F \in \pi_{B}^{\varepsilon}} h(\{0\}, M(F))>|M|(B)-\frac{\varepsilon}{2}$.

Assim

$$
\begin{aligned}
|M|(A \cup B) & =\sup _{\pi \in \Pi} \sum_{F \in \pi} h(\{0\}, M(F)) \\
& \geq \sum_{F \in \pi_{A}^{\varepsilon}} h(\{0\}, M(F))+\sum_{F \in \pi_{B}^{\varepsilon}} h(\{0\}, M(F)) \\
& >|M|(A)+|M|(B)-\varepsilon .
\end{aligned}
$$

Fazendo $\varepsilon$ tender a 0 , temos

$$
|M|(A \cup B) \geq|M|(A)+|M|(B) .
$$

Por outro lado,

$$
\begin{aligned}
|M|(A \cup B) & =\sup _{\pi \in \Pi_{A \cup B}} \sum_{F \in \pi} h(\{0\}, M(F)) \\
& =\sup _{\pi \in \Pi_{A \cup B}}\left(\sum_{F \in \pi} h(\{0\}, M(F \cap A))+\sum_{F \in \pi} h(\{0\}, M(F \cap B))\right) \\
& \leq \sup _{\pi \in \Pi_{A \cup B}}\left(\sum _ { F \in \pi } h \left(\{0\}, M(\underbrace{F \cap A)}_{\in \pi_{A}})+\left(\sup _{\pi \in \Pi_{A \cup B}} \sum_{F \in \pi} h\left(\{0\}, M(\underbrace{F \cap B}_{\in \pi_{B}})\right)\right.\right.\right. \\
& \leq \sup _{\pi \in \Pi_{A}}\left(\sum_{F \in \pi} h(\{0\}, M(A))\right)+\left(\sup _{\pi \in \Pi_{B}} \sum_{F \in \pi} h(\{0\}, M(B))\right) \\
& \leq|M|(A)+|M|(B) .
\end{aligned}
$$

Temos que

$$
|M|(A \cup B) \leq|M|(A)+|M|(B) .
$$

Por (I) e (II) temos $|M|(A \cup B)=|M|(A)+|M|(B)$.

Proposição 2.26 Seja $M: \Sigma \longrightarrow P_{0}(X)$ uma multimedida forte de variação limitada. Então $|M|: \Sigma \longrightarrow \mathbb{R}$ é medida $\sigma$-aditiva.

\section{prova:} anterior.

Já sabemos que $|M|$ é medida finitamente aditiva e a valores reais positivos pela proposição

Seja $\left\{A_{n}\right\}_{n \geq 1}$ seqüência disjunta em $\Sigma$ com $\bigcup_{n} A_{n} \in \Sigma$. Novamente pela proposição 2.25 temos $|M|$ monótona e como $\bigcup_{n=1}^{k} A_{n} \subset \bigcup_{n=1}^{\infty} A_{n}$, vale que 


$$
|M|\left(\bigcup_{n=1}^{k} A_{n}\right)=\sum_{n=1}^{k}|M|\left(A_{n}\right) \leq|M|\left(\bigcup_{n=1}^{\infty} A_{n}\right) .
$$

Fazendo $k$ tender a $\infty$ em (I),

$$
\sum_{n=1}^{\infty}|M|\left(A_{n}\right) \leq|M|\left(\bigcup_{n=1}^{\infty} A_{n}\right) .
$$

Para a outra desigualdade, temos $|M|\left(\bigcup_{n=1}^{\infty} A_{n}\right)=\sup _{\pi \in \Pi} \sum_{F \in \pi} h(\{0\}, M(F))$, onde $\Pi$ é o conjunto das partições finitas disjuntas $\pi$ de $\bigcup_{n=1}^{\infty} A_{n}$.

Sendo $F \subset \underset{n \in \mathbb{N}}{\cup} A_{n}$ podemos escrever $F=F \cap\left(\cup_{n \in \mathbb{N}} A_{n}\right)=\cup_{n \in \mathbb{N}}\left(F \cap A_{n}\right)$ e então

$$
\begin{aligned}
& |M|\left(\bigcup_{n=1}^{\infty} A_{n}\right) \quad=\quad \sup _{\pi \in \Pi} \sum_{F \in \pi} h\left(\{0\}, M\left(\bigcup_{n=1}^{\infty}\left(F \cap A_{n}\right)\right)\right) \\
& M \sigma \text {-aditiva } \sup _{\pi \in \Pi} \sum_{F \in \pi} h\left(\{0\}, \sum_{n=1}^{\infty} M\left(F \cap A_{n}\right)\right) .
\end{aligned}
$$

Para cada $\pi \in \Pi$ e cada $F \in \pi$ temos,

$$
\begin{aligned}
h\left(\{0\}, \sum_{n=1}^{\infty} M\left(F \cap A_{n}\right)\right. & =\sup _{x \in \sum_{n=1}^{\infty} M\left(F \cap A_{n}\right)}\|x\| \\
& =\sup _{x_{n} \in M\left(A_{n} \cap F\right)}\left\|\sum_{n=1}^{\infty} x_{n}\right\| \\
& \leq \sup _{x_{n} \in M\left(A_{n} \cap F\right)} \sum_{n=1}^{\infty}\left\|x_{n}\right\| \\
& \leq \sum_{n=1}^{\infty} \sup _{x_{n} \in M\left(A_{n} \cap F\right)}\left\|x_{n}\right\| .
\end{aligned}
$$

Logo $h\left(\{0\}, \sum_{n=1}^{\infty} M\left(F \cap A_{n}\right)\right) \leq \sum_{n=1}^{\infty} h\left(\{0\}, M\left(F \cap A_{n}\right)\right)$ e podemos concluir que

$\sum_{F \in \pi} h\left(\{0\}, \sum_{n=1}^{\infty} M\left(F \cap A_{n}\right)\right) \leq \sum_{n \in \mathbb{N}} \sum_{F \in \pi} h\left(\{0\}, M\left(F \cap A_{n}\right)\right) \leq \sum_{n \in \mathbb{N}}|M|\left(A_{n}\right)$

Voltando em (III), temos

$$
|M|\left(\bigcup_{n=1}^{\infty} A_{n}\right) \leq \sum_{n=1}^{\infty}|M|\left(A_{n}\right) .
$$

Por (II) e (IV) temos que $|M|$ é $\sigma$-aditiva.

\subsection{O conjunto $\mathrm{S}_{\mathrm{M}}$ e suas propriedades}

Lembramos que o espaço de todas as medidas vetoriais de $\Sigma$ em $X$ é denotado por $f a(\Sigma . X)$.

Definição 2.27 Seja $M: \Sigma \longrightarrow P_{0}(X)$ uma multimedida. Uma medida $m \in f a(\Sigma, X)$ é um 
seletor de $M$ se $m(A) \in M(A)$, para qualquer $A \in \Sigma$. O conjunto de todos os seletores de $M$ será denotado por $S_{M}$.

Definição 2.28 Um subconjunto $\mathcal{M}$ de $f a(\Sigma, X)$ é decomponível se para $m_{1}, m_{2}$ em $\mathcal{M}$ e todo $A \in \Sigma$ a medida $m: \Sigma \longrightarrow X$ dada por $m(B)=m_{1}(B \cap A)+m_{2}\left(B \cap A^{c}\right)$, para $B \in \Sigma$, pertence a $\mathcal{M}$. Denota-se $m=\chi_{A} m_{1}+\chi_{A^{c}} m_{2}$

Observação 2.29 Para toda multimedida $M: \Sigma \longrightarrow P_{0}(X)$ o subconjunto $S_{M}$ é decomponível pois sabemos que $m \in f a(\Sigma, X)$ e para todo $B \in \Sigma$ temos

$$
\begin{aligned}
& m(B)=m_{1}(B \cap A)+m_{2}\left(B \cap A^{c}\right) \in M(B \cap A)+M\left(B \cap A^{c}\right) \stackrel{\text { multim. }}{=} M\left((B \cap A) \cup\left(B \cap A^{c}\right)\right) . \\
& \quad \text { Logo } m(B) \in M(B) .
\end{aligned}
$$

Teorema 2.30 Seja $M: \Sigma \longrightarrow P_{w k c}(X)$ uma multimedida. Então $S_{M} \neq \emptyset$.

\section{prova:}

Pelo teorema de Zermelo da boa ordem ([7],p8) podemos bem ordenar $X^{*}$, ou seja, definir uma ordem total $\leq$ em $X^{*}$ tal que todo $D \subset X^{*}$ nào vazio tem menor elemento.

Seguindo ([1],p157), definiremos uma relação $\prec$ em $X$ utilizando essa boa ordem de $X^{*}$, que mostraremos ser uma relação de ordem total (é chamada ordem lexicográfica).

Para $x, y \in X$ dizemos que $x \prec y$ se e só se $x=y$ ou, se $x \neq y, x_{0}^{*}(x)<x_{0}^{*}(y)$, onde $x_{0}^{*}=\min \left\{x^{*} \in X^{*}: x^{*}(x) \neq x^{*}(y)\right\}$. Observamos que se $x \neq y$ pelo teorema 1.6 sempre existe pelo menos um $x^{*} \in X^{*} \operatorname{com} x^{*}(x) \neq x^{*}(y)$, de modo que $x_{0}^{*}$ está bem defindo.

É claro que $x \prec x$, pois $x=x$ e vale a propriedade reflexiva.

Para a propriedade anti-simétrica, sejam $x, y \in X \operatorname{com} x \prec y$ e $y \prec x$. Se $x \neq y$, sendo $x_{0}^{*}=\min \left\{x^{*} \in X^{*}: x^{*}(x) \neq x^{*}(y)\right\}$ temos $x_{0}^{*}(x)<x_{0}^{*}(y)$ e $x_{0}^{*}(y)<x_{0}^{*}(x)$, o que é absurdo. Logo, $x=y$.

Quanto à propriedade transitiva, tomemos $x, y, z \in X$ tais que $x \prec y$ e $y \prec z$. Os casos $x=y, y=z$ ou $x=z$ nos levam trivialmente a $x \prec z$. Suponhamos então $x \neq y, y \neq z$ e $x \neq z$ e consideremos os seguintes subconjuntos não vazios de $X^{*}$ :

$$
\begin{aligned}
& D_{1}=\left\{x^{*} \in X^{*}: x^{*}(x) \neq x^{*}(y)\right\} \\
& D_{2}=\left\{x^{*} \in X^{*}: x^{*}(y) \neq x^{*}(z)\right\} \\
& D_{3}=\left\{x^{*} \in X^{*}: x^{*}(x) \neq x^{*}(z)\right\}
\end{aligned}
$$

Sejam $x_{1}^{*}=\min D_{1}, x_{2}^{*}=\min D_{2}, x_{3}^{*}=\min D_{3}$ e $x_{4}^{*}=\min \left(D_{1} \cup D_{2} \cup D_{3}\right)$. Sabemos então que $x_{1}^{*}(x)<x_{1}^{*}(y), x_{2}^{*}(y)<x_{2}^{*}(z)$ e queremos mostrar que $x_{3}^{*}(x)<x_{3}^{*}(z)$.

Como $x_{4}^{*}=\min \left(D_{1} \cup D_{2} \cup D_{3}\right)$, temos $x_{4}^{*} \in D_{1} \cup D_{2} \cup D_{3}$ e $x_{4}^{*} \leq x_{i}^{*}, 1 \leq i \leq 3$.

Se $x_{4}^{*} \in D_{3}$, temos $x_{4}^{*}=x_{3}^{*}$ e quatro possibilidades:

(i) $x_{4}^{*} \in D_{1} \cap D_{2}$; 
(ii) $x_{4}^{*} \in D_{1} \cap D_{2}^{c}$;

(iii) $x_{4}^{*} \in D_{2} \cap D_{1}^{c}$;

(iv) $x_{4}^{*} \in\left(D_{1} \cup D_{2}\right)^{c}$.

No caso (i) $x_{4}^{*} \geq x_{i}^{*}, 1 \leq i \leq 2$, ou seja $x_{4}^{*}=x_{1}^{*}=x_{2}^{*}$. Então, trivialmente,

$$
x_{3}^{*}(x)=x_{4}^{*}(x)<x_{4}^{*}(y)<x_{4}^{*}(z)=x_{3}^{*}(z) \text { e } x \prec z .
$$

Em (ii), temos $x_{4}^{*} \geq x_{1}^{*}$ e portanto $x_{4}^{*}=x_{1}^{*}$ e ainda $x_{4}^{*}(y)=x_{4}^{*}(z)$, de modo que

$$
x_{3}^{*}(x)=x_{4}^{*}(x)<x_{4}^{*}(y)=x_{4}^{*}(z)=x_{3}^{*}(z) \text { e } x \prec z .
$$

Para (iii), $x_{4}^{*} \geq x_{2}^{*}$ e portanto $x_{4}^{*}=x_{2}^{*}$ e ainda $x_{4}^{*}(y)=x_{4}^{*}(z)$. De novo obtemos

$$
x_{3}^{*}(x)=x_{4}^{*}(x)<x_{4}^{*}(y)=x_{4}^{*}(z)=x_{3}^{*}(z) \text { e } x \prec z .
$$

Finalmente, no caso (iv), $x_{4}^{*}(x)=x_{4}^{*}(y)=x_{4}^{*}(z)$, donde

$$
x_{3}^{*}(x)=x_{4}^{*}(x)=x_{4}^{*}(y)=x_{4}^{*}(z)=x_{3}^{*}(z),
$$

o que é absurdo pois $x_{3}^{*} \in D_{3}$.

Se $x_{4}^{*} \notin D_{3}$, temos $x_{4}^{*}(x)=x_{4}^{*}(z)$ e existem três possibilidades, pois com certeza $x_{4}^{*} \in D_{1} \cup D_{2}$ :

(v) $x_{4}^{*} \in D_{1} \cap D_{2}$;

(vi) $x_{4}^{*} \in D_{1} \cap D_{2}^{c}$;

(vii) $x_{4}^{*} \in D_{2} \cap D_{1}^{c}$

No caso (v), $x_{4}^{*}=x_{1}^{*}=x_{2}^{*}$, de modo que $x_{4}^{*}(x)<x_{4}^{*}(y)<x_{4}^{*}(z)$ o que é absurdo pois $x_{4}^{*}(x)=x_{4}^{*}(z)$.

Em (vi), $x_{4}^{*}=x_{1}^{*}$, e $x_{4}^{*}(y)=x_{4}^{*}(z)$. Então $x_{4}^{*}(x)<x_{4}^{*}(y)=x_{4}^{*}(z)$ o que novamente contradiz $x_{4}^{*}(x)=x_{4}^{*}(z)$.

Para (vii), $x_{4}^{*}=x_{2}^{*}$, e $x_{4}^{*}(x)=x_{4}^{*}(y)$. Então $x_{4}^{*}(x)=x_{4}^{*}(y)<x_{4}^{*}(z)$ de novo contradizendo $x_{4}^{*}(x)=x_{4}^{*}(z)$.

Para mostrar agora que $\prec$ é ordem total, consideremos $x, y \in X$, distintos e seja $x_{0}^{*}=\min \left\{x^{\star} \in X^{*}: x^{*}(x) \neq x^{*}(y)\right\}$. Entào $x_{0}^{*}(x)<x_{0}^{*}(y)$ ou $x_{0}^{*}(y)<x_{0}^{*}(x)$, ou seja, $x \prec y$ ou $y \prec x$. Portanto, se $x, y \in X$, temos $x \prec y$ ou $y \prec x$.

Ainda temos que a relação de ordem $\prec$ é compatível com a adição de $X$, isto é, se $x, y, z \in X$ e $x \prec y$, entào $x+z \prec y+z$. 
De fato, de $x \prec y$ obtemos que ou $x=y$ e neste caso $x+z=y+z$ ou, se $x \neq y$, como $D=\left\{x^{*} \in X^{*}: x^{*}(x) \neq x^{*}(y)\right\}=\left\{x^{*} \in X^{*}: x^{*}(x+z) \neq x^{*}(y+z)\right\}$, sendo $x_{0}^{*}=\min D$, $x_{0}^{*}(x)<x_{0}^{*}(y)$. Então $x_{0}^{*}(x+z)<x_{0}^{*}(y+z)$ e $x+y \prec y+z$.

Seja agora dada uma multimedida $M: \Sigma \longrightarrow P_{w k c}(X)$. Vamos mostrar que $S_{M} \neq \emptyset$ definindo um seletor $m: \Sigma \longrightarrow X$ por $m(A) \in M(A)$ e $m(A)$ é elemento maximal de $M(A)$ em relação à ordem $\prec$, para cada $A \in \Sigma$. A existência de tais elementos maximais é obtida pelo Lema de Zorn.

Dado $A \in \Sigma$, consideremos $C=\left\{x_{\alpha}: \alpha \in I\right\}$ um subconjunto totalmente ordenado de $M(A)$. A ordem total em $X$ induz uma ordem total em $I$ que o torna um conjunto dirigido: $\alpha \leq \beta, \alpha, \beta \in I$ se e só se $x_{\alpha} \prec x_{\beta}$.

Considerando o net $\left\{x_{\alpha}\right\}_{\alpha \in I}$ em $M(A)$ (com essa ordem em $I$ ), ele é um net crescente em $X$, isto é, se $\alpha \leq \beta, x_{\alpha} \prec x_{\beta}$. Como $M(A)$ é $\omega$-compacto, existe um subnet $\left\{x_{\alpha_{\beta}}\right\}_{\beta \in J} \omega$-convergente para $x_{0} \in M(A)$. Mas sendo o net $\left\{x_{\alpha}\right\}_{\alpha \in I}$ crescente, temos então que ele é $\omega$-convergente para $x_{0}$ e $x_{0}$ é um limitante superior de $C$.

Pelo Lema de Zorn ([7],p14), $M(A)$ tem elemento maximal $x_{A}$, isto é, $x_{A} \in M(A)$ e se $y \in M(A) \operatorname{com} x_{A} \prec y$ então $y=x_{A}$.

Definimos $m: \Sigma \longrightarrow X$ por $m(A)=x_{A}, A \in \Sigma$, e vamos mostrar que $m$ é finitamente aditiva, donde seguirá $m \in S_{M}$.

Sejam $A, B \in \Sigma$ disjuntos. Temos que $m(A)+m(B) \in M(A)+M(B)=M(A \cup B)$, e como a ordem $\prec$ é total,

$$
m(A)+m(B) \prec m(A \cup B) .
$$

Por outro lado, como $m(A \cup B) \in M(A)+M(B)$, existem $y \in M(A)$ e $z \in M(B)$ tais que $y+z=m(A \cup B)$. Mas $y \prec m(A)$ e $z \prec m(B)$ pois a ordem $\prec$ é total e daí

$$
y+z \prec m(A)+z \text { e } m(A)+z \prec m(A)+m(B)
$$

porque a ordem $\prec$ é compatível com a adição de $X$.

Pela transitividade, $y+z \prec m(A)+m(B)$, ou

$$
m(A \cup B) \prec m(A)+m(B) .
$$

Por (I) e (II), $m$ é aditiva.

Logo $S_{M} \neq \emptyset$.

Proposição 2.31 Seja $M: \Sigma \longrightarrow P_{0}(X)$ multimedida. Então

(i) se $m \in S_{M}$, então $|m|(A) \leq|M|(A)$, para todo $A \in \Sigma$;

(ii) se $M$ for de variação limitada; $S_{M} \subset b v(\Sigma, X)$;

(iii) se $\Sigma$ for $\sigma$-álgebra e $M$ for multimedida forte, $S_{M} \subset c a(\Sigma, X)$. 


\section{prova:}

(i) Sejam $m \in S_{M}$ e $A \in \Sigma$. Para qualquer $B \in \Sigma$ temos $m(B) \in M(B)$ e portanto $\|m(B)\| \leq \sup _{x \in M(B)}\|x\|=h(\{0\}, M(B))$. Então

$$
\begin{aligned}
|m|(A) & =\sup _{\pi \in \Pi} \sum_{F \in \pi}\|m(F)\| \\
& \leq \sup _{\pi \in \Pi} \sum_{F \in \pi} h(\{0\}, M(F)) \\
& =|M|(A) .
\end{aligned}
$$

(ii) Dado $m \in S_{M}$ da parte (i) temos $|m|(\Omega) \leq|M|(\Omega)$ e se $M$ for de variação limitada obtemos $|m|(\Omega)<+\infty$, ou seja, $m \in b v(\Sigma, X)$.

(iii) Sejam $m \in S_{M}$ e $\left\{A_{n}\right\}_{n \in \mathbb{N}}$ seqüência disjunta em $\Sigma$.

Então

$$
\begin{aligned}
\left\|m\left(\bigcup_{n=1}^{\infty} A_{n}\right)-\sum_{n=1}^{k} m\left(A_{n}\right)\right\| & =\left\|m\left(\bigcup_{n=1}^{\infty} A_{n}\right)-m\left(\bigcup_{n=1}^{k} A_{n}\right)\right\| \\
& =\left\|m\left(\bigcup_{n=k+1}^{\infty} A_{n}\right)\right\| \\
& \leq h\left(\{0\}, M\left(\bigcup_{n=k+1}^{\infty} A_{n}\right)\right) .
\end{aligned}
$$

Como $\Sigma$ é $\sigma$-álgebra e $M \sigma$-aditiva, $M$ é também fortemente aditiva. Podemos então usar o Lema $2.19(\mathrm{i})$, obtendo $h\left(\{0\}, \sum_{n=k+1}^{\infty} M\left(A_{n}\right)\right) \stackrel{k \rightrightarrows \infty}{\longrightarrow} 0$ e portanto $m\left(\bigcup_{n=1}^{\infty} A_{n}\right)=\sum_{n=1}^{\infty} m\left(A_{n}\right)$, ou seja, $m \in c a(\Sigma, X)$.

Definição 2.32 Definimos no conjuntos das funções de $\Sigma$ em $X, \mathcal{F}(\Sigma, X)$, a topologia $\tau$ da convergência pontual fraca da seguinte forma: um net $\left\{m_{\alpha}\right\}_{\alpha \in D}$ de $\mathcal{F}(\Sigma, X)$ é $\tau$-convergente para $m_{0} \in \mathcal{F}(\Sigma, X)$ se para todo $A \in \Sigma,\left\{m_{\alpha}\right\}_{\alpha \in D}$ é $\omega$-convergente para $m_{0}(A)$ em $X$.

Teorema 2.33 Seja $M: \Sigma \longrightarrow P_{w k c}(X)$ uma multimedida. Então $S_{M}$ é $\tau$-compacto.

\section{prova:}

Para qualquer $A \in \Sigma$. o conjunto $H(A)=\left\{m(A) / m \in S_{M}\right\}$ é relativamente fraco-compacto sobre $X$, pois $\overline{H(A)}^{\omega} \subset \overline{M(A)}^{\omega}$ e $M(A)$ é $\omega$-compacto.

Em virtude do teorema 1.15 , para $S_{M}$ ser $\tau$-compacto basta mostrar que $S_{M}$ é pontualmente fechado em $\mathcal{F}(\Sigma, X)$.

Seja $m$ pertencente ao aderente de $S_{M}$ em $\mathcal{F}(\Sigma, X)$ com a topologia $\tau$. Então existem um net $\left\{m_{\alpha}\right\}_{\alpha \in D}$ em $S_{M}$, e uma função $m \in \mathcal{F}(\Sigma, X)$ tais que $m_{\alpha}(E) \stackrel{\omega}{\longrightarrow} m(E)$, para todo $E \in \Sigma$.

Como $m_{\alpha}(E) \in M(E)$ e $M(E)$ é $\omega$-compacto, $m(E) \in M(E)$. Para que $m \in S_{M}$ falta verificar que $m$ é medida. 
Sejam $A, B \in \Sigma$ disjuntos. Sabemos que para $m_{\alpha}$ vale que $m_{\alpha}(A \cup B)=m_{\alpha}(A)+m_{\alpha}(B)$. Por outro lado $m_{\alpha}(E) \stackrel{\omega}{\longrightarrow} m(E)$ para qualquer $E \in \Sigma$.

Assim

$$
m_{\alpha}(A)+m_{\alpha}(B)=m_{\alpha}(A \cup B) \stackrel{\omega}{\longrightarrow} m(A \cup B) .
$$

Como também que

$$
m_{\alpha}(A) \stackrel{\omega}{\longrightarrow} m(A) \text { e } m_{\alpha}(B) \stackrel{\omega}{\longrightarrow} m(B) .
$$

Por (I) e (II) temos $m(A \cup B)=m(A)+m(B)$.

Logo, $m \in S_{M}$, e isto nos garante que $S_{M}$ é pontualmente fechado em $\mathcal{F}(\Sigma, X)$. Portanto $S_{M}$ é $\tau$-compacto.

Lema 2.34 Se $K_{1}, K_{2} \in P_{0}(X)$ e $x \in$ ext $\left(K_{1}+K_{2}\right)$, existem e são únicos os elementos $x_{1} \in K_{1}, x_{2} \in K_{2}$ tais que $x=x_{1}+x_{2}$. Além disso, $x_{1} \in$ ext $K_{1}$ e $x_{2} \in$ ext $K_{2}$.

\section{prova:}

É claro que existem $x_{1} \in K_{1}$ e $x_{2} \in K_{2} \operatorname{com} x=x_{1}+x_{2}$, pois ext $\left(K_{1}+K_{2}\right) \subset K_{1}+K_{2}$.

Para a unicidade, suponhamos que $x_{1}, x_{1}^{\prime} \in K_{1}$ e $x_{2}, x_{2}^{\prime} \in K_{2}$ sejam tais que

$$
x=x_{1}+x_{2}=x_{1}^{\prime}+x_{2}^{\prime} .
$$

Então $x=\frac{1}{2}\left(x_{1}+x_{1}^{\prime}\right)+\frac{1}{2}\left(x_{2}+x_{2}^{\prime}\right)$ e, como $x_{1}+x_{2}^{\prime}, x_{1}^{\prime}+x_{2} \in K_{1}+K_{2}$ e $x \in \operatorname{ext}\left(K_{1}+K_{2}\right)$, concluímos que

$$
x_{1}+x_{2}^{\prime}=x_{1}^{\prime}+x_{2} .
$$

De (I) temos $x_{1}-x_{2}^{\prime}=x_{1}^{\prime}-x_{2}$ e usando (II) obtemos $x_{1}=x_{1}^{\prime}$ e $x_{2}=x_{2}^{\prime}$.

Se $x_{1}$ não fosse extremal de $K_{1}$, existiria $y \in X$ com $x_{1}+y, x_{1}-y \in K_{1}$ ( $x_{1}$ seria ponto médio de algum segmento com extremos em $\left.K_{1}\right)$. Então $x+y=\left(x_{1}+y\right)+x_{2}$ e $x-y=\left(x_{1}-y\right)+x_{2}$ pertenceriam a $K_{1}+K_{2}$ e $x$ não seria extremal de $K_{1}+K_{2}$, contrariando a hipótese. Logo, $x_{1} \in$ ext $K_{1}$.

Analogamente $x_{2} \in$ ext $K_{2}$.

Para $B \in \Sigma$ usaremos a notação $\sum \cap B$ para a álgebra $\{A \cap B: A \in \Sigma\}$.

Proposição 2.35 Se $M: \Sigma \longrightarrow P_{w k c}(X)$ é multimedida, então para todo $B \in \Sigma$ temos $\mathcal{B}=\left\{m(B):\left.m \in S_{M}\right|_{\Sigma \cap B}\right\} \in P_{w k c}(X)$.

\section{prova:}

Temos $\mathcal{B} \neq \emptyset$, pois $S_{M} \neq \emptyset$. 
Sejam $m_{1}, m_{2} \in S_{M} \mid \Sigma \cap B$ e $\lambda \in[0,1]$. Então $m=\lambda m_{1}+(1-\lambda) m_{2}$ é medida vetorial $\sigma$-aditiva e também $m(B)=\lambda m_{1}(B)+(1-\lambda) m_{2}(B) \in \lambda M(B)+(1-\lambda) M(B)=M(B)$, pois $m_{1}(B), m_{2}(B) \in M(B)$ e $M(B)$ é convexo. Assim $\mathcal{B}$ é convexo.

Consideremos agora $\left\{m_{\alpha}(B)\right\}$ em $\mathcal{B}$. Como $\left.S_{M}\right|_{\Sigma \cap B}$ é $\tau$-compacto, existem $\left.m_{0} \in S_{M}\right|_{\Sigma \cap B}$ e $\left\{m_{\alpha_{\beta}}\right\}_{\beta \in J} \operatorname{com} m_{\alpha_{\beta}} \stackrel{\tau}{\longrightarrow} m_{0}$, ou seja, para todo $C \in \Sigma \cap B, m_{\alpha_{\beta}}(C) \stackrel{\omega}{\longrightarrow} m_{0}(C)$. Em particular, $m_{\alpha_{\beta}}(B) \stackrel{\omega}{\longrightarrow} m_{0}(B)$ com $m_{0}(B) \in \mathcal{B}$ e daí concluimos que $\mathcal{B}$ é $\omega$-compacto.

Teorema 2.36 Sejam $M: \Sigma \longrightarrow P_{w k c}(X)$ multimedida e $B \in \Sigma$ fixado. Então, para todo $x \in M(B)$, existe $m \in S_{M} \mid \Sigma_{n} B$ tal que $m(B)=x$.

\section{prova:}

Suponhamos inicialmente que $x \in$ ext $M(B)$. Para todo $C \in \Sigma \cap B$, como $M(B)=$ $M(C)+M\left(B \cap C^{c}\right)$, pelo lema 2.34 existem e são únicos os elementos $x_{C} \in$ ext $M(C)$ e $y_{C} \in \operatorname{ext} M\left(B \cap C^{c}\right)$ tais que $x=x_{C}+y_{C}$.

Definindo $m: \Sigma \cap B \longrightarrow X$ por $m(C)=x_{C}$, vamos verificar que $\left.m \in S_{M}\right|_{\Sigma \cap B}$ e $m(B)=x$.

Para que $\left.m \in S_{M}\right|_{\Sigma \cap B}$, basta mostrar que $m$ é medida vetorial, pois já temos $m(C)=x_{C} \in$ $M(C)$, para todo $C \in \Sigma \cap B$.

Sejam $C_{1}, C_{2} \in \Sigma \cap B$ disjuntos. Como $x_{C_{1} \cup C_{2}} \in M\left(C_{1} \cup C_{2}\right)=M\left(C_{1}\right)+M\left(C_{2}\right)$, de novo pelo lema 2.34 existem (únicos) $z_{C_{1}} \in \operatorname{ext} M\left(C_{1}\right)$ e $z_{C_{2}} \in \operatorname{ext} M\left(C_{2}\right)$, com $x_{C_{1} \cup C_{2}}=z_{C_{1}}+z_{C_{2}}$. Também existe (único) $y_{C_{1} \cup C_{2}} \in \operatorname{ext} M\left(\left(C_{1} \cup C_{2}\right)^{c} \cap B\right)$ com $x=x_{C_{1} \cap C_{2}}+y_{C_{1} \cup C_{2}}$.

Então $z_{C_{1}}+z_{C_{2}}+y_{C_{1} \cup C_{2}}=x$. Como $z_{C_{2}}+y_{C_{1} \cup C_{2}} \in M\left(C_{2}\right)+M\left(\left(C_{1} \cup C_{2}\right)^{c} \cap B\right) \mathrm{e}$

$$
\begin{aligned}
M\left(C_{2}\right)+M\left(\left(C_{1} \cup C_{2}\right)^{c} \cap B\right) & =M\left(C_{2} \cup\left(\left(C_{1} \cup C_{2}\right)^{c} \cap B\right)\right) \\
& =M\left(\left(C_{2} \cup C_{1}^{c}\right) \cap\left(C_{2} \cup C_{2}^{c}\right) \cap\left(C_{2} \cup B\right)\right) \\
& =M\left(C_{1}^{c} \cap B\right),
\end{aligned}
$$

temos $z_{C_{1}}=x_{C_{1}}$, pela unicidade.

Analogamente $z_{C_{2}}=x_{C_{2}}$, de modo que $m\left(C_{1} \cup C_{2}\right)=x_{C_{1} \cup C_{2}}=x_{C_{1}}+x_{C_{2}}=m\left(C_{1}\right)+m\left(C_{2}\right)$.

Suponhamos agora $x \in \operatorname{co}($ ext $M(B))$. Então existem $x_{i} \in \operatorname{ext} M(B)$ e $\lambda_{i}>0,1 \leq i \leq n$, $\operatorname{com} \sum_{i=1}^{n} \lambda_{i}=1$ e $x=\sum_{i=1}^{n} \lambda_{i} x_{i}$.

Pelo que fizemos antes, existem $\left.m_{i} \in S_{M}\right|_{\Sigma \cap B} \operatorname{com} m_{i}(B)=x_{i}, 1 \leq i \leq n$. A medida vetorial $m=\sum_{i=1}^{n} \lambda_{i} m_{i}$ também é seletor de $\left.M\right|_{\Sigma \cap B}$ e vale que $m(B)=\sum_{i=1}^{n} \lambda_{i} x_{i}=x$.

Obtemos assim que

$$
\operatorname{co}(\text { ext } M(B)) \subset\left\{m(B): m \in S_{M} \mid \Sigma \cap B\right\} \subset M(B) .
$$

Lembrando que, por 1.12, $M(B)=\overline{c o}^{\omega}($ ext $M(B))$, pois é convexo e $\omega$-compacto, tomando o fecho na topologia fraca nas inclusões em $(\mathrm{I})$ e usando a proposição anterior $\left(\mathcal{B} \in P_{w k c}(X)\right)$ 
temos finalmente $M(B)=\left\{m(B):\left.m \in S_{M}\right|_{\Sigma \cap B}\right\}$, ou seja, para todo $x \in M(B)$ existe um $\left.m \in S_{M}\right|_{\Sigma \cap B} \operatorname{com} m(B)=x$.

Teorema 2.37 Se $M: \Sigma \longrightarrow P_{w k c}(X)$ é multimedida, então $M(A)=\left\{m(A): m \in S_{M}\right\}$, para todo $A \in \Sigma$.

\section{prova:}

Seja $A \in \Sigma$ e consideremos $\mathcal{A}=\left\{m(A): m \in S_{M}\right\}$.

É claro que $\mathcal{A} \subset M(A)$.

Por outro lado, dado $x \in M(A)$ e tomando $y \in M\left(A^{c}\right)$ arbitrário, pelo teorema 2.35, existem $\left.m_{1} \in S_{M}\right|_{\Sigma \cap A}$ e $\left.m_{2} \in S_{M}\right|_{\Sigma \cap A^{c}}$ tais que $m_{1}(A)=x$ e $m_{2}\left(A^{c}\right)=y$.

Definindo $m: \Sigma \longrightarrow X$ por $m(C)=m_{1}(C \cap A)+m_{2}\left(C \cap A^{c}\right)$, temos que $m$ é medida vetorial, $m(A)=m_{1}(A)=x$ e $m(C) \in M(C)$, pois $m_{1}(C \cap A) \in M(C \cap A), m_{2}\left(C \cap A^{c}\right) \in M\left(C \cap A^{c}\right) \mathrm{e}$ $M$ é aditiva.

Portanto, $x \in \mathcal{A}$. 


\section{Capítulo 3}

\section{Extensão de uma multimedida}

Este capítulo dedica-se ao estudo das condições para que uma multimedida $M$ definida numa álgebra $\Sigma$ de subconjuntos de $\Omega$ possa ser estendida para a $\sigma$-álgebra $\Sigma_{1}$ de subconjuntos de $\Omega$ gerada por $\Sigma$.

Lema 3.1 Sejam $\Sigma$ uma álgebra de subconjuntos de $\Omega$ e $M_{0}: \Sigma \longrightarrow P_{l f c}(X)$ uma multimedida.Consideremos a função $g: X^{*} \times \Sigma \longrightarrow \overline{\mathbb{R}}$ definida por $g\left(x^{*} ; B\right)=\sup _{x \in M_{0}(B)} x^{*}(x)$. Então

(i) para cada $B \in \Sigma$ fixo, $g(\cdot ; B): X^{*} \longrightarrow \overline{\mathbb{R}}$ é uma função convexa real e $\omega^{*}$-semicontínua inferiormente;

(ii) para cada $x^{*} \in X^{*}$ fixo, $g\left(x^{*}, \cdot\right): \Sigma \longrightarrow \mathbb{R}$ é medida (aditiva) real.

\section{prova:}

Observamos inicialmente que $g\left(x^{*} ; B\right) \in \mathbb{R}$ para todo $x^{*} \in X^{*}$ e todo $B \in \Sigma$, pois $M_{0}(B)$ é limitado.

(i) Fixado $B \in \Sigma$ e dados $x^{*}, y^{*} \in X^{*}$ e $0<\lambda<1$, temos $g\left(\lambda x^{*}+(1-\lambda) y^{*} ; B\right)=$ $\sup _{x \in M_{0}(B)}\left(\lambda x^{*}+(1-\lambda) y^{*}\right)(x) \leq \lambda \sup _{x \in M_{0}(B)} x^{*}(x)+(1-\lambda) \sup _{x \in M_{0}(B)} y^{*}(x)$ e portanto $g(\cdot ; B)$ é convexa real.

Agora dados $x_{0}^{*} \in X^{*}$ e $\varepsilon>0$, existe $x_{0} \in M_{0}(B)$ tal que $x_{0}^{*}\left(x_{0}\right)>g\left(x_{0}^{*} ; B\right)-\varepsilon$, ou seja, $-g\left(x_{0}^{*} ; B\right)>-x_{0}^{*}\left(x_{0}\right)-\varepsilon$.

$\operatorname{Assim} g\left(x^{*} ; B\right)-g\left(x_{0}^{*} ; B\right)>\sup _{x \in M_{0}(B)} x^{*}(x)-x_{0}^{*}\left(x_{0}\right)-\varepsilon$, e como $x_{0} \in M_{0}(B)$ temos $g\left(x^{*} ; B\right)-$ $g\left(x_{0}^{*} ; B\right)>x^{*}\left(x_{0}\right)-x_{0}^{*}\left(x_{0}\right)-\varepsilon$.

Escolhendo $V_{x_{0}^{*}}=\left\{x^{*} \in X^{*}:\left|x^{*}\left(x_{0}\right)-x_{0}^{*}\left(x_{0}\right)\right|<\varepsilon\right\}$, então $V_{x_{0}^{*}}^{*}$ é uma vizinhança $\omega^{*}$ de $x_{0}^{*}$ e para $x^{*} \in V_{x_{0}^{*}}$ temos $g\left(x^{*} ; B\right)-g\left(x_{0}^{*} ; B\right)>-2 \varepsilon$. Logo $g(\cdot ; B)$ é $\omega^{*}$-semicontínua inferiormente em $x_{0}^{*}$.

(ii) Segue da proposição $2.13(3)$, pois $M$ é multimedida. 
Lema 3.2 Sejam $\Sigma, M_{0}$ e $g$ como no lema 3.1. Suponhamos que exista uma medida aditiva $\mu$ real não negativa em $\Sigma$ tal que $M_{0} \ll \mu$. Então, para cada $x^{*} \in X^{*}$ fixado,

(i) $\lim _{\mu(E) \longrightarrow 0}\left|g\left(x^{*} ; E\right)\right|=0$, ou seja $g\left(x^{*}, \cdot\right)$ é $\mu$-contínua;

(ii) $g\left(x^{*} ; \cdot\right)$ é uniformemente contínua em $\Sigma$ com respeito a $d: \Sigma \times \Sigma \longrightarrow \mathbb{R}_{+}$dada por $d(A, B)=\mu(A \triangle B)$

e se $\mu$ for $\sigma$-aditiva, temos ainda que

(iii) $g\left(x^{*}, \cdot\right)$ é $\sigma$-aditiva.

\section{prova:}

Seja $x^{*} \in X^{*}$ fixado.

(i) Dado $\varepsilon>0$, existe $\delta_{\varepsilon}>0$ tal que $E \in \Sigma$ e $\mu(E)<\delta_{\varepsilon}$ implicam em $h\left(\{0\}, M_{0}(E)\right)<\varepsilon$, pois $M_{0} \ll \mu$.

Como $\left|g\left(x^{*} ; E\right)\right|=\left|\sup _{x \in M_{0}(E)} x^{*}(x)\right| \leq \sup _{x \in M_{0}(E)}\left|x^{*}(x)\right| \leq\left\|x^{*}\right\| \sup _{x \in M_{0}(E)}\|x\|$, temos

$$
\left|g\left(x^{*} ; E\right)\right| \leq\left\|x^{*}\right\| h\left(\{0\}, M_{0}(E)\right) \leq\left\|x^{*}\right\| \varepsilon
$$

sempre que $E \in \Sigma$ e $\mu(E)<\delta_{\varepsilon}$.

Portanto, $\lim _{\mu(E) \rightarrow 0}\left|g\left(x^{*} ; E\right)\right|=0$ e $g\left(x^{*}, \cdot\right)$ é $\mu$-contínua.

(ii) Vamos mostrar que dado $\varepsilon>0$ existe $\delta_{1}>0$ tal que se $A, B$ em $\Sigma, d(A, B)=$ $\mu(A \triangle B)<\delta_{1}$, vale que $\left|g\left(x^{*} ; A\right)-g\left(x^{*} ; B\right)\right|<\varepsilon\left\|x^{*}\right\|$.

Façamos inicialmente uma estimativa para $\left|g\left(x^{*} ; A\right)-g\left(x^{*} ; B\right)\right|$.

Sabemos que $A \cup B=A \cup\left(B \cap(A \cap B)^{c}\right)=B \cup\left(A \cap(A \cap B)^{c}\right)$ (uniões disjuntas) e como $g\left(x^{*}, \cdot\right)$ é medida real podemos escrever $\left.g\left(x^{*} ; A\right)+g\left(x^{*} ; B \cap(A \cap B)^{c}\right)\right)=g\left(x^{*} ; B\right)+g\left(x^{*} ; A \cap(A \cap B)^{c}\right)$.

Logo

$$
\left|g\left(x^{*} ; A\right)-g\left(x^{*} ; B\right)\right| \leq\left|g\left(x^{*} ; A \cap(A \cap B)^{c}\right)\right|+\left|g\left(x^{*} ; B \cap(A \cap B)^{c}\right)\right| .
$$

Mas $\left|g\left(x^{*}, C\right)\right| \leq \sup _{x \in M_{0}(C)}\left|x^{*}(x)\right| \leq\left\|x^{*}\right\| \sup _{x \in M_{0}(C)}\|x\|=\left\|x^{*}\right\| h\left(\{0\}, M_{0}(C)\right)$, para todo $C \in$ $\Sigma$ e voltando a (I) temos

$$
\left|g\left(x^{*} ; A\right)-g\left(x^{*} ; B\right)\right| \leq\left\|x^{*}\right\|\left\{h\left(\{0\}, M_{0}\left(A \cap(A \cap B)^{c}\right)\right)+h\left(\{0\}, M_{0}\left(B \cap(A \cap B)^{c}\right)\right)\right\} .
$$

Seja dado $\varepsilon>0$. Como $M_{0} \ll \mu$, existe $\delta_{1}>0$ tal que se $E \in \Sigma$ e $\mu(E)<\delta_{1}$ entào $h\left(\{0\}, M_{0}(E)\right)<\frac{\Xi}{2}$. 
Dados $A, B \in \Sigma \operatorname{com} \mu(A \triangle B)<\delta_{1}$, como os conjuntos $\left(A \cap(A \cap B)^{c}\right)$ e $\left(B \cap(A \cap B)^{c}\right)$ são subconjuntos de $A \triangle B$ temos que $\mu\left(A \cap(A \cap B)^{c}\right)<\delta_{1}$ e $\mu\left(B \cap(A \cap B)^{c}\right)<\delta_{1}$.

Utilizando (II) concluimos que se $d(A, B)<\delta_{1}$ então $\left|g\left(x^{*} ; A\right)-g\left(x^{*} ; B\right)\right|<\left\|x^{*}\right\| . \varepsilon$.

Portanto $g\left(x^{*} ; \cdot\right)$ é uniformemente contínua em $\Sigma$.

(iii) Vamos provar que $g\left(x^{*}, \cdot\right)$ é $\sigma$-aditiva, supondo que $\mu$ é $\sigma$-aditiva.

Dada uma seqüência disjunta $\left\{A_{n}\right\}$ em $\Sigma \operatorname{com} \bigcup_{n} A_{n} \in \Sigma$, como $g\left(x^{*}, \cdot\right)$ é finitamente aditiva, temos que $\left|g\left(x^{*}, \bigcup_{n=1}^{\infty} A_{n}\right)-\sum_{n=1}^{r} g\left(x^{*}, A_{n}\right)\right|=\left|g\left(x^{*}, \bigcup_{n=r+1}^{\infty} A_{n}\right)\right|$.

Mas $\mu\left(\bigcup_{n=r+1}^{\infty} A_{n}\right) \stackrel{r \rightarrow \infty}{\longrightarrow} 0$ e então, por (ii) $\left|g\left(x^{*}, \underset{n=r+1}{\bigcup} A_{n}\right)\right|^{r} \longrightarrow \infty 0$.

Logo $g\left(x^{*}, \cdot\right)$ é $\sigma$-aditiva.

Lema 3.3 Sejam $\Sigma, M_{0}, g$ como no lema 3.1 e $\Sigma_{1}$ a $\sigma$-álgebra gerada por $\Sigma$. Suponhamos que exista uma medida real não negativa $\sigma$-aditiva $\mu \mathrm{em} \Sigma_{1}$ tal que $\left.M_{0} \ll \mu\right|_{\Sigma}$ e seja $d: \Sigma_{1} \times \Sigma_{1} \longrightarrow \mathbb{R}$ definida por $d(A, B)=\mu(A \triangle B)$. Então

(i) para cada $x^{*} \in X^{*}$ e para $A \in \Sigma_{1}$, se $\left\{B_{n}\right\}_{n \in \mathbb{N}}$ em $\Sigma$ for tal que $d\left(A, B_{n}\right) \longrightarrow 0$, existe $\lim _{n \longrightarrow \infty} g\left(x^{*} ; B_{n}\right) \in \mathbb{R}$, que independe da seqüência $\left\{B_{n}\right\}$.

Podemos definir $G: X^{*} \times \Sigma_{1} \longrightarrow \mathbb{R}$ por

$G\left(x^{*} ; A\right)=\lim _{n \rightarrow \infty} g\left(x^{*}, B_{n}\right)$ e valem, para cada $x^{*} \in X^{*}$,

(ii) $G\left(x^{*} ; B\right)=g\left(x^{*} ; B\right)$, para todo $B \in \Sigma$;

(iii) $\lim _{\mu(E) \rightarrow 0}\left|G\left(x^{*} ; E\right)\right|=0$;

(iii) $G\left(x^{*} ; \cdot\right)$ é uniformemente contínua em $\Sigma_{1}$ com respeito a $d$;

(iv) $G\left(x^{*} ; \cdot\right)$ é medida real $\sigma$-aditiva.

prova:

(i) Fixamos $x^{*} \in X^{*}$ e seja $A \in \Sigma_{1}$. Sempre existe alguma seqüência $\left\{B_{n}\right\}$, pela proposição 1.36 , tal que $d\left(B_{n}, A\right) \longrightarrow 0$.

Segue que $d\left(B_{n}, B_{m}\right) \stackrel{m, n \rightarrow \infty}{\longrightarrow} 0$. Pelo lema $3.2, g\left(x^{*}, \cdot\right)$ é uniformemente contínua e portanto $\lim _{n, m \rightarrow \infty}\left|g\left(x^{*} ; B_{n}\right)-g\left(x^{*} ; B_{m}\right)\right|=0$. Assim, $\left\{g\left(x^{*} ; B_{n}\right)\right\}_{n \in \mathbb{N}}$ é de Cauchy. Logo existe $\lim _{n} g\left(x^{*} ; B_{n}\right) \in \mathbb{R}$.

Dadas seqüências $\left\{B_{n}\right\},\left\{C_{n}\right\}$ em $\Sigma$ tais que $d\left(B_{n}, A\right) \longrightarrow 0$ e $d\left(C_{n}, A\right) \longrightarrow 0$, existem $\lim _{n} g\left(x^{*} ; B_{n}\right)=L \in \mathbb{R}$ e $\lim _{n} g\left(x^{*} ; C_{n}\right)=M \in \mathbb{R}$.Entào

$$
0 \leq|L-M|=\left|\lim _{n \longrightarrow \infty} g\left(x^{*} ; B_{n}\right)-\lim _{n \longrightarrow \infty} g\left(x^{*} ; C_{n}\right)\right|=\lim _{n \in \mathbb{N}}\left|g\left(x^{*} ; B_{n}\right)-g\left(x^{*} ; C_{n}\right)\right| .
$$


Sabendo que $d\left(B_{n}, C_{n}\right) \leq d\left(B_{n}, A\right)+d\left(A, C_{n}\right)$ temos $d\left(B_{n}, C_{n}\right) \longrightarrow 0$ e pelo lema 3.2 , $\left|\left(g\left(x^{*} ; B_{n}\right)-g\left(x^{*} ; C_{n}\right)\right)\right| \longrightarrow 0$. Portanto $L=M$, e com isto o limite independe da seqüência $\left\{B_{n}\right\}$.

Podemos definir $G\left(x^{*} ; A\right)=\lim _{n \longrightarrow \infty} g\left(x^{*} ; B_{n}\right)$, para quaisquer $x^{*} \in X^{*}$ e $A \in \Sigma_{1}$.

Fixamos $x^{*} \in X^{*}$.

(ii) Seja $B \in \Sigma$. Podemos tomar $B_{n}=B$ para todo $n \in \mathbb{N}$.

Logo $G\left(x^{*} ; B\right)=\lim _{n \rightarrow \infty} g\left(x^{*} ; B_{n}\right)=g\left(x^{*} ; B\right)$.

(iii) Queremos provar que $\lim _{\mu(E) \rightarrow 0}\left|G\left(x^{*} ; E\right)\right|=0$.

Dado $\varepsilon>0$, pela demonstração do Lema 3.2 , existe $\delta_{\varepsilon}>0$ tal que se $E \in \Sigma$ e $\mu(E) \leq \delta_{\varepsilon}$ então $\left|g\left(x^{*} ; E\right)\right|<\varepsilon\left\|x^{*}\right\|$.

Seja $A \in \Sigma_{1} \operatorname{com} \mu(A)<\delta=\frac{\delta_{\varepsilon}}{2}$.

Como $\Sigma$ é denso em $\Sigma_{1}$ por 1.38, para cada $n \in \mathbb{N}$, existe $B_{n} \in \Sigma$ tal que $d\left(A, B_{n}\right)<\frac{\delta}{n}$. Temos então $B_{n} \stackrel{n}{\longrightarrow} A$. Mas $B_{n} \subset A \cup\left(A \triangle B_{n}\right), \log 0 \mu\left(B_{n}\right)<\delta+\frac{\delta}{n} \leq \delta_{\varepsilon}$.

Portanto $\left|g\left(x^{*} ; B_{n}\right)\right|<\varepsilon\left\|x^{*}\right\|$ para qualquer $n \in \mathbb{N}$. Assim, $\mu(A)<\delta$ implica que $\left|G\left(x^{*} ; A\right)\right|=\lim _{n \rightarrow \infty}\left|g\left(x^{*} ; B_{n}\right)\right| \leq \varepsilon\left\|x^{*}\right\|$, ou seja, $\lim _{\mu(E) \rightarrow 0}\left|G\left(x^{*} ; E\right)\right|=0$.

(iv) Provaremos que $G\left(x^{*}, \cdot\right)$ é uniformemente contínua em $\Sigma_{1}$ com respeito a $d$.

Dado $\varepsilon>0$, existe $\delta_{1}>0$ tal que se $d(C, D)<\delta_{1} \operatorname{com} C, D$ em $\Sigma$ então

$\left|g\left(x^{*} ; C\right)-g\left(x^{*} ; D\right)\right|<\varepsilon\left\|x^{*}\right\|$, pela demonstração do lema 3.2

Sejam $\delta_{0}=\frac{\delta_{1}}{3}$ e $A, B$ em $\Sigma_{1}$ tais que $d(A, B)<\delta_{0}$.

Existem

$\delta_{A}>0$ tal que se $d\left(A, C^{\prime}\right)<\delta_{A}$ e $C^{\prime} \in \Sigma$ então $\left|G^{\prime}\left(x^{*} ; A\right)-g\left(x^{*} ; C\right)\right|<\varepsilon$ e

$\delta_{B}>0$ tal que se $d(B, D)<\delta_{B}$ e $D \in \Sigma$ então $\left|G\left(x^{*} ; B\right)-g\left(x^{*} ; D\right)\right|<\varepsilon$.

Pela densidade, existem $C, D$ em $\Sigma$ tais que

$$
\begin{gathered}
d(A, C)<\delta_{A} \text { e } d(A, C)<\delta_{0} \\
\text { e } d(B, D)<\delta_{B} \text { e } d(B, D)<\delta_{0} .
\end{gathered}
$$

Então $d(C, D) \leq d(C, A)+d(A, B)+D(B, D)<3 \delta_{0}<\delta_{1}$, e portanto

$\left|g\left(x^{*} ; C^{\prime}\right)-g\left(x^{*} ; D\right)\right|<\varepsilon\left\|x^{*}\right\|$.

Como 


$$
\left|G\left(x^{*} ; A\right)-G\left(x^{*} ; B\right)\right| \leq\left|G\left(x^{*} ; A\right)-g\left(x^{*} ; C^{\prime}\right)\right|+\left|g\left(x^{*} ; C^{\prime}\right)-g\left(x^{*} ; D\right)\right|+\left|g\left(x^{*} ; D\right)-G\left(x^{*} ; B\right)\right|,
$$

obtemos finalmente que se $A, B \in \Sigma_{1} \operatorname{com} d(A, B)<\delta_{0}$ então

$$
\left|G\left(x^{*} ; A\right)-G\left(x^{*} ; B\right)\right|<2 \varepsilon+\varepsilon .\left\|x^{*}\right\| .
$$

(v) Vamos mostrar que $G\left(x^{*} ; \cdot\right)$ é medida real $\sigma$-aditiva.

Para isto vamos provar que é finitamente aditiva e depois usamos sua $\mu$-continuidade dada por (iii).

Inicialmente temos $G\left(x^{*}, \emptyset\right)=0$, pois $\emptyset \in \Sigma$ e $g\left(x^{*}, \emptyset\right)=0$.

Dados $A$ e $B$ em $\Sigma_{1}$ disjuntos, consideremos $\left\{C_{n}\right\}$ e $\left\{D_{n}\right\}$ em $\Sigma$ tais que $C_{n} \stackrel{d}{\longrightarrow} A$ e $D_{n} \stackrel{d}{\longrightarrow} B$, ou seja, $\mu\left(A \triangle C_{n}\right) \longrightarrow 0$ e $\mu\left(B \triangle D_{n}\right) \longrightarrow 0$. Então

$$
G\left(x^{*} ; A\right)+G\left(x^{*} ; B\right)=\lim _{n \longrightarrow \infty}\left[g\left(x^{*} ; C_{n}\right)+g\left(x^{*} ; D_{n}\right)\right]
$$

Mas $g\left(x^{*} ; C_{n}\right)+g\left(x^{*} ; D_{n}\right)=g\left(x^{*} ; C_{n} \cup D_{n}\right)-g\left(x^{*} ; C_{n} \cap D_{n}\right)$, para todo $n \in \mathbb{N}$, já que $g\left(x^{*} ; \cdot\right)$ é medida real.

Pelo lema 1.36 temos $C_{n} \cup D_{n} \stackrel{d}{\longrightarrow} A \cup B$ e $C_{n}^{\prime} \cap D_{n} \stackrel{d}{\longrightarrow} A \cap B=\emptyset$.

Daí $G\left(x^{*} ; A\right)+G\left(x^{*} ; B\right)=G\left(x^{*} ; A \cup B\right)$ e $G\left(x^{*} ; \cdot\right)$ é finitamente aditiva.

Seja dada uma seqüência disjunta $\left\{A_{n}\right\}$ em $\Sigma_{1}$. Como $G\left(x^{*} ; \cdot\right)$ é finitamente aditiva $\left|G\left(x^{*} ; \bigcup_{n=1}^{\infty} A_{n}\right)-\sum_{n=1}^{r} G\left(x^{*} ; A_{n}\right)\right|=\left|G\left(x^{*} ; \bigcup_{n=r+1}^{\infty} A_{n}\right)\right|$.

Mas $\mu\left(\bigcup_{n=r+1}^{\infty} A_{n}\right) \stackrel{r \longrightarrow \infty}{\longrightarrow} 0$ e então por (iii) $\left|G\left(x^{*} ; \bigcup_{n=r+1}^{\infty} A_{n}\right)\right|^{r \longrightarrow \infty} \longrightarrow 0$.

Logo $G\left(x^{*} ; \cdot\right)$ é $\sigma$-aditiva.

Lema 3.4 Sejam $\Sigma, M_{0}, g, \mu$ e $G$ como no lema 3.3. Então para cada $A \in \Sigma_{1}$ a função $G(\cdot ; A): X^{*} \longrightarrow \mathbb{R}$ é convexa, positivamente homogênea e $\omega^{*}$-semicontínua inferiormente.

\section{prova:}

Fixado $A \in \Sigma_{1}$, fixemos também $\left\{B_{n}\right\}$ em $\Sigma$ com $d\left(B_{n}, A\right) \longrightarrow 0$.

Para todo $x^{*} \in X^{*}$ temos $G\left(x^{*} ; A\right)=\lim _{n \longrightarrow \infty} g\left(x^{*} ; B_{n}\right)$.

Sabemos que $G\left(x^{*} ; A\right) \in \mathbb{R}$ para todo $x^{*} \in X^{*}$ e então $G(\cdot ; A)$ é função real.

Como $g\left(x^{*} ; B_{n}\right)$ é uma função convexa para cada $n$, dados $x^{*}, y^{*} \in X^{*}$ e $0<\lambda<1$ temos que $\lim _{n \longrightarrow \infty} g\left(\left(\lambda x^{*}+(1-\lambda) y^{*}\right) ; B_{n}\right) \leq \lambda \lim _{n \rightarrow \infty} g\left(x^{*} ; B_{n}\right)+(1-\lambda) \lim _{n \longrightarrow \infty} g\left(y^{*} ; B_{n}\right)$

Logo, $G\left(\lambda x^{*}+(1-\lambda) y^{*} ; A\right) \leq \lambda G\left(x^{*} ; A\right)+(1-\lambda) G\left(y^{*} ; A\right)$ e $G(\cdot, A)$ é convexa.

Sendo $G\left(\lambda x^{*} ; A\right)=\lim _{n \longrightarrow \infty} g\left(\lambda x^{*} ; B_{n}\right)=\lim _{n \rightarrow \infty} \sup _{x \in M_{0}\left(B_{n}\right)}\left(\lambda x^{*}\right)(x)$, para todo $\lambda>0$ temos $\lim _{n \longrightarrow \infty} \lambda \sup _{x \in M_{0}\left(B_{n}\right)} x^{*}(x)=\lambda \lim _{n \longrightarrow \infty} g\left(x^{*} ; B_{n}\right)=\lambda G\left(x^{*} ; A\right)$ e $G(\cdot ; A)$ é positivamente homogênea. 
Para mostrar que $G(\cdot ; A)$ é $\omega^{*}$-semicontínua inferiormente, provaremos que $[G(\cdot ; A) \leq \lambda]$ é $\omega^{*}$-fechado, para todo $\lambda \in \mathbb{R}$.

Fixado $\lambda \in \mathbb{R}$, como $[G(\cdot ; A) \leq \lambda]$ é convexo, pelo teorema 1.8 basta mostrar que para todo $r \geq 0,[G(\cdot ; A) \leq \lambda] \cap r B_{X^{*}}$ é $\omega^{*}$-fechado, onde $r B_{X^{*}}=\left\{x^{*} \in X^{*} /\left\|x^{*}\right\| \leq r\right\}$.

Quando $r=0$ ficamos com um dos dois conjuntos $[G(\cdot ; A) \leq \lambda] \cap\{0\}=\emptyset$ ou $[G(\cdot ; A) \leq \lambda] \cap\{0\}=\{0\}$, e ambos são $\omega^{*}$-fechados.

Fixemos então $r>0$ e seja dado um net $\left\{x_{\alpha}^{*}\right\} \operatorname{com} x_{\alpha}^{*} \in[G(\cdot ; A) \leq \lambda] \cap r B_{X^{*}}$, para todo $\alpha$, e $x_{\alpha}^{*} \stackrel{w^{*}}{\longrightarrow} x_{0}^{*}$. Então $\left\|x_{0}^{*}\right\| \leq r$ e queremos mostrar que $x_{0}^{*} \in[G(\cdot ; A) \leq \lambda]$.

Dado $\varepsilon>0$, pela demonstração do lema 3.2 existe $\delta_{\varepsilon}>0$ tal que, para $B, C \in \Sigma$ com $d(B, C)<\delta_{\varepsilon},\left|g\left(x^{*} ; B\right)-g\left(x^{*} ; C\right)\right| \leq \frac{\varepsilon\left\|x^{*}\right\|}{3 r}$, para qualquer $x^{*} \in X^{*}$.

Lembrando que $d\left(B_{n}, A\right) \longrightarrow 0$, existe $n_{0} \in \mathbb{N}$ tal que para $m, n \geq n_{0} d\left(B_{n}, B_{m}\right)<\delta_{\varepsilon}$. Então para, $m, n \geq n_{0}, \sup _{x^{*} \in r B_{X^{*}}}\left|g\left(x^{*} ; B_{n}\right)-g\left(x^{*} ; B_{m}\right)\right|<\frac{\varepsilon}{3}$.

Fazendo $n=n_{0}$ e $m \longrightarrow \infty$, temos

$$
\sup _{x^{*} \in r B_{X^{*}}}\left|g\left(x^{*} ; B_{n_{0}}\right)-G\left(x^{*} ; A\right)\right| \leq \frac{\varepsilon}{3} .
$$

Como $g\left(\cdot ; B_{n_{0}}\right)$ é $\omega^{*}$-semicontínua inferiormente em $x_{0}^{*}$, existe uma vizinhança $\omega^{*}$ de $x_{0}^{*}$, que denotaremos por $V_{x_{0}^{*}}$, tal que se $x^{*} \in V_{x_{0}^{*}}$ então

$$
g\left(x^{*} ; B_{n_{0}}\right)-g\left(x_{0}^{*} ; B_{n_{0}}\right) \geq-\frac{\varepsilon}{3} .
$$

De (I) e (II) e

$G\left(x^{*} ; A\right)-G\left(x_{0}^{*} ; A\right)=G\left(x^{*} ; A\right)-g\left(x^{*} ; B_{n_{0}}\right)+g\left(x^{*} ; B_{n_{0}}\right)-g\left(x_{0}^{*} ; B_{n_{0}}\right)+g\left(x_{0}^{*} ; B_{n_{0}}\right)-G\left(x_{0}^{*} ; A\right)$, temos que se $x^{*} \in V_{x_{0}^{*}} \cap r B_{X^{*}}, G\left(x^{*} ; A\right)-G\left(x_{0}^{*} ; A\right) \geq-\frac{\varepsilon}{3}-\frac{\varepsilon}{3}-\frac{\varepsilon}{3}=-\varepsilon$, ou seja, $G\left(x_{0}^{*} ; A\right)<G\left(x^{*} ; A\right)+\varepsilon$.

Por outro lado, dado $V_{x_{0}^{*}}$ existe $\alpha_{0}$ tal que para $\alpha \geq \alpha_{0}$ temos $x_{\alpha}^{*} \in V_{x_{0}^{*}}$ pois $x_{\alpha}^{*} \stackrel{w^{*}}{\longrightarrow} x_{0}^{*}$.

Como $x_{\alpha}^{*} \in r B_{X^{*}}$ para todo $\alpha$, então $\alpha \geq \alpha_{0}$ implica que $x_{\alpha}^{*} \in V_{x_{0}^{*}} \cap r B_{X^{*}}$ e daí, em particular, $G\left(x_{0}^{*} ; A\right)<G\left(x_{\alpha_{0}}^{*} ; A\right)+\varepsilon$. Mas $G\left(x_{\alpha_{0}}^{*} ; A\right) \leq \lambda$ e então $G\left(x_{0}^{*} ; A\right) \leq \lambda+\varepsilon$

Sendo $\varepsilon$ arbitrário temos $G\left(x_{0}^{*} ; A\right) \leq \lambda$.(III)

Portanto $[G(\cdot ; A) \leq \lambda] \cap r B_{X^{*}}$ é $\omega^{*}$-fechado para todo $\lambda \in \mathbb{R}$ e todo $r \geq 0$ e pelo teorema 1.8 temos que $[G(\cdot ; A) \leq \lambda]$ é $\omega^{*}$-fechado.

Teorema 3.5 Sejam $\Sigma$ uma álgebra de subconjuntos de $\Omega, \Sigma_{1}$ a $\sigma$-álgebra gerada por $\Sigma$ e $M_{0}: \Sigma \longrightarrow P_{l f c}(\mathrm{X})$ uma multimedida que também seja multimedida fraca. Se existe uma medida real não negativa $\sigma$-aditiva $\mu \mathrm{em} \Sigma_{1}$ tal que $\left.M_{0} \ll \mu\right|_{\Sigma}$ então $M_{0}$ pode ser estendida para uma multimedida fraca $M: \Sigma_{1} \longrightarrow P_{l f c}(X)$. 
prova:

Podemos utilizar o lema 3.3 e definir $M: \Sigma_{1} \longrightarrow \mathcal{P}(X)$ por

$$
M(A)=\left\{x \in X / x^{*}(x) \leq G\left(x^{*} ; A\right), \forall x^{*} \in X^{*}\right\}, A \in \Sigma_{1}
$$

Fixado $A \in \Sigma_{1}$, vamos mostrar que $M(A) \in P_{l f c}(X)$.

Sendo $g: X^{*} \longrightarrow \mathbb{R}$ dada por $g\left(x^{*}\right)=G\left(x^{*}, A\right)$, podemos aplicar a proposição 1.33 , pois $g$ é convexa, própria, positivamente homogênea e $\omega^{*}$-semicontínua inferiormente e obtemos $K \subset X$ não vazio, convexo e $\omega$-fechado, e portanto fechado, tal que $\left.g^{*}\right|_{\widehat{X}}$ é indicadora de $K$. Então

$$
\begin{aligned}
& K=\quad\left\{x \in X: g^{*}(\widehat{x}) \leq 0\right\} \quad=\left\{x \in X: \sup _{x^{*} \in X^{*}}\left(x^{*}(x)-G\left(x^{*}, A\right)\right) \leq 0\right\} \\
& =\left\{x \in X: x^{*}(x) \leq G\left(x^{*}, A\right), \forall x^{*} \in X^{*}\right\}=x^{*} \in X^{*} M(A),
\end{aligned}
$$

donde $M(A) \in P_{f c}(X)$.

Resta mostrar que $M(A)$ é limitado. Consideremos $\widehat{M(A)} \subset X^{* *}$. Para todo $x^{*} \in X^{*} \mathrm{e}$ todo $x \in M(A)$ temos $x^{*}(x) \leq G\left(x^{*}, A\right)$ e $-x^{*}(x) \leq G\left(-x^{*}, A\right)$, de modo que $\left|\widehat{x}\left(x^{*}\right)\right| \leq M_{x^{*}}=$ $\max \left\{G\left(x^{*}, A\right), G\left(-x^{*}, A\right)\right\} \in \mathbb{R}$. Pelo princípio da limitação uniforme ([3],p66), existe $L \in \mathbb{R}$ com $\|\widehat{x}\| \leq L$, para todo $\widehat{x} \in \widehat{M(A)}$. Como $\|x\|=\|\widehat{x}\|$, segue-se que $M(A)$ é limitado.

Além disso, devemos verificar que $M$ coincide com $M_{0}$ em $\Sigma$.

Para A $\in \Sigma$ temos $G\left(x^{*} ; A\right)=g\left(x^{*} ; A\right)=\sigma\left(x^{*} ; M_{0}(A)\right)$, e como para $x \in M_{0}(A)$, $x^{*}(x) \leq g\left(x^{*} ; A\right)$ para todo $x^{*} \in X^{*}$, temos que $M_{0}(A) \subset \mathrm{M}(\mathrm{A})$.

Suponhamos que não vale $M(A) \subset M_{0}(A)$. Então existe $x_{0} \in M(A)$ com $x_{0} \notin M_{0}(A)$. Temos que $\left\{x_{0}\right\}$ é um conjunto convexo e compacto e $M_{0}(A)$ é fechado e convexo.

Pelo teorema 1.6 , existem $c, a$ constantes reais e $x^{*} \in X^{*}$ tais que

$$
x^{*}(y) \leq c<a<x^{*}\left(x_{0}\right) \text {, para todo } y \in M_{0}(A)
$$

e $\operatorname{assim} \sup _{y \in M_{0}(A)} x^{*}(y) \leq c<a<x^{*}\left(x_{0}\right)$. Com isto temos $g\left(x^{*} ; A\right)<x^{*}\left(x_{0}\right)$, o que é absurdo, pois $g\left(x^{*} ; A\right)=G\left(x^{*} ; A\right) \geq x^{*}\left(x_{0}\right)$. Logo $M(A) \subset M_{0}(A)$.

Portanto, $M(A)=M_{0}(A)$, para todo $A \in \Sigma$.

Falta provarmos que $M$ é uma multimedida fraca, isto é, para todo $x^{*} \in X^{*}, \sigma\left(x^{*}, M(\cdot)\right)=$ $\sup _{x} x^{*}(x): \Sigma_{1} \longrightarrow \mathbb{R} \cup\{\infty\}$ é medida $\sigma$-aditiva com sinal. $x \in M(\cdot)$

Em primeiro lugar, sabemos que $\sigma\left(x^{*}, M(\cdot)\right)$ tem valores reais, pois $\sup _{x \in M(A)} x^{*}(x) \leq G\left(x^{*}, A\right)$ e $G\left(x^{*}, A\right) \in \mathbb{R}$, para quaisquer $x^{*} \in X^{*}$ e $A \in \Sigma_{1}$. 
Vamos provar que $\sigma\left(x^{*}, M(\cdot)\right)=G\left(x^{*}, \cdot\right)$ para todo $x^{*} \in X^{*}$ e daí, pelo lema 3.3(iv), seguirá o que queremos.

Inicialmente, vamos mostrar que para $A, B \in \Sigma_{1}$ disjuntos vale

$$
M(A \cup B)=\overline{M(A)+M(B)} .
$$

De fato, dado $x \in M(A)+M(B)$ temos $x=x_{A}+x_{B}$, com $x_{A} \in M(A)$ e $x_{B} \in M(B)$. Então, para todo $x^{*} \in X^{*}$ temos $x^{*}(x)=x^{*}\left(x_{A}\right)+x^{*}\left(x_{B}\right) \leq G\left(x^{*}, A\right)+G\left(x^{*}, B\right)=G\left(x^{*}, A \cup B\right)$, pois $G\left(x^{*}, \cdot\right)$ é medida. Logo, $x \in M(A \cup B)$ e temos $\overline{M(A)+M(B)} \subset M(A \cup B)$, pois $M(A \cup B)$ é fechado.

Por outro lado, se $x \in M(A \cup B)$ e $x \notin \overline{M(A)+M(B)}$ pelo teorema 1.6, existem $x_{0}^{*} \in X^{*}$ e $c \in \mathbb{R}$ com $x_{0}^{*}(x)>c>x_{0}^{*}(y)$, para todo $y \in \overline{M(A)+M(B)}$. Em particular,para todo $y=x_{A}+x_{B}$ com $x_{A} \in M(A)$ e $x_{B} \in M(B)$ vale $x_{0}^{*}(x)>c>x_{0}^{*}\left(x_{A}\right)+x_{0}^{*}\left(x_{B}\right)$. Daí $x_{0}^{*}(x)>$ $c \geq G\left(x_{0}^{*}, A\right)+G\left(x_{0}^{*}, B\right)=G\left(x_{0}^{*}, A \cup B\right)$, o que contradiz $x \in M(A \cup B)$. Então $M(A \cup B) \subset$ $\overline{M(A)+M(B)}$.

Fixemos agora $x^{*} \in X^{*}$ e vamos verificar que $\sigma\left(x^{*}, M(\cdot)\right)$ é aditiva. Dados $A, B \in \Sigma_{1}$ disjuntos, temos

$$
\begin{aligned}
& \sigma\left(x^{*}, M(A \cup B)\right)=\sup _{x \in M(A \cup B)} x^{*}(x)=\sup _{x \in \frac{M(A)+M(B)}{M(A)}} x^{*}(x) \\
& =\sup _{x \in M(A)+M(B)} x^{*}(x)=\sup _{x_{A} \in M(A), x_{B} \in M(B)}\left(x^{*}\left(x_{A}\right)+x^{*}\left(x_{B}\right)\right) \\
& =\sup _{x \in M(A)} x^{*}(x)+\sup _{x \in M(B)} x^{*}(x)=\sigma\left(x^{*}, M(A)\right)+\sigma\left(x^{*}, M(B)\right) \text {. }
\end{aligned}
$$

Vamos finalmente provar que $\sigma\left(x^{*}, M(A)\right)=G\left(x^{*}, A\right)$, para todo $A \in \Sigma_{\mathbf{1}}$.

Fixamos $A \in \Sigma_{1}$ e uma seqüência $\left\{B_{n}\right\}$ em $\Sigma \operatorname{com} d\left(B_{n}, A\right) \stackrel{n}{\longrightarrow} 0$, ou $\mu\left(A \triangle B_{n}\right) \stackrel{n}{\longrightarrow} 0$.

Como $\sigma\left(x^{*}, M(\cdot)\right)$ é aditiva e real, para todo $n \in \mathbb{N}$ temos

$$
\begin{aligned}
\sigma\left(x^{*}, M(A)\right) & =\sup _{x \in M(A)} x^{*}(x) \\
& =\sup _{x \in M\left(A \cap B_{n}\right)} x^{*}(x)+\sup _{x \in M\left(A \cap B_{n}^{c}\right)} x^{*}(x) \\
& =\sup _{x \in M\left(A \cap B_{n}\right)} x^{*}(x)+\sup _{x \in M\left(A^{c} \cap B_{n}\right)} x^{*}(x)+\sup _{x \in M\left(A \cap B_{n}^{c}\right)} x^{*}(x)-\sup _{x \in M\left(A^{c} \cap B_{n}\right)} x^{*}(x) \\
& =\sup _{x \in M\left(B_{n}\right)} x^{*}(x)+\sup _{x \in M\left(A \cap B_{n}^{c}\right)} x^{*}(x)+\inf _{x \in M\left(A^{c} \cap B_{n}\right)}-x^{*}(x) \\
& =\sigma\left(x^{*}, M\left(B_{n}\right)\right)+\sup _{x \in M\left(A \cap B_{n}^{c}\right)} x^{*}(x)+\inf _{x \in M\left(A^{c} \cap B_{n}\right)}-x^{*}(x)
\end{aligned}
$$

e, de forma análoga,

$$
\sigma\left(x^{*}, M\left(B_{n}\right)\right)=\sigma\left(x^{*}, M(A)\right)+\sup _{x \in M\left(A^{c} \cap B_{n}\right)} x^{*}(x)+\inf _{x \in M\left(A \cap B_{n}^{c}\right)}-x^{*}(x) .
$$

Da definiçào de $M$ obtemos, para qualquer $C \in \Sigma_{1}$ e qualquer $y^{*} \in X^{*}$, que

$$
\sup _{x \in M(C)} y^{*}(x) \leq G\left(y^{*}, C\right) \text { e } \inf _{x \in M(C)} y^{*}(x) \leq G\left(y^{*}, C^{\prime}\right) \text {. }
$$


Utilizando essas desigualdades com $A \cap B_{n}^{c}, A^{c} \cap B_{n}, x^{*}$ e $-x^{*}$ ficamos com $\left|\sigma\left(x^{*}, M(A)\right)-\sigma\left(x^{*}, M\left(B_{n}\right)\right)\right| \leq \max \left\{\left|G\left(x^{*}, A \cap B_{n}^{c}\right)\right|+\left|G\left(-x^{*}, A^{c} \cap B_{n}\right)\right|,\left|G\left(x^{*}, A^{c} \cap B_{n}\right)\right|+\right.$ $\left.\left|G\left(-x^{*}, A \cap B_{n}^{c}\right)\right|\right\}$.

Lembrando que $\sigma\left(x^{*}, M\left(B_{n}\right)\right)=g\left(x^{*}, B_{n}\right)$, para todo $n \in \mathbb{N}$, que $\lim _{\mu(E) \rightarrow 0}\left|G\left(x^{*}, E\right)\right|=0$ e $\lim _{\mu(E) \longrightarrow 0}\left|G\left(-x^{*}, E\right)\right|=0$ (por 3.3(iii)) e que $\mu\left(A \cap B_{n}^{c}\right), \mu\left(A^{c} \cap B_{n}\right) \stackrel{n}{\longrightarrow} 0$, pois $A \cap B_{n}^{c}$ e $A^{c} \cap B_{n}$ estão contidos em $A \triangle B_{n}$ e $\mu\left(A \triangle B_{n}\right) \stackrel{n}{\longrightarrow} 0$, obtemos que $\sigma\left(x^{*}, M(A)\right)=\lim _{n \rightarrow \infty} g\left(x^{*}, B_{n}\right)$.

Mas esse limite é exatamente $G\left(x^{*}, A\right)$ e $\operatorname{assim} G\left(x^{*}, A\right)=\sigma\left(x^{*}, M(A)\right)$.

Portanto, $M$ é uma multimedida fraca.

Teorema 3.6 Sejam $\Sigma$ uma álgebra de subconjuntos de $\Omega$ e $\Sigma_{1}$ a $\sigma$-álgebra gerada por $\Sigma$. Se $X$ for reflexivo e $M_{0}: \Sigma \longrightarrow P_{w k c}(\mathrm{X})$ for uma multimedida fraca, então as seguintes afirmações são equivalentes:

(i) $M_{0}$ pode se estendida para uma multimedida forte $M: \Sigma_{1} \longrightarrow P_{w k c}(X)$.

(ii) $M_{0}(\Sigma)$ é um subconjunto relativamente fraco-compacto.

(iii) $M_{0}$ é fortemente aditiva.

(iv) Existe uma medida $\mu: \Sigma_{1} \longrightarrow \mathbb{R}$ não negativa $\sigma$-aditiva tal que $\left.M_{0} \ll \mu\right|_{\Sigma}$.

\section{prova:}

Inicialmente, por $M_{0}$ ter valores em $P_{w k c}(X)$, pela proposição 2.13 segue que $M_{0}$ é multimedida, pois $\overline{M_{0}(A)+M_{0}(B)}=M_{0}(A)+M_{0}(B)$.

(i) $\Rightarrow$ (ii) Temos $M_{0}(\Sigma)=M(\Sigma) \subset M\left(\Sigma_{1}\right)$ e, pela proposição $2.21, M\left(\Sigma_{1}\right)$ é relativamente $\omega$-compacto. Então $M_{0}(\Sigma)$ é relativamente $\omega$-compacto.

(ii) $\Rightarrow$ (iii) Sendo $M_{0}(\Sigma)$ relativamente $\omega$-compacto, pelas proposições $2.13(1)$ e 2.17 temos que $M$ é fortemente aditiva, pois $P_{w k c}(X) \subset P_{f c}(X)$.

(iii) $\Rightarrow$ (iv) É a Proposição 2.20

(iv) $\Rightarrow$ (i) Pelo Teorema 3.5, $M_{0}$ pode ser estendida para uma multimedida fraca $M: \Sigma_{1} \longrightarrow P_{w k c}(X)$, pois $P_{w k c}(X)=P_{l f c}(X)$ porque $X$ é reflexivo.

Pela proposição $2.21, M$ é $\sigma$-aditiva, ou seja, é multimedida forte. 


\section{Capítulo 4}

\section{Integração com respeito a uma multimedida}

Este capítulo visa estabelecer condições para que possamos integrar uma multimedida, o fato desta integral poder ser usada para definir uma outra multimedida e descobrir quais as características da inicial que esta nova multimedida tem.

Neste capítulo $\Sigma$ será uma $\sigma$-álgebra de subconjuntos de $\Omega$.

Lembramos que $B(\Sigma)$ denota o conjunto das funções $f: \Omega \longrightarrow \mathbb{R}$ limitadas e mensuráveis e que se $f \in B(\Sigma)$ e $m \in b v(\Sigma, X)$ existe a integral $\int_{A} f d m$ para todo $A \in \Sigma$ pelo exemplo 2.7 .

Assim, se $M: \Sigma \longrightarrow P_{w k c}(X)$ for uma multimedida de variação limitada, temos pela proposição $2.30, S_{M} \subset b v(\Sigma, X)$ e portanto, para todo $m \in S_{M}$ e todo $A \in \Sigma$, existe $\int_{A} f d m$, o que nos permite enunciar a seguinte definição.

Definição 4.1 Sejam $M: \Sigma \longrightarrow P_{w k c}(X)$ multimedida de variação limitada e $f \in B(\Sigma)$. A integral de $f$ com respeito a $M$ em $A \in \Sigma$ é dada por $\int_{A} f d M=\left\{\int_{A} f d m: m \in S_{M}\right\}$.

Teorema 4.2 Sejam $f \in \mathrm{B}(\Sigma)$ e $\mathrm{M}: \Sigma \rightarrow P_{\text {wkc }}(X)$ uma multimedida forte de variação limitada. Então a multifunção de conjuntos definida por $G(A)=\int_{A} f d M$, para cada $A \in \Sigma$, é uma multimedida forte com valores em $P_{w k c}(X)$ e de variação limitada.

prova:

Primeiro mostraremos que $G(A) \in P_{w k c}(\mathrm{X})$ para cada $A \in \Sigma$.

Em primeiro lugar, $G(A) \neq \emptyset$, pois $S_{M} \neq \emptyset$, por 2.29 .

Seja $\left\{\int_{A} f d m_{\alpha}\right\}{ }_{\alpha \in D}$ um net em $G(A)$, onde $\left\{m_{\alpha}\right\}_{\alpha \in D}$ é um net em $S_{M}$. 
Desde que $S_{M}$ é $\tau$-compacto pelo Lema 2.33 , existe um subnet $\left\{m_{\alpha_{k}}\right\}_{k \in J}$ de $\left\{m_{\alpha}\right\}_{\alpha \in D}$ que $\tau$-converge para $m \in S_{M}$, ou seja, para todo $B \in \Sigma$ a net $\left\{m_{\alpha_{k}}(B)\right\}_{k \in J}$ é $\omega$-convergente para $m(B)$.

Observando que $\left|m_{\alpha_{k}}\right|(\Omega) \leq|M|(\Omega)<\infty$, temos por 2.9 que,

$\lim _{k \in J} x^{*}\left(\int_{B} f d m_{\alpha_{k}}\right)=\lim _{k \in J} \int_{B} f d x^{*} \circ m_{\alpha_{k}}=\int_{B} f d x^{*} \circ m=x^{*}\left(\int_{B} f d m\right)$.

Logo $G(A)$ é fraco-compacto.

Agora vamos mostrar que $G(A)$ é convexo.

Sejam $x, y \in G(A)$ onde $x=\int_{A} f d m_{1}$ e $y=\int_{A} f d m_{2}$, com $m_{1}, m_{2} \in S_{M}$. Temos $\lambda x+(1-\lambda) y=\lambda \int_{A} f d m_{1}+(1-\lambda) \int_{A} f d m_{2}=\int_{A} f\left[\lambda d m_{1}+(1-\lambda) d m_{2}\right]=\int_{A} f d\left[\lambda m_{1}+(1-\lambda) m_{2}\right]$.

Sendo $m=\lambda m_{1}+(1-\lambda) m_{2}$, temos que $m$ é medida vetorial. Além disso, como $m_{1}, m_{2}$ pertencem a $S_{M}$ temos que $m_{1}(A), m_{2}(A) \in M(A)$ e este sendo convexo vale que

$$
m(A)=\lambda m_{1}(A)+(1-\lambda) m_{2}(A) \in M(A) .
$$

Logo, $\int_{A} f d m \in G(A)$ e $G(A)$ é convexo.

Então $G(A) \in P_{w k c}$.

Vamos provar que $G$ é uma multimedida forte.

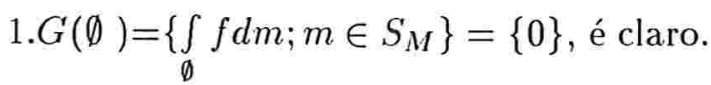

2. $G\left(\cup_{n \in \mathbb{N}} A_{n}\right)=\sum_{n \in \mathbb{N}} G\left(A_{n}\right)$, para $\left\{A_{n}\right\}_{n \in \mathbb{N}}$ seqüência disjunta em $\Sigma$.

Mostraremos inicialmente que $\sigma\left(x^{*}, G(\cdot)\right)$ é $\sigma$-aditiva para todo $x^{*} \in X^{*}$.

Sejam $x^{*} \in X^{*}$ e $A, B \in \Sigma$ disjuntos. Então temos que

$$
\begin{aligned}
\sigma\left(x^{*}, G(A \cup B)\right) & =\sigma\left(x^{*},\left\{\int_{A \cup B} f d m: m \in S_{M}\right\}\right) \\
& =\sup _{m \in S_{M}} x^{*}\left(\int_{A \cup B} f d m\right) \\
& =\sup _{m \in S_{M}} x^{*}\left(\int_{A} f d m+\int_{B} f m\right) .
\end{aligned}
$$

Logo,

$$
\begin{aligned}
\sigma\left(x^{*}, G(A \cup B)\right. & \leq \sup _{m \in S_{M}} x^{*}\left(\int_{A} f d m\right)+\sup _{m \in S_{M}} x^{*}\left(\int_{B} f d m\right) \\
& =\sigma\left(x^{*}, G(A)\right)+\sigma\left(x^{*}, G(B)\right) .
\end{aligned}
$$

Como $G(A), G(B)$ são $\omega$-compactos, existem $m_{1}, m_{2} \in S_{M}$ tais que 


$$
\sigma\left(x^{*}, G(A)\right)=x^{*}\left(\int_{A} f d m_{1}\right) \text { e } \sigma\left(x^{*}, G(B)\right)=x^{*}\left(\int_{B} f d m_{2}\right)
$$

Pela Observação 2.29 sabemos que $S_{M}$ é decomponível e assim a medida $m_{0}$ dada por $m_{0}=\chi_{A_{1}} m_{1}+\chi_{A_{2}} m_{2}$ é um seletor de $M$.

Como $\int_{A \cup B} f d m_{0}=\int_{A} f d m_{1}+\int_{B} f d m_{2}$, vale que

$x^{*}\left(\int_{A \cup B} f d m_{0}\right)=x^{*}\left(\int_{A} f d m_{1}+\int_{B} f d m_{2}\right)=x^{*}\left(\int_{A} f d m_{1}\right)+x^{*}\left(\int_{B} f d m_{2}\right) \stackrel{(\mathrm{II})}{=} \sigma\left(x^{*}, G(A)\right)+$ $\sigma\left(x^{*}, G(B)\right)$.

Logo,

$$
\sup _{m \in S_{M}} x^{*}\left(\int_{A \cup B} f d m\right) \geq \sigma\left(x^{*}, G(A)\right)+\sigma\left(x^{*}, G(B)\right) .
$$

Por (I) e (III) vale que

$$
\sigma\left(x^{*}, G(A \cup B)\right)=\sigma\left(x^{*}, G(A)\right)+\sigma\left(x^{*}, G(B)\right),
$$

ou seja, $\sigma\left(x^{*}, G(\cdot)\right)$ é aditiva e pela proposiçào $2.13(2), G$ é aditiva, pois, como $G$ tem valores em $P_{w k c}(X)$, para $A, B \in \Sigma$ disjuntos temos $\overline{G(A)+G(B)}=G(A)+G(B)$.

Para a $\sigma$-aditividade temos $\left|\sigma\left(x^{*}, G\left(\bigcup_{n=1}^{k} A_{n}\right)\right)-\sum_{n=1}^{\infty} \sigma\left(x^{*}, G\left(A_{n}\right)\right)\right|=$

$$
\left|\sigma\left(x^{*}, G\left(\bigcup_{n=1}^{\infty} A_{n}\right)\right)-\sigma\left(x^{*}, G\left(\bigcup_{n=1}^{k} A_{n}\right)\right)\right| .
$$

Como podemos escrever $\bigcup_{n=1}^{\infty} A_{n}=\left(\bigcup_{n=1}^{k} A_{n}\right) \cup\left(\bigcup_{n=k+1}^{\infty} A_{n}\right)$ e essa união é disjunta,

$$
\left|\sigma\left(x^{*}, G\left(\bigcup_{n=k+1}^{\infty} A_{n}\right)\right)\right|=\left|\sup _{m \in S_{M}} x^{*}\left(\int_{\substack{\bigcup \\ n=k+1}}^{\infty} A_{n} f d m\right)\right| .
$$

Mas $\left|\sup _{m \in S_{M}} x^{*}(\underbrace{\infty}_{\substack{\cup \\ n=k+1}} A_{n} f d m)\right| \leq \sup _{m \in S_{M}}\left|\int_{\substack{\bigcup_{n=k+1}^{\infty} \\ n=k n}} f d x^{*} \circ m\right| \leq \sup _{m \in S_{M}}\|f\|_{\infty}\left|x^{*} \circ m\right|\left(\bigcup_{n=k+1}^{\infty} A_{n}\right)$, e como $\left|x^{*} \circ m\right|(\cdot) \leq\left\|x^{*}|||m|(\cdot) \leq\right\| x^{*} \||M|(\cdot)$, por 2.4 e $2.30(\mathrm{i})$, então

$$
\left|\sigma\left(x^{*}, G\left(\bigcup_{n=1}^{\infty} A_{n}\right)\right)-\sigma\left(x^{*}, G\left(\bigcup_{n=1}^{k} A_{n}\right)\right)\right| \leq\|f\|_{\infty}\left\|x^{*}\right\| \cdot|M|\left(\bigcup_{n=k+1}^{\infty} A_{n}\right) \text {. Por 2.30(iii), }|M|(\cdot) \text { é }
$$
$\sigma$-aditiva e daí, fazendo $k \longrightarrow \infty$,

$$
\sigma\left(x^{*}, G\left(\bigcup_{n=1}^{\infty} A_{n}\right)\right)=\sum_{n=1}^{\infty} \sigma\left(x^{*}, G\left(A_{n}\right)\right),
$$

para todo $x^{*} \in X^{*}$.

Pela proposição $2.21, G$ é $\sigma$-aditiva, pois tem valores em $P_{w k c}(X)$. 
Além disso, temos que $G$ é de variaçào limitada, com $|G|(\Omega) \leq\|f\|_{\infty}|M|(\Omega)$. De fato,

$$
\begin{aligned}
|G|(\Omega) & =\sup _{\Pi} \sum_{A_{n} \in \Pi} h\left(\{0\}, G\left(A_{n}\right)\right)=\sup _{\Pi} \sum_{A_{n} \in \Pi} h\left(\{0\},\left\{\int_{A_{n}} f d m: m \in S_{M}\right\}\right) \\
& =\sup _{\Pi} \sum_{A_{n} \in \Pi} \sup _{m \in S_{M}}\left\|\int f d m\right\| \leq\|f\|_{\infty} \sup _{\Pi} \sum_{A_{n} \in \Pi} \sup _{m \in S_{M}}|m|\left(A_{n}\right) \\
& \leq\|f\|_{\infty} \sup _{\Pi} \sum_{A_{n} \in \Pi}|M|\left(A_{n}\right)=\|f\|_{\infty}|M|(\Omega),
\end{aligned}
$$

sendo que a penúltima desigualdade segue da proposição 2.30 .

Dada uma função $f \in B(\Omega)$ podemos escrever $f=f_{+}-f_{-}$onde $f_{+}=\max (f, 0)$ e $f_{-}=\max (-f, 0)$.

Teorema 4.3 Sejam $M: \Sigma \longrightarrow P_{w k c}(X)$ multimedida forte de variação limitada e $f$ em $B(\Omega)$. Então para cada $A$ em $\Omega$, cada $x^{*} \in X^{*}$ e $G$ dada no teorema 4.2 temos

$$
\sigma\left(x^{*}, G(A)\right)=\int_{A} f_{+} d \sigma\left(x^{*}, M(\cdot)\right)-\int_{A} f_{-} d \sigma\left(-x^{*}, M(\cdot)\right) .
$$

\section{prova:}

Provaremos primeiro para funções simples.

Seja $f: \Omega \longrightarrow \mathbb{R}$ simples. Então $f_{+}$e $f_{-}$também são funções simples e podemos escrever $f_{+}=\sum_{i=1}^{k} a_{i} \chi_{A_{i}}$ e $f_{-}=\sum_{j=1}^{\lambda}\left(-b_{j}\right) \chi_{B_{j}}$, com $a_{i},\left(-b_{j}\right)$ estritamente positivos e os conjuntos $A_{i}$ e $B_{j}$ disjuntos dois a dois, $1 \leq i \leq k, 1 \leq j \leq \lambda$.

Então $f=\sum_{i=1}^{k} a_{i} \chi_{A_{1}}+\sum_{j=1}^{\lambda} b_{j} \chi_{B}$, e, sendo $C=\bigcup_{i=1}^{k} A_{i}$ e $D=\bigcup_{j=1}^{\lambda} B_{j}, f(x)=0$ para $x \notin C^{\prime} \cup D$. Logo $\int_{A} f d M=\int_{A \cap(C \cup D)} f d M$, para cada $A \in \Sigma$.

Para $x^{*} \in X^{*}$ temos então

$$
\begin{aligned}
\sigma\left(x^{*}, G(A)\right) & =\sigma\left(x^{*}, G((A \cap C) \cup(A \cap D))\right) \\
& =\sigma\left(x^{*}, G(A \cap C)\right)+\sigma\left(x^{*}, G(A \cap D)\right) \\
& =\sigma\left(x^{*}, \int_{A \cap C} f d M\right)+\sigma\left(x^{*}, \int_{A \cap D} f d M\right),
\end{aligned}
$$

pois $\sigma\left(x^{*} ; G(\cdot)\right)$ é $\sigma$-aditiva.

Portanto,

$$
\sigma\left(x^{*}, G(A)\right)=\sup _{m \in S_{M}} x^{*}\left(\sum_{i=1}^{k} a_{i} m\left(A \cap A_{i}\right)\right)+\sup _{m \in S_{M}}\left(-x^{*}\right)\left(\sum_{j=1}^{\lambda}\left(-b_{j}\right) m\left(A \cap B_{j}\right)\right) \cdot(I)
$$


Como os $A_{i}{ }^{\prime} s$ e os $B_{j}{ }^{\prime} s$ são disjuntos,

$\sigma\left(x^{*}, G(A)\right)=\sum_{i=1}^{k} \sup _{m \in S_{M}} x^{*}\left(a_{i} m\left(A \cap A_{i}\right)\right)+\sum_{j=1}^{\lambda} \sup _{m \in S_{M}}\left(-x^{*}\right)\left(-b_{j} m\left(A \cap B_{j}\right)\right)$.

Mas $M(B)=\left\{m(B) / m \in S_{M}\right\}$, para todo $B \in \Sigma$, pelo teorema 2.36 , e então temos que

$$
\begin{aligned}
\sigma\left(x^{*}, G(A)\right) & =\sum_{i=1}^{k} a_{i} \sigma\left(x^{*}, M\left(A \cap A_{i}\right)\right)+\sum_{j=1}^{\lambda}\left(-b_{j}\right) \sigma\left(-x^{*}, M\left(A \cap B_{j}\right)\right) \\
& =\int_{A} \sum_{i=1}^{k} a_{i} \chi_{A_{i}} d \sigma\left(x^{*}, M(\cdot)\right)-\int_{A} \sum_{j=1}^{\lambda}\left(-b_{j}\right) \chi_{B_{j}} d \sigma\left(-x^{*}, M(\cdot)\right) .
\end{aligned}
$$

$\operatorname{Logo} \sigma\left(x^{*}, G(A)\right)=\int_{A} f_{+} d \sigma\left(x^{*}, M(\cdot)\right)-\int_{A} f_{-} d \sigma\left(-x^{*}, M(\cdot)\right)$.

Para o caso genérico de uma função $f$ mensurável e limitada, existem sequências de funções simples mensuráveis com $\left\{s_{n}^{+}\right\}$e $\left\{s_{n}^{-}\right\}$com $s_{n}^{+} \stackrel{u}{\longrightarrow} f_{+}$e $s_{n}^{-} \stackrel{u}{\longrightarrow} f_{-}$. Daí segue o resultado.

Agora que conseguimos definir uma multimedida pela integral de outra, gostariamos de saber qual a relação entre seus seletores. O próximo teorema trata disso.

Teorema 4.4 Sejam $M: \Sigma \longrightarrow P_{w k c}(X)$ multimedida forte de variação limitada, $f \in \mathrm{B}(\Omega)$ e $G: \Sigma \longrightarrow P_{w k c}(X)$, dada por $G(A)=\int_{A} f d M, A \in \Sigma$. Entào se $m_{0} \in S_{M}$, a medida vetorial $\sigma$-aditiva $n_{0}$ definida por $n_{0}(A)=\int_{A} f d m_{0}, A \in \Sigma$, é um seletor de $G$.

prova:

Para $A \in \Sigma, n_{0}(A)=\int_{A} f d m_{0} \in\left\{\int_{A} f d m: m \in S_{M}\right\}=\int_{A} f d M=G(A)$ e assim $n_{0}(A) \in G(A)$.

Dado $m_{0} \in S_{M}$, é claro que $n_{0}$ é medida vetorial pela proposição 2.10 e então $n_{0}$ é seletor de $G$. Além disso, como $m_{0}$ é $\sigma$-aditiva, $\left|m_{0}\right|(\cdot)$ também o é pela proposição 2.30 . Daí, se $\left\{A_{n}\right\}_{n \in \mathbb{N}}$ é seqüência disjunta em $\Sigma$,

$$
\begin{aligned}
\left\|n_{0}\left(\bigcup_{n \in \mathbb{N}} A_{n}\right)-\sum_{n=1}^{k} n_{0}\left(A_{n}\right)\right\| & =\left\|n_{0}\left(\bigcup_{n=k+1}^{\infty} A_{n}\right)\right\|=\left\|\int{\underset{\bigcup}{n=k+1}}_{n}^{\infty} A_{n} f d m_{0}\right\| \\
& \leq\|f\|_{\infty}\left|m_{0}\right|\left(\bigcup_{n=k+1}^{\infty} A_{n}\right) \stackrel{k \rightarrow \infty}{\longrightarrow} 0,
\end{aligned}
$$

e $n_{0}$ é $\sigma$-aditiva. 


\section{Referências Bibliográficas}

[1] BOURBAKI, N., Theory of sets, Elements of mathematics, Hermann, Paris, 1968.

[2] BREZIS, H., Analyse Fonctionelle, Théorie et applications, Masson, Paris, 1983.

[3] DLiNFORD, N. and SCHWARTZ, J. Linєar Operators I, Willey Interscience,New York, 1958.

[4] DIESTEL,J. and UHL,J., Vector Measures. Am. Math. Soc. Publ.,Providence, 1977.

[5] GODET-THOBIE, C. Multimesures et multimesures de transitions, Thèse, Montpellier, 1975.

[6] GODET-THOBIE, C. Some results about multimeasures and their selectors, Measure Theory at Oberwohlfach, 1979, Lectures Notes in Math., vol 794, Springer, Berlin and New York, 1979, pp 112-116.

[7] HEVITT, E., STROMBERG, K. Real and abstract analysis, Springer-Verlag, Berlin and New York, 1965.

[8] HOLMES, R.B., Geometric Functional Analysis and its applications, New York, Springer Verlag, 1975.

[9] KANDILAKIS, D., On the extension of multimeasures and integration with respect to a multimeasures, Proc. Am. Math. Soc, n.1,pp 85-93,1992.

[10] KELLEY,JOHN L., General Topology, Van Nostrand,New York, 1955.

[11] MURKHEJEA,A. and POTOVEn,K., Real and Functional Analysis, Part A:Real Analysis, Plenum Press, New York and Londres, 1972.

[12] PALLU DE LA BARRIERRE, R. Quelques propriétes des multi-mesures, Seminaire d'analyse convexe, exposé no. 11, Montepellier, 1973.

[13] PALLU DE LA BARRIERRE, R. Multimesures à valeurs convexes fermées, Colloque sur l'intégration vectorielle et multivoque, exposé no. 4, Caen, 1975.

[14] PAPAGEORGIOU, N.S., Radon-Nikorlym theorems for multimeasures and transition multimeasures, Proc. Am. Math. Soc, n.2,pp +65-474,1991. 


\section{Índice Remissivo}

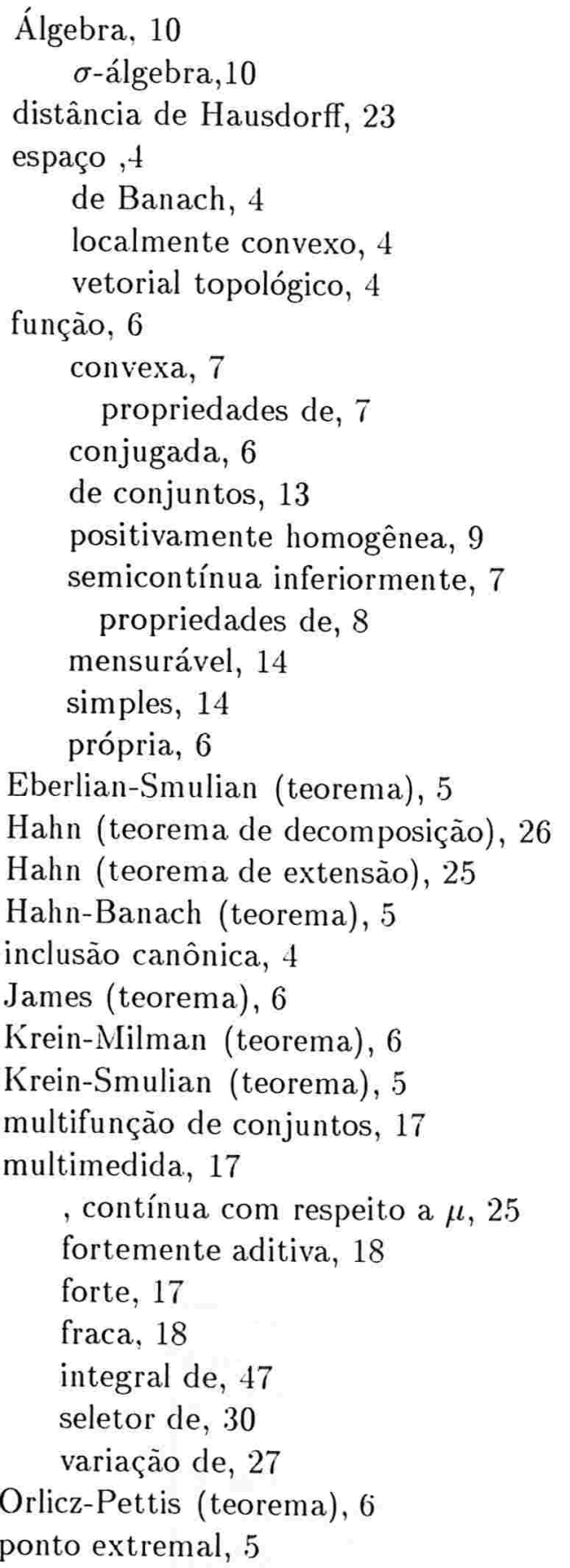

Princípio da limitação uniforme, 27

topologia fraca, 5

fraca-estrela, 5

Zermelo (teorema da boa ordem), 31

Zorn (lema de), 33 